\title{
Accelerator Physics at LEP
}

\author{
D.Brandt, H.Burkhardt, M.Lamont, S. Myers and J.Wenninger
}

\begin{abstract}
Accelerator physics issues and their influence on performance are presented for the Large Electron Positron storage ring (LEP) at CERN in Geneva, Switzerland. After several years of operation on the $\mathrm{Z}$ boson resonance at beam energies around $45 \mathrm{GeV}$, the beam energy was increased in steps to over $100 \mathrm{GeV}$. The major power loss to synchrotron radiation and its consequences on the maximum beam energy are discussed. The subjects of luminosity optimization, beam-beam effect, instabilities, detector backgrounds and beam lifetime are addressed. The precise beam energy calibration, which is of particular importance for the determination of standard model parameters is described.
\end{abstract}

Published in Rep. Prog. Phys. 63 (2000) 939-1000

Geneva, Switzerland

July 19, 2000 


\section{Contents}

1 Introduction $\quad 5$

2 LEP machine overview $\quad 6$

2.1 The LEP Injectors . . . . . . . . . . . . . . . . . . . . . . . . . . . . . 7

2.2 The LEP Operation Cycle . . . . . . . . . . . . . . . . . . . . . . . 8

3 Maximum energy 8

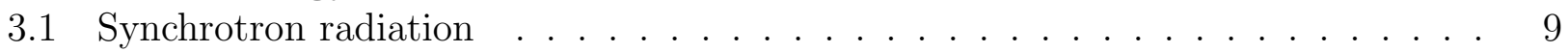

3.2 The beam energy . . . . . . . . . . . . . . . . . . . . . . . . . . 9

3.2.1 Active length of cavities . . . . . . . . . . . . . . . . . . . 10

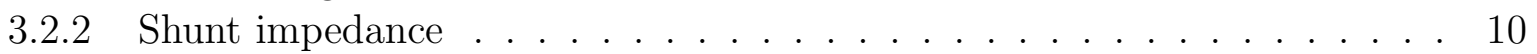

3.2.3 The over-voltage factor . . . . . . . . . . . . . . . . . . 11

3.2.4 The bending radius of the dipole magnets . . . . . . . . . . . . . . . 12

3.2.5 Contribution of smaller effects . . . . . . . . . . . . . . . . . 12

4 Luminosity 13

4.1 The number of bunches . . . . . . . . . . . . . . . . . . . . . . . 14

4.1.1 The Pretzel scheme . . . . . . . . . . . . . . . . . . . 15

4.1.2 The bunch train scheme . . . . . . . . . . . . . . . . . 15

5 Optics considerations $\quad 15$

5.1 Focusing strength . . . . . . . . . . . . . . . . . . 15

5.2 Choice of the optics . . . . . . . . . . . . . . . . . . . 17

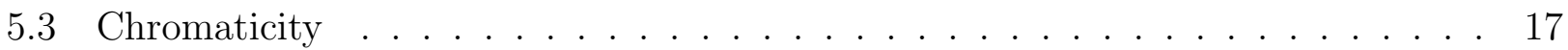

5.3.1 Momentum acceptance . . . . . . . . . . . . . . . 18

5.3 .2 Detuning with amplitude . . . . . . . . . . . . . . . . . . . . . . . . . . . 18

5.4 Minimum $\beta$ values at the interaction points . . . . . . . . . . . . . . 19

6 Radiation Damping and Beam Sizes $\quad 19$

6.1 Damping times and damping partition numbers . . . . . . . . . . . . . . . 20

6.2 Bunch length . . . . . . . . . . . . . . . . . . . . 20

6.3 Horizontal emittance . . . . . . . . . . . . . . . . . . . . . . 20

6.4 Vertical emittance . . . . . . . . . . . . . . . . . . . . . . 21

6.5 Variation of the damping partition numbers . . . . . . . . . . . . . . 22

7 Resonances 22

7.1 Resonance diagram . . . . . . . . . . . . . . . . . . . . . . . . . 23

7.2 Synchro-betatron resonances . . . . . . . . . . . . . . . . . . . 24

8 Collective effects and impedances 25

8.1 The loss factors . . . . . . . . . . . . . . . . . . . 25

8.2 Parasitic mode losses . . . . . . . . . . . . . . . . . . . . . 27

8.3 Head-tail instability . . . . . . . . . . . . . . . . . . . . . . . . . . . . . . . . . . . . . . 27

8.4 Transverse mode coupling instability . . . . . . . . . . . . . . . . . . . . . . . . . . . . . . . . 28

8.5 Coupled bunch instability . . . . . . . . . . . . . . . . . . . . . . . . 29 
9 Beam-beam interaction and limits 30

9.1 Beam-beam parameters . . . . . . . . . . . . . . . . . 30

9.2 Luminosity and beam-beam limits . . . . . . . . . . . . . . . . . . . . . . . . . . . . . . . . . . . . . . . 32

9.3 Beam-beam effects at LEP1 . . . . . . . . . . . . . . . . . . . . . . . . . . . . . . . . . . . . . . . . . . . . . . . . .

9.4 Beam-beam effects at LEP1.5 . . . . . . . . . . . . . . . . . . . . 35

9.5 Beam-beam effects at LEP2 . . . . . . . . . . . . . . . . . . . 35

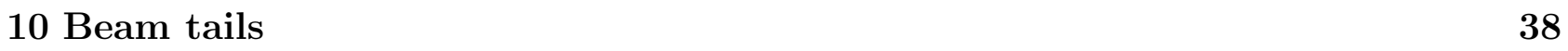

11 Beam lifetime $\quad 39$

11.1 Beam gas scattering . . . . . . . . . . . . . . . . . . . . . 40

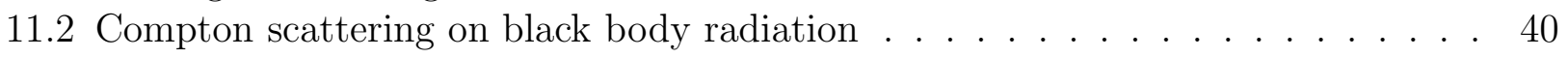

11.3 Radiative Bhabha scattering . . . . . . . . . . . . . . . . . . . . . 41

11.4 Lifetime observations at LEP . . . . . . . . . . . . . . . . . . . . . . 41

12 Background sources and shielding 43

12.1 Synchrotron radiation background . . . . . . . . . . . . . . . . . . 43

12.2 Off-momentum background . . . . . . . . . . . . . . . . . . . 44

13 Luminosity optimization 45

13.1 Head-on collisions . . . . . . . . . . . . . . . . . . . . . . . . . . . . . . . . . . . 45

13.1.1 Luminosity scans . . . . . . . . . . . . . . . . . 46

13.1.2 Beam-beam scans . . . . . . . . . . . . . . . . . . . . 46

13.2 Optical parameters at the interaction points . . . . . . . . . . . . . . . . . . . . . . . . . . . . 46

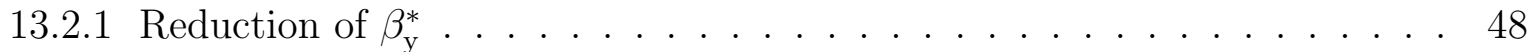

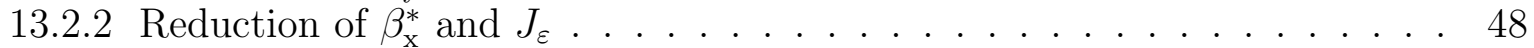

13.3 Beam emittance . . . . . . . . . . . . . . . . . . . . . . . . 49

13.3.1 Finite emission angle of synchrotron radiation . . . . . . . . . . . . 49

13.3.2 Dispersion at the IP . . . . . . . . . . . . . . . . . . . . 50

13.3.3 Coupling . . . . . . . . . . . . . . . . . 50

13.3.4 Residual vertical dispersion . . . . . . . . . . . . . . . . 50

13.3.5 Dispersion free steering . . . . . . . . . . . . . . . . . . 51

13.4 Luminosity equalisation . . . . . . . . . . . . . . . . . . . 52

13.4.1 Measurement and correction of the betatron functions . . . . . . . . . . 52

13.4 .2 Waist scans . . . . . . . . . . . . . . . . . . 52

13.4.3 Dispersion at the collision points . . . . . . . . . . . . . 52

13.4.4 Betatron function perturbations due to beam-beam effects . . . . . . . 53

13.4.5 Beams crossing at an angle . . . . . . . . . . . . . . 53

14 Energy calibration

14.1 Transverse polarization . . . . . . . . . . . . . . . . . . 54

14.2 Energy calibration by resonant depolarization . . . . . . . . . . . . . . . 55

$14.3 \mathrm{Z}$ boson resonance scans . . . . . . . . . . . . . . . . . . . . . . 56

14.3.1 Magnetic field stability . . . . . . . . . . . . . . . . . . . . . . . . . . . . . 58

14.3.2 Circumference variations and tides . . . . . . . . . . . . . . . . . . . . . . . . . . . . . . . 68

14.3.3 Centre-of-mass energies . . . . . . . . . . . . . . . . . . 60

14.3.4 Summary of LEP1 energy calibration . . . . . . . . . . . . . . . . . 62

14.4 Energy calibration at LEP2 . . . . . . . . . . . . . . . . . . . . . . . . . . . . . . . . . . . . . . . 62

14.4.1 The LEP spectrometer . . . . . . . . . . . . . . . . . . . . . . . . 62 
16 Appendix and symbols 


\section{Introduction}

Over the past decades electron positron colliders have been ideal tools for studying mesons $(J / \psi, \Upsilon)$ and leptons $(\tau)$. Although the actual discoveries have often occurred at proton machines, the precise and easily tunable beam energy, as well as the well defined initial state, are big assets of electron positron colliders. A great advantage of colliders in comparison to fixed target machines is the enormous gain in energy that one obtains in the centre-of-mass system. In a collider there is no distinction between beam and target, with each beam having about the same particle density. The density of a conventional target is, however, much higher and to have comparable event rates the beams in colliders are stored for many hours providing collisions over an extended period.

Following the prediction of the existence of two massive vector bosons, the neutral $\mathrm{Z}^{0}$ and the charged $\mathrm{W}^{ \pm}$, LEP was designed with the aim of discovering and studying those bosons, which were, however, first observed at the SPS proton-antiproton collider in 1982. With LEP it was possible to measure the properties of these bosons with excellent precision. A very important early result was that there are three types of light neutrinos and thus three fundamental fermion families. The precise determination of the standard model parameters from the LEP data allowed a prediction of the top mass and limits on the expected mass range for the standard Higgs boson [1].

Design studies of the LEP machine started at CERN in 1976 [2] and the first practical design was published in 1978 [3]. The proposed machine had a cost-optimized energy of $70 \mathrm{GeV}$ per beam and measured $22 \mathrm{~km}$ in circumference. After extensive discussions during the autumn of 1978 it was decided to embark on the design of a somewhat larger machine, $30 \mathrm{~km}$ in circumference, with a cost-optimized energy of about $90 \mathrm{GeV}$ per beam. The energy of both these machines could be extended by using super-conducting RF cavities, were these to become available, to $100 \mathrm{GeV}$ and above.

Studies of the $30 \mathrm{~km}$ machine were completed during 1979 and a design report was issued in August of that year [4]. These studies covered not only machine design but also the design and development of the components of LEP. A much cheaper design for the main magnet system was developed, as well as a more economical system for the RF accelerating system using a storage cavity scheme. At the same time, it was decided to increase the effort on the development of super-conducting RF cavities for LEP by setting up a small team at CERN and establishing a collaboration with other European laboratories. The basic feature of the final design was a machine with a large circumference which could be installed in stages to match the particle physics requirements and new technological developments [5].

This report is organized in the following way. Section 2 begins with a short overview of the LEP machine and of the CERN accelerator complex. The impact of the large energy loss to synchrotron radiation on the design of LEP and on its maximum energy is explained in section 3. The design considerations for luminosity, presented in section 4, depend largely on the beam intensities and on the beam spot sizes. While sections 5 and 6 describe how to reduce the beam sizes, sections 7 and 8 address limitations on the beam intensities due to resonances and collective effects of the particles in the beam. The mutual interaction of the beams at the collision points is a fundamental limit to accelerators operated as colliders. The impact of this "beam-beam" effect on the LEP performance is discussed in section 9. Problems related to beam tails, beam lifetimes and particle backgrounds in the detectors are addressed in sections 10 to 12. The daily optimization of the luminosity by the operations crews is explained in section 13 . The precise energy calibration of the beams and the dependence of the LEP energy on Earth tides, railway trains and geological movements are described in section 14. Finally a summary of the LEP performance is given in section 15. A glossary of frequently used symbols is included 
in the Appendix.

\section{LEP machine overview}

LEP produced its first collisions on August $13^{\text {th }}$ 1989, less than six years after ground was broken on September $13^{\text {th }}$ 1983. The construction of LEP was the largest civil engineering undertaking in Europe between 1983 and 1988. The ring extends from the foothills of the Jura mountain to the Geneva airport and straddles the border between France and Switzerland. The $3.8 \mathrm{~m}$ diameter machine tunnel is buried at a depth varying between 50 and $175 \mathrm{~m}$. It can be accessed through shafts at 8 access points numbered clockwise from 1 to 8 as shown in Figure 1. In the even numbered access points four large halls house the detectors of the four LEP experimental collaborations L3, ALEPH, OPAL and DELPHI. The beams are injected from the SPS ring on either side of point 1.

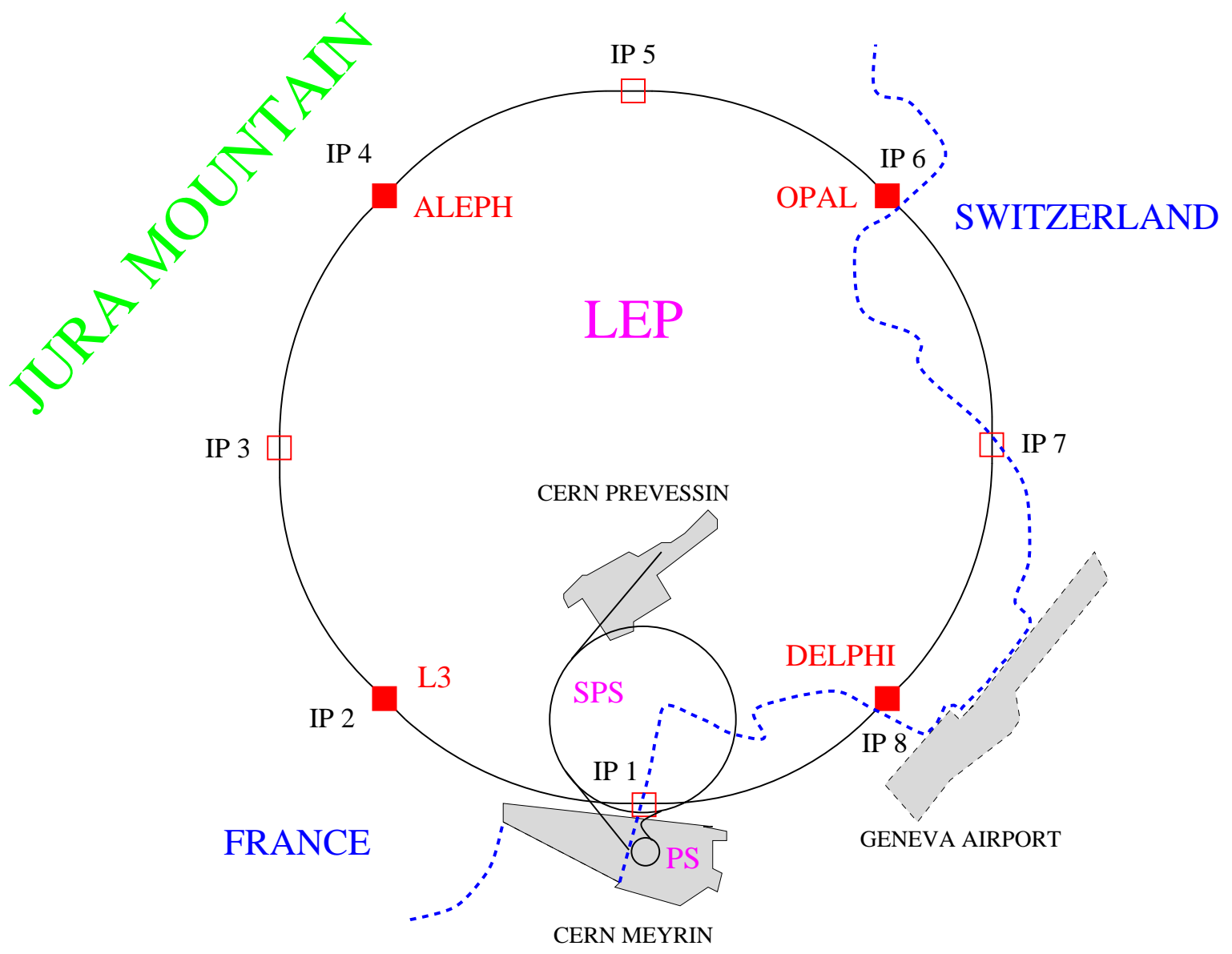

Figure 1: Layout of the LEP ring on the border of France and Switzerland. The 8 access points are denoted IP1 through IP8. The 4 LEP experiments L3, ALEPH, OPAL and DELPHI are installed at the even numbered access points. Positrons travel clockwise, electrons anticlockwise from points 1 to 8. The location of two LEP injectors, the SPS (Super Proton Synchrotron) and the PS (Protron Synchrotron), is also indicated.

The $26.65 \mathrm{~km}$ circumference LEP ring is composed of eight $2.9 \mathrm{~km}$ long arcs and eight straight sections extending for $210 \mathrm{~m}$ on either side of the 8 collision points. Some geometric parameters are given in Table 1. About 3400 dipole, 800 quadrupole, 500 sextupole and over 
600 orbit corrector magnets are installed in the tunnel. The magnet lattice is of FODO type with a period (cell) length of $79 \mathrm{~m}$ and 31 regular lattice periods per octant. The bending angle per period is $22.62 \mathrm{mrad}$. The radio-frequency acceleration system is installed in the straight sections around the 4 experiments. It is the largest $\mathrm{RF}$ system in the world with $288 \times 1.7 \mathrm{~m}$ long superconducting cavities and $48 \times 2.1 \mathrm{~m}$ long copper cavities providing more than $3.5 \mathrm{GV}$ of accelerating voltage [6]. A detailed specification of LEP can be found in [5].

Table 1: Geometric parameters of LEP. An extensive list of symbols used in the text can be found in the Appendix.

\begin{tabular}{lcc}
\hline Parameter & Symbol & Value \\
\hline Effective bending radius & $\rho$ & $3026.42 \mathrm{~m}$ \\
Revolution frequency & $f_{\text {rev }}$ & $11245.5 \mathrm{~Hz}$ \\
Length of circumference, $L=c / f_{\text {rev }}$ & $L$ & $26658.9 \mathrm{~m}$ \\
Geometric radius $(L / 2 \pi)$ & $R$ & $4242.9 \mathrm{~m}$ \\
Radio frequency harmonic number & $h$ & 31320 \\
Radio frequency of the $R F$-system, $f_{\mathrm{RF}}=h f_{\text {rev }}$ & $f_{\mathrm{RF}}$ & $352209188 \mathrm{~Hz}$ \\
\hline
\end{tabular}

\subsection{The LEP Injectors}

The first element of the LEP injector chain is a $200 \mathrm{MeV}$ electron linac. Its intense electron beam produces positrons in a tungsten target. Just downstream of the target a second linac accelerates positrons emerging from the target and electrons produced in a nearby gun to 600 $\mathrm{MeV}$. The linacs operate at $100 \mathrm{~Hz}$ and deliver beam pulses which are stored in eight bunches in the Electron-Positron Accumulator ring (EPA). The eight bunches are transfered to the CERN Proton Synchrotron (PS) where they are accelerated to $3.5 \mathrm{GeV}$. The final element in the chain is the CERN Super Proton Synchrotron (SPS) which delivers the beams to LEP at an energy of 20 or $22 \mathrm{GeV}$. The decision to use the already existing PS and SPS synchrotrons resulted in significant economies both in cost and time. To serve LEP the PS and SPS operate in multi-cycle mode whereby a sequence of proton and lepton acceleration cycles are repeated usually every 14.4 seconds. Up to $1.5 \times 10^{11}$ particles are delivered to LEP in each lepton cycle. Operation of the SPS as LEP injector has almost no effect on the $450 \mathrm{GeV}$ fixed-target proton beams. Table 2 and Figure 2 give an overview of the LEP injector chain which is described in $[5]$.

Table 2: Machine circumference (respectively length) $L$ and energy range for LEP and its injectors.

\begin{tabular}{lcccc}
\hline Accelerator & $L(\mathrm{~km})$ & $L / L_{\text {EPA }}$ & \multicolumn{2}{c}{ Energy $(\mathrm{GeV})$} \\
\cline { 3 - 5 } & & & Injection & Top \\
\hline Linacs & $\approx 0.1$ & - & 0 & 0.6 \\
EPA & 0.126 & 1 & 0.6 & 0.6 \\
PS & 0.628 & 5 & 0.6 & 3.5 \\
SPS & 6.912 & $5 \times 11$ & 3.5 & $20-22$ \\
LEP & 26.66 & $5 \times 11 \times 27 / 7$ & $20-22$ & 101 \\
\hline
\end{tabular}




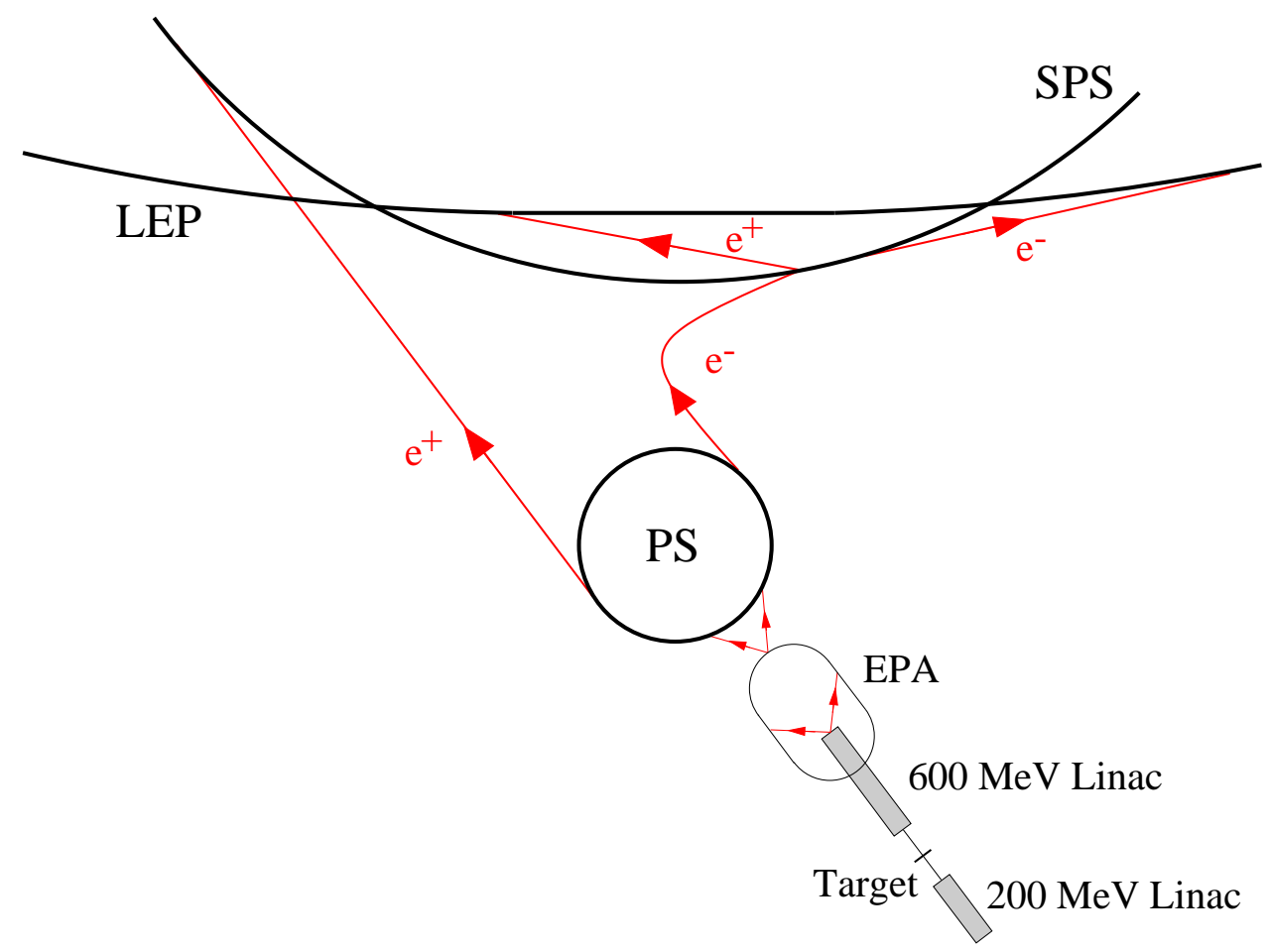

Figure 2: Layout of the LEP injector chain (dimensions not to scale).

\subsection{The LEP Operation Cycle}

Contrary to the fast cycling PS and SPS the LEP collider has a slow repetition rate. Each operation cycle, referred to as a "fill", begins by preparing the machine for injection at $22 \mathrm{GeV}$. Electrons and positrons are injected in parallel for typically 10 to 30 minutes depending on the desired beam intensities. During injection the beams are separated at all collision points using electrostatic separators. As soon as the filling process is ended the beams are accelerated to the target energy at a rate of $125 \mathrm{MeV} / \mathrm{s}$. During the ramp the beams sizes are also reduced at the collision points by a local change of the beam optics. When the beams have reached the target energy, conditions for physics data taking are set up in about 10 to 30 minutes. Various parameters like betatron tunes and beam orbits are adjusted, the beams are brought into collision and collimators are moved in place to protect the experiments against backgrounds. The LEP experiments start data taking as soon as the beam conditions are stable. The beams are typically kept in collision for 10 to 12 hours for beam energies around $45 \mathrm{GeV}$, and for 3 to 6 hours for energies above $90 \mathrm{GeV}$. The beams are then dumped by a fast kicker magnet and the operation cycle repeated. Typical efficiencies, defined as the ratio of the time spent taking data to the total time scheduled in advance for data taking, range between 40 and 60\% [7].

\section{Maximum energy}

The two fundamental parameters in the design of any particle collider are the beam energy range and the luminosity. The energy clearly defines the possible physics and the luminosity the possible event rate. In the case of LEP the energy was defined by the energy required to study the $\mathrm{Z}$ and the $\mathrm{W}^{ \pm}$particles and resulted in a range of 40 to $100 \mathrm{GeV}$ per beam. Having decided upon the energy range the next aim in the design procedure is to optimize the physical size of the collider so as to minimize the capital and running costs. For LEP, the crucial factor 
in the definition of the circumference is the problem of synchrotron radiation described in the following section.

\subsection{Synchrotron radiation}

The transverse acceleration produced in circular accelerators leads to the emission of electromagnetic radiation which is known as synchrotron radiation. This radiation is emitted in the forward direction tangential to the particle motion. For electrons, or positrons, the radiation power is given by:

$$
P_{\gamma}(G e V / s)=\frac{c}{2 \pi} \frac{c_{\gamma} E_{\mathrm{b}}^{4}}{\rho^{2}}
$$

where $c_{\gamma}$ is a constant of nature given by [8]

$$
c_{\gamma}=\frac{4 \pi}{3} \frac{r_{\mathrm{e}}}{\left(m_{\mathrm{e}} c^{2}\right)^{3}}=8.86 \times 10^{-5} \mathrm{~m} \mathrm{GeV}^{-3}
$$

It can be seen from Equation(1) that the amount of radiation is proportional to the fourth power of the particle energy and inversely proportional to the square of the bending radius in the dipoles $\rho$.

By integrating over the bending elements around the circumference of a circular accelerator, the energy loss per turn may be calculated :

$$
U_{0}=\frac{c_{\gamma} E_{\mathrm{b}}^{4}}{\rho}
$$

$U_{0}$ is the energy lost by each particle during a single revolution of the machine. Consequently, for a beam with $k_{\mathrm{b}}$ bunches of current $I_{\mathrm{b}}$, the total power loss to synchrotron radiation is :

$$
P_{\mathrm{b}}=\frac{U_{0} k_{\mathrm{b}} I_{\mathrm{b}}}{e}=\frac{E_{\mathrm{b}}^{4} k_{\mathrm{b}} I_{\mathrm{b}}}{e \rho} \times 8.86 \times 10^{-2}(\mathrm{MW})
$$

where the energy $E_{\mathrm{b}}$ is expressed in $\mathrm{GeV}$ and the bunch current $I_{\mathrm{b}}$ in Amperes. The strong dependence of the radiation on the particle energy sets severe practical limitations on the maximum achievable energy of a circular lepton collider. First of all, the energy lost has to be replenished by a RF system and secondly, the different hardware constituents of the collider have to be able to cope with power deposited by the synchrotron radiation. In LEP at $100 \mathrm{GeV}$, the radiated power for a total intensity of $6 \mathrm{~mA}$ is about $18 \mathrm{MW}$.

\subsection{The beam energy}

The RF acceleration system must replace the energy lost by the particles to synchrotron radiation, and the power and cost of the RF system is defined to a large extent by the need to meet this requirement.

The voltage in the accelerating gaps of the RF cavities oscillates at a high frequency $(352 \mathrm{MHz}$ for LEP) and is synchronised to a harmonic $(h)$ of the revolution frequency of the particles. Ideally the bunches traverse the accelerating gaps at a constant phase $\left(\phi_{\mathrm{s}}\right)$ relative to the voltage. In this way particles gain energy as they traverse the cavities. The average energy gain per turn must compensate the energy loss per turn due to synchrotron radiation i.e.

$$
U_{0}=e \hat{V} \sin \phi_{\mathrm{s}}
$$


where $\hat{V}$ is the peak accelerating RF voltage. In addition, power is dissipated as heat in the accelerating resonant $\mathrm{RF}$ cavity wall. The dissipated power $P_{\mathrm{d}}$ is given by:

$$
P_{\mathrm{d}}=\frac{\hat{V}^{2}}{l R_{\mathrm{s}}}
$$

where $l$ is the total active cavity length, and $R_{\mathrm{s}}$ the shunt impedance per metre of the accelerating structure.

Combining equations $(3),(6)$, and (5) gives the power dissipation in the cavity walls $\left(P_{\mathrm{d}}\right)$ as a function of the relevant beam parameters

$$
P_{\mathrm{d}}=\left(\frac{c_{\gamma}}{e}\right)^{2} \frac{E_{\mathrm{b}}^{8}}{l R_{\mathrm{s}} \rho^{2} \sin ^{2} \phi_{\mathrm{s}}}
$$

The power absorbed by the beams is

$$
P_{\mathrm{b}}=2 k_{\mathrm{b}} I_{\mathrm{b}} \frac{U_{0}}{e}=2 k_{\mathrm{b}} I_{\mathrm{b}} \frac{c_{\gamma} E_{\mathrm{b}}^{4}}{e \rho} .
$$

The total power needed then is simply

$$
P_{\text {total }}=P_{\mathrm{d}}+P_{\mathrm{b}}
$$

For the case of room temperature accelerating cavities, the power dissipated in the cavity walls is predominant and scales with the eighth power of the energy. For a given beam energy, this power may be minimized by optimizing

1. the total active length of the cavities, $l$;

2 . the shunt impedance per metre of each cavity, $R_{\mathrm{s}}$;

3. the "over-voltage factor", $q=1 / \sin \phi_{\mathrm{s}}$;

4. the bending radius of the bending magnets, $\rho$.

Each of these options is considered in turn below.

\subsubsection{Active length of cavit ies}

Clearly from equation (7), increasing the active cavity length will reduce the power dissipated in the cavity walls. However, besides the increased cost due to the increase of the number of cavities, this approach has two undesirable consequences:

1. The length of the RF straight sections which house the cavities would increase. This would necessitate an increase in the circumference of the tunnel as well as increased cost due to civil engineering and the need for additional equipment.

2. The total impedance seen by the beam increases linearly with the number of cavities. This impedance can limit the performance of the collider, as will be described later.

\subsubsection{Shunt impedance}

The effective shunt impedance of resonant cavities can be increased by optimization of the geometry of the cavities; in particular the beam port hole diameter has a strong influence. However, reduction of the beam port radius not only increases the shunt impedance but also increases the transverse impedance. In fact the transverse impedance increases with approximately the third power of this parameter. For this reason the beam port radius in the accelerating cavities was not pushed to the aperture limit of the LEP beams. The effective shunt impedance in LEP was also increased by the addition of a low loss "storage cavity" coupled to the accelerating cavity. This technique increases the effective shunt impedance by $\sim 55 \%$. 


\subsubsection{The over-voltage factor}

The RF voltage in the cavity gaps varies sinusoidally in time. An idealised on-energy, on-orbit particle will arrive with the nominal, synchronous phase on each passage through the cavities. Any energy variation with respect to the nominal translates into a variation in time of arrival at a cavity: in an ultra-relativistic machine like LEP where the velocity is practically independent of energy, any particles with more energy than nominal travel further and take longer on their circuit of the machine; the reverse is true of particles with less energy. The variation in time of arrival means that particles with more energy see less RF voltage and vice versa, and there is a restoring longitudinal force about the synchronous phase leading to harmonic oscillations about this point. Thus as a particle circulates it performs so-called synchrotron oscillations in energy and azimuthal position with respect to the synchronous particle circulating with nominal phase.

There is a maximum amplitude of stable synchrotron oscillations and the associated maximum energy deviation determines the energy acceptance of the machine. Particles with amplitudes above this will be lost.

The over-voltage factor (q) is the ratio between the peak RF voltage and the energy lost per turn to synchrotron radiation by the synchronous particle, and from equation (5) is equal to $\left(\sin \phi_{\mathrm{s}}\right)^{-1}$. The energy acceptance may be expressed in terms of this over-voltage factor:

$$
\left(\frac{\Delta E}{E}\right)=\sqrt{\frac{2 U_{0}}{\pi \alpha_{\mathrm{c}} h E_{\mathrm{b}}}\left(\sqrt{q^{2}-1}-\arccos \frac{1}{q}\right)} .
$$

The momentum compaction factor $\alpha_{\mathrm{c}}$ quantifies the variation of orbit length (and thus time of arrival at the cavities) with energy.

The energy oscillations are damped at a rate proportional to the change of radiation loss with energy. When a particle has an energy deviation $\epsilon>0$ with respect to the synchronous particle it radiates slightly more energy that it does when $\epsilon<0$. This causes the particle to spiral slowly inwards in phase space as it performs synchrotron oscillations, thus damping its motion.

The emission of synchrotron radiation is, however, a quantum process which takes place over a wide range of photon energies. The discrete nature of photon emission causes a particle to perform a random walk through synchrotron phase space. This is essentially a stochastic process and in the linear approximation the system can be viewed as being a thermal equilibrium between the damping and excitation effects. The energy spread of the beam is a balance between radiation damping and the excitations caused by fluctuations in the radiated energy about its mean rate. The energy spread in an iso-magnetic machine is given by [9] :

$$
\frac{\sigma_{\mathrm{e}}}{E_{\mathrm{b}}}=\gamma \sqrt{\frac{c_{\mathrm{q}}}{J_{\varepsilon} \rho}}
$$

where $\gamma$ is the Lorentz factor $\gamma=E_{\mathrm{b}} /\left(m_{\mathrm{e}} c^{2}\right), J_{\varepsilon}$ is the longitudinal damping partition number, see Section 6, and

$$
c_{\mathrm{q}}=\frac{55 \hbar c}{32 \sqrt{3} m_{\mathrm{e}} c^{2}}=3.832 \times 10^{-13} \mathrm{~m} .
$$

When energy spread of the particle distribution approaches the energy acceptance there is a non-zero probability that the energy fluctuations of some particles may cause them to "jump" outside the stable region. In practice this will result in those particles continuously losing energy until they are lost against the inner vacuum chamber wall. The half-life due to this effect, usually referred to as the quantum lifetime, is given by [8]:

$$
\tau_{\mathrm{q}}=\tau_{\varepsilon}\left(\frac{\sigma_{\mathrm{e}}}{\Delta E}\right)^{2} \exp \left(\frac{\Delta E^{2}}{2 \sigma_{\mathrm{e}}^{2}}\right) .
$$


Thus in evaluating the required RF voltage and stable phase angle one has to provide enough volts to replace the energy loss to synchrotron radiation and provide sufficient energy acceptance to maintain a reasonable quantum lifetime.

From equation (10), it can be seen that the energy acceptance is proportional to $(\sqrt{h})^{-1}$ at fixed RF voltage. Thus one possible means of minimizing the required $\mathrm{RF}$ voltage is to operate at the minimum possible RF frequency $\left(f_{\mathrm{RF}}=h f_{\mathrm{rev}}\right)$. However reducing the frequency of the accelerating structure is severely constrained for two main reasons. Firstly the physical size of the structures increases as does the cost. Secondly, high power RF sources, such as klystrons, are uneconomical at frequencies below around $300 \mathrm{MHz}$. For these reasons the frequency of the accelerating structure was chosen to be $352 \mathrm{MHz}$ which corresponds to 31320 times the revolution frequency.

\subsubsection{The bending radius of the dipole magnet $s$}

From equations (7) and (8), it is clear that the bending radius $\rho$ is a critical parameter in the minimization of the demanded RF power at a given energy. In the early design stage of the LEP collider it was considered essential that the energy needed for W-pair production could be reached by the use of conventional room temperature accelerating cavities and not be totally dependent on the future development of super-conducting structures. Nevertheless a huge effort was put into such development since it was known that great savings could result from the improvement in operational efficiency of the collider. At the time an upper limit on the amount of high frequency power was considered to be around $100 \mathrm{MW}$. This upper limit combined with the then known cavity gradients and shunt impedances gave, using equation (7), a bending radius of around $3.5 \mathrm{~km}$. This value and the length of the straight sections of more than $2 \mathrm{~km}$ in length needed to house the 768 normal conducting cavities brought the circumference of LEP to $27 \mathrm{~km}$. It may now be said that this optimum value for the bending radius would have been slightly less $(\sim 10 \%)$ if it had been optimized for super-conducting cavities. However this margin is already being used as LEP goes to energies beyond those previously foreseen.

For phase 1 of LEP, the energy required was only around $46 \mathrm{GeV}$ per beam, and much less RF power was required; in fact only $16 \mathrm{MW}$. Consequently it was decided for this initial phase to buy and install only one sixth of the foreseen room temperature cavities and continue with the development of super-conducting cavities. In this way both options were left open for the second phase. The final scheme for increasing the beam energy to beyond $100 \mathrm{GeV}$ required the installation of 288 super-conducting cavities with an additional required power of around $36 \mathrm{MW}$.

\subsubsection{Contribution of smaller effects}

The discussion presented in this chapter has been approximate in several respects. We only considered the main source of energy loss, the synchrotron radiation in the main dipoles and neglected local variations in beam energy and energy loss around the ring.

The energy loss per turn reaches about $3 \%$ of the beam energy at $100 \mathrm{GeV}$. The loss is compensated by the acceleration in the RF-system, concentrated approximately symmetrically in four points around the ring. There remains a variation in beam energy of up to $0.75 \%$ around the ring, often referred to as the energy sawtooth.

Due to finite beam sizes, energy sawtooth and orbit imperfections, there is also synchrotron radiation in quadrupoles, typically at a level of $0.2 \%$ compared to the radiation in the bending magnets[10]. In addition, there is also the parasitic energy loss (about $11 \mathrm{MeV} / \mathrm{turn}$ for 0.6 
$\mathrm{mA}$ bunch current [11]). On the other hand, the prediction for needs on RF-voltage for a given quantum lifetime decrease by about $2 \%$ when higher order corrections are taken into account [12]. The main effect can be understood qualitatively as follows: the quantum lifetime is due to particles which are lost from the RF-bucket. These particles do not have the nominal energy, but rather $1.4 \%$ less energy (in the case of LEP2). In consequence they radiate less synchrotron radiation and are more effectively focused by the RF-voltage.

\section{Luminosity}

In any collider, one of the most important performance parameter is the luminosity $(\mathcal{L})$ which defines the number of events $(N)$ produced per second at each interaction region

$$
\frac{d N}{d t}=\sigma \mathcal{L}
$$

where $\sigma$ is the cross section of the process.

For $\mathrm{e}^{+} \mathrm{e}^{-}$colliders the general equation for luminosity is

$$
\mathcal{L}=\frac{N_{\mathrm{b}+} N_{\mathrm{b}-} f_{\mathrm{rev}} k_{\mathrm{b}} \exp \left\{-\frac{\left(\overline{\mathrm{x}}_{-}-\overline{\mathrm{x}}_{+}\right)^{2}}{2\left(\sigma_{\mathrm{x}+}^{2}+\sigma_{\mathrm{x}-}^{2}\right)}-\frac{\left(\bar{y}_{-}-\bar{y}_{+}\right)^{2}}{2\left(\sigma_{\mathrm{y}+}^{2}+\sigma_{\mathrm{y}-}^{2}\right)}\right\}}{2 \pi \sqrt{\left(\sigma_{\mathrm{x}+}^{2}+\sigma_{\mathrm{x}-}^{2}\right)\left(\sigma_{\mathrm{y}+}^{2}+\sigma_{\mathrm{y}-}^{2}\right)}}
$$

where $N_{\mathrm{b}-}$ and $N_{\mathrm{b}+}$ are the number of electrons and positrons per bunch. $k_{\mathrm{b}}$ is the number of bunches per beam, $\left(\bar{x}_{-}-\bar{x}_{+}\right),\left(\bar{y}_{-}-\bar{y}_{+}\right)$are the horizontal and vertical distances between the centres of gravity of the electron and positron beams and $\sigma_{\mathrm{x}-}, \sigma_{\mathrm{x}+}, \sigma_{\mathrm{y}-}$, and $\sigma_{\mathrm{y}+}$ are the r.m.s. horizontal and vertical beam dimensions of the electron and positron beams given by $(\mathrm{z}$ $=\mathrm{x}, \mathrm{y})$ :

$$
\sigma_{\mathrm{z}}=\sqrt{\sigma_{\beta, \mathrm{z}}^{2}+\sigma_{\mathrm{e}}^{2}}=\sqrt{\beta_{\mathrm{z}} \epsilon_{\mathrm{z}}+\left(D_{\mathrm{z}} \frac{\sigma_{\mathrm{e}}}{E_{\mathrm{b}}}\right)^{2}}
$$

where $\sigma_{\beta, \mathrm{z}}$ is the beam size coming from betatron (transverse) motion and $\sigma_{\mathrm{e}}$ is the beam size coming from the energy spread. The betatron function $\beta_{\mathrm{z}}$ is determined by the beam optics and defines the local beam envelope. $\epsilon_{\mathrm{z}}$ is the betatron emittance, i.e. the phase space volume occupied by the beam. The dispersion $D_{\mathrm{z}}$ is defined by

$$
D_{\mathrm{z}}=\frac{\Delta z}{\Delta p_{\mathrm{b}} / p_{\mathrm{b}}}
$$

and measures the local sensitivity of the beam position to changes of the beam momentum $p_{\mathrm{b}}$. In general the vertical dispersion $D_{\mathrm{y}}$ is much smaller than the horizontal dispersion $D_{\mathrm{x}} \cdot \sigma_{\mathrm{e}} / E_{\mathrm{b}}$ is the relative energy spread (more accurately the relative momentum spread).

A fundamental limitation to all electron-positron colliders results from the influence of the electro-magnetic field associated with each beam on the motion of the particles in the "other" beam. In the case of head-on collisions this "beam-beam effect" is quantified by the beam-beam strength parameter $(\xi)$. For the vertical plane

$$
\xi_{\mathrm{y}}=-\frac{\beta_{\mathrm{y}}^{*}}{4 \pi} \frac{\Delta y^{\prime}}{y_{0}}
$$

where $\Delta y^{\prime}$ is the angular deflection $\left(\Delta \frac{d y}{d s}\right)$ received by a particle at a vanishing displacement from the centre of the other beam $y_{0}, \mathrm{~s}$ is the longitudinal coordinate, and $\beta_{\mathrm{y}}^{*}$ is the betatron 
amplitude function at the location of the beam-beam interaction. By convention the subscript $(*)$ refers to the $\beta$ function at the interaction points.

Evaluation of the deflection $\Delta y^{\prime}$ requires calculation of the electrical potential of the threedimensional Gaussian charge distribution of the beam. For small displacements, and for $\sigma_{\mathrm{x}} \gg$ $\sigma_{\mathrm{y}}$, Equation (18) becomes

$$
\xi_{\mathrm{y}}=\frac{N_{\mathrm{b}} r_{\mathrm{e}}}{2 \pi \gamma} \frac{\beta_{\mathrm{y}}^{*}}{\sigma_{\mathrm{x}} \sigma_{\mathrm{y}}} .
$$

Combining Equations (15) and (19) and assuming that the bunches in both beams have the same number of particles $\left(N_{\mathrm{b}}\right)$ and the same sizes $\left(\sigma_{\mathrm{x}}, \sigma_{\mathrm{y}}\right)$ gives the luminosity in a more useful form, i.e.

$$
\mathcal{L}=\frac{\gamma f_{\mathrm{rev}} k_{\mathrm{b}}}{2 r_{\mathrm{e}}} \frac{N_{\mathrm{b}} \xi_{\mathrm{y}}}{\beta_{\mathrm{y}}^{*}}
$$

\subsection{The number of bunches}

From Equation (20), it is clear that the luminosity may be increased by increasing the number of bunches. For a collider with $n_{\mathrm{c}}$ experimental collision points, the minimum number of bunches required to provide collisions in all experiments is $n_{\mathrm{c}} / 2$. There are several consequences associated with increasing the number of bunches beyond this minimum value.

- The Parasitic Beam-Beam Effect: It is undesirable to allow the bunches to interact in the unwanted collision points for two main reasons. Firstly the ability to maximise the beam-beam strength parameter is reduced because of the contributions from the additional collisions, and secondly the lifetime of the beam intensity is reduced due to the increased number of collisions. The beams may be separated in the unwanted collision points by the use of electro-static separators. These generate a linear electric field which deflects the electrons in one direction and the positrons in the other. By the use of sets of such separators closed bumps can be created which cause the bunches to pass each other with a vertical displacement.

- Beam Power: Increasing the number of bunches increases the total beam current and hence the required RF power (see Equation (8)). The beam power for LEP phase 1 is only 1.2 MW, however for phase 2 the beam power is up to $18 \mathrm{MW}$ for the design current of $3 \mathrm{~mA}$ per beam. Consequently increasing the number of bunches by say a factor of ten would necessitate an unacceptably high beam power.

- Detector Considerations: The electronics in experimental detectors have finite times available for particle identification and triggering sequences. The complexity and cost of these electronics increases as the time between successive collisions decreases (or the number of bunches increases).

The original LEP design assumed that the electron and positron beams each consist of four equidistant bunches. These collide in the four interaction regions where an experiment is installed and are vertically separated by electrostatic separator bumps in the interleaved interaction points which are not equipped with an experiment. LEP was operated in this manner from 1989 to 1992. Because of a limitation in the bunch current, the most promising way of further increasing the luminosity was by increasing the number of bunches in each beam. Two approaches have been successfully tested in LEP, namely the Pretzel scheme and the Bunch Trains scheme. 


\subsubsection{The Pretzel scheme}

The idea for the Pretzel scheme originated at the Cornell Electron Storage ring CESR, where the scheme has been used since 1983 [13]. In this scheme, the electrons and positrons travel on orbits which are distorted in opposite directions over practically the whole circumference of the machine by horizontal electrostatic fields. Parasitic beam-beam collisions can be arranged such that they occur where the separation between the two beams is almost maximum. Such a scheme has a potential for allowing a large number of bunches per beam. However, in the case of LEP, hardware and cost optimizations resulted in a scheme with 8 equidistant bunches per beam [14]. The sketch of the Pretzel scheme used in LEP from 1992 to 1994 is illustrated in Figure 3.

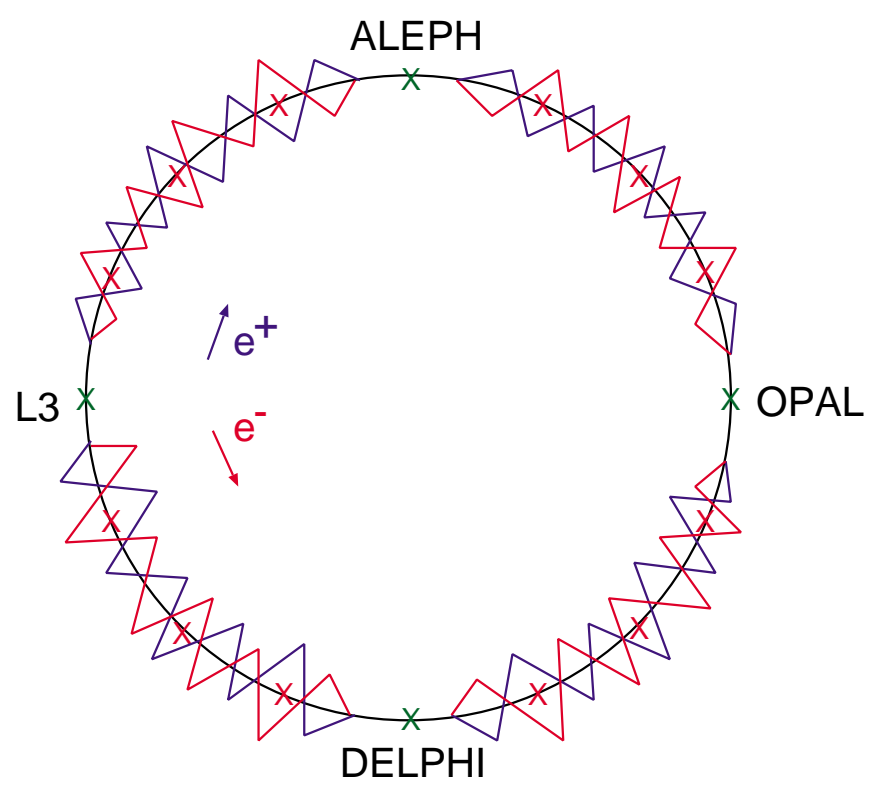

Figure 3: Schematic layout of the Pretzel scheme used in LEP. The beams are separated horizontally in the mid-arcs to avoid unwanted collisions.

\subsubsection{The bunch train scheme}

The principle of the bunch train scheme is to string together individual bunches in trains wherein the distance between the bunches of a train is much smaller than the distance between the trains. In such a scheme, separation bumps are used to maximise the distance between bunches at potential parasitic encounters. The basic principle of the scheme is illustrated in the Figure 4 where the case of three bunches per train is shown. The bunch train scheme has been successfully used in LEP since 1995 with initially three and finally two bunches per train and four trains in each beam (e.g. a total of 16 bunches in the machine). A detailed description of the scheme is presented in Reference [15].

\section{Optics considerations}

\subsection{Focusing strength}

From the formula for luminosity Equation(15) it is clear that the performance of the machine depends on the beam sizes at the interaction points $\sigma_{\mathrm{z}}(\mathrm{z}=\mathrm{x}, \mathrm{y})$, where, with vanishing 


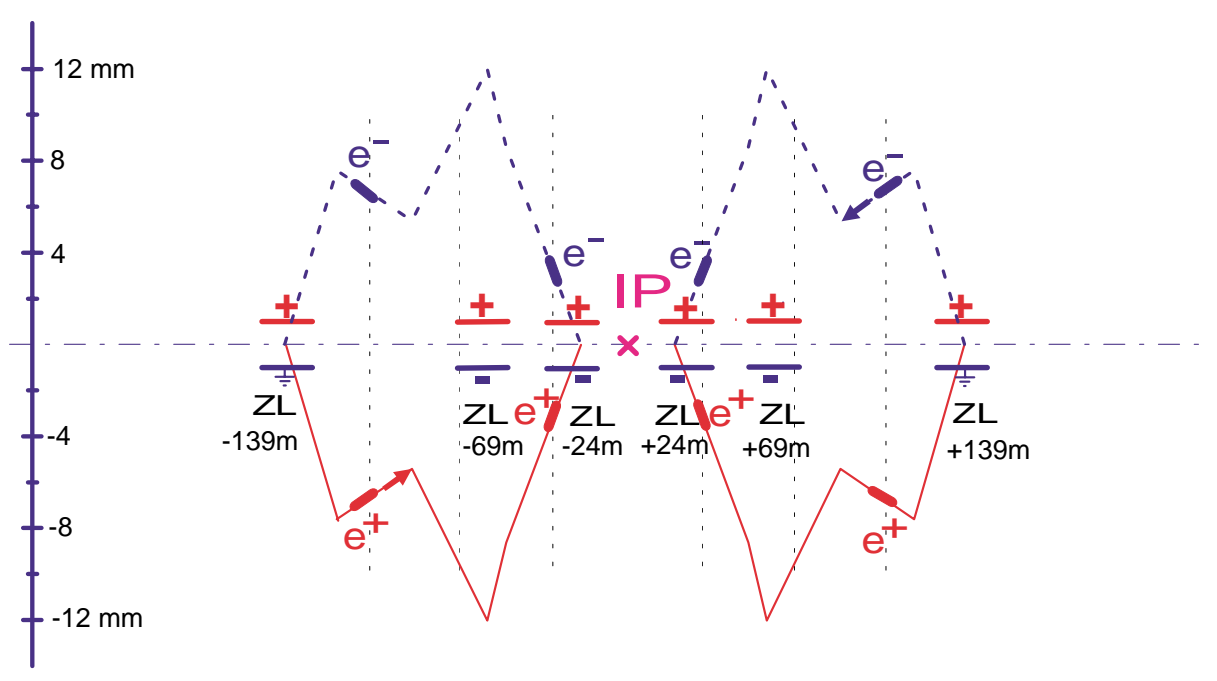

Figure 4: Schematic layout of the Bunch Trains scheme used in LEP. The beams are separated vertically around the eight interaction regions. The dotted vertical lines indicate the longitudinal position of parasitic encounters. Six electrostatic separators (ZL) are used to build the vertical separation bump.

dispersion,

$$
\sigma_{\mathrm{z}}=\sqrt{\beta_{\mathrm{z}}^{*} \epsilon_{\mathrm{z}}} .
$$

Consequently the beam size at the interaction point can be reduced by lowering the value of the $\beta$ function $\left(\beta_{\mathrm{z}}^{*}\right)$, i.e. the beam envelope. This can be done by varying the strength of the quadrupoles in the interaction regions. The minimum value of $\beta_{\mathrm{z}}^{*}$ will depend on the available strength of these quadrupoles. It is worth underlining that the quadrupole parameters are defined in the design phase of the project and this initial choice could limit further developments during the subsequent operation of the machine.

The focusing strength of a quadrupole $K$ is defined:

$$
K\left(\mathrm{~m}^{-2}\right)=\frac{G(\mathrm{~T} / \mathrm{m})}{3.3356 \times p_{\mathrm{b}}(\mathrm{GeV} / \mathrm{c})}
$$

where $G$ is the gradient $\partial B_{\mathrm{x}} / \partial y$ and $p_{\mathrm{b}}$ the beam momentum. Reduction of the beam size either by a stronger focusing in the arcs or by stronger local focusing near an interaction point necessarily implies larger values of $K$. However, for a given gradient, this will automatically impose an upper limit on the maximum energy at which the machine can be operated.

In LEP there exist essentially three different types of quadrupoles. Those most numerous are distributed along the arcs of the machine and have a gradient of $9.5 \mathrm{~T} / \mathrm{m}$. Some dedicated quadrupoles in the straight sections of the machine have a gradient of $11 \mathrm{~T} / \mathrm{m}$ and special super-conducting quadrupoles which are located close to the interaction points have a gradient of $55 \mathrm{~T} / \mathrm{m}$. This latter type are used for minimizing the vertical beam size at the interaction points.

In its present configuration, the lattice properties of LEP have been chosen such that an operation up to $105 \mathrm{GeV}$ should be possible without reaching a limit on the available strength 
of the different quadrupoles installed in the machine.

\subsection{Choice of the optics}

In a circular accelerator the bending of the beam is performed by dipole magnets. Quadrupole magnets provide focusing that keep the particles moving around a stable closed orbit. As a particle moves around the ring it performs transverse, so-called betatron, oscillations around this orbit. The number of betatron oscillations a particle performs during one revolution around the ring is called the betatron tune $Q$. For LEP $Q$ varies between 60 and 100 depending on the beam optics.

Typically in the arcs of an accelerator like LEP one has a regular, periodic structure, consisting of interleaved quadrupole and bending magnets. The structure that is repeated is called a cell and the most typical is the FODO cell consisting of: focusing quadrupole, bending magnet, de-focusing quadrupole, bending magnets etc.

The term "optics" can be used generally to refer to the overall choice of magnet element strengths or more specifically the choice of the quadrupole strengths in the periodic structure of the arcs. The latter is labelled by the fraction of a complete betatron oscillation which is performed per cell. For example, the first optics used in LEP was a $60^{\circ} / 60^{\circ}$ optics. The first number indicates the phase advance per cell in the horizontal plane, while the second number gives the corresponding value for the vertical plane. A large phase advance is, in general, indicative of stronger quadrupole strengths and smaller emittances, see Equation (30). During its lifespan, LEP has been successfully operated with $60^{\circ} / 60^{\circ}, 90^{\circ} / 60^{\circ}, 90^{\circ} / 90^{\circ}$ and $102^{\circ} / 90^{\circ}$ optics.

The choice of phase advance per cell has been a key one in LEP's move to higher energy. The horizontal beam size naturally increases with energy and stronger focusing in the horizontal plane is required to keep the horizontal beam size within manageable limits and to maintain the luminosity performance. The strong focusing optics have the additional advantage of a smaller momentum compaction factor allowing a slightly higher energy to be achieved for a given RF voltage, here illustrated in Figure 5 for two different LEP optics.

Unfortunately, such stronger focusing has a drawback, namely the associated increase in the sextupole strengths required for chromatic correction. Such strongly excited sextupoles can modify the non-linear dynamics of the machine and may eventually result in an intolerable reduction of the available dynamic aperture. The ultimate choice of the optics will therefore result from a trade off between considerations of hardware, beam dynamics, performance and physics objectives.

\subsection{Chromaticity}

The focusing properties of a quadrupole are momentum dependent, in that high (low) energy particles are focused less (more) than nominal energy particles. The tune of a particle will therefore vary with momentum. This variation of tune with momentum $p_{\mathrm{b}}$ defines the chromaticity $Q^{\prime}:$

$$
Q^{\prime}=\frac{\Delta Q}{\Delta p_{\mathrm{b}} / p_{\mathrm{b}}}
$$

Chromatic effects can be quite large and have severe implications for the stability of particle motion. It is therefore extremely important to compensate this "natural" chromaticity.

The chromatic compensation is performed with distributed sets of sextupole magnets located at positions where the dispersion function is large. In this way, particles with momentum 


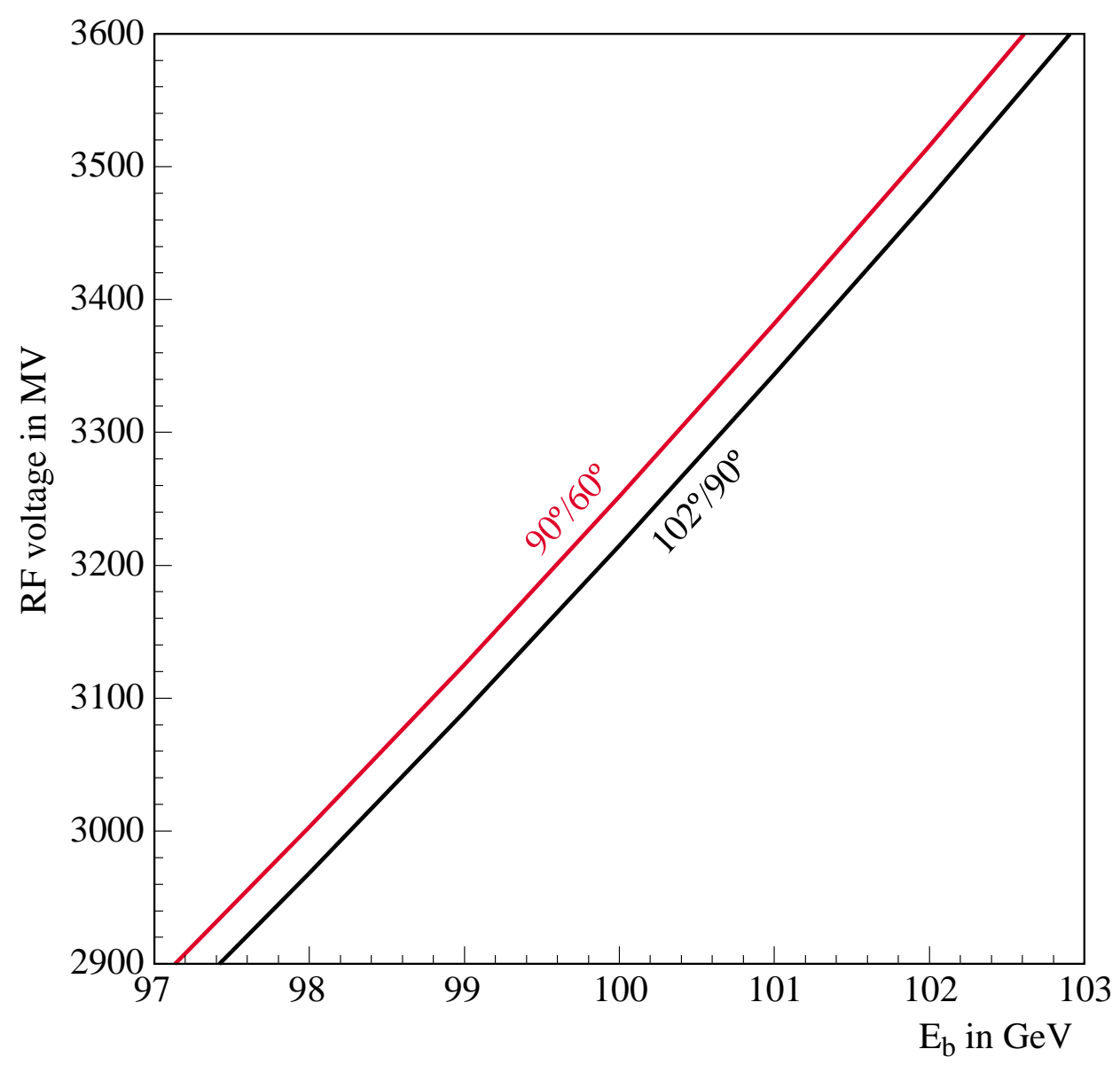

Figure 5: Maximum energy as a function of the available RF voltage for different LEP optics. The curves have been calculated for a quantum lifetime of 100 hours.

deviations pass through the sextupoles with a transverse offset and so see a different compensating quadrupolar field. The basic principles of the non-linear chromaticity correction are thoroughly described in Reference[16].

\subsubsection{M oment um acceptance}

The sextupoles are grouped and powered in families (with typically 3 families in the horizontal and 2 in the vertical plane). One criterion optimized by differential powering of these families is the detuning as a function of momentum. As a result, there is a range of momenta for which the tunes of the particles remain within an acceptable range. This range is called the momentum acceptance of the machine. A momentum acceptance of about $\pm 2 \%$ was measured at injection by recording the accumulation efficiency as a function of the energy of the injected beam. The physics optics has a smaller $\beta_{\mathrm{y}}^{*}$ and a reduced momentum acceptance. It should be at least $\pm 1 \%$ and not be less than the energy acceptance of Equation (10) necessary for a good beam (quantum) lifetime.

\subsubsection{Det uning with ampl it ude}

The sextupoles strongly modify the tune of a particle as a function of the betatron amplitude. This effect can be described by four terms: vertical detuning w.r.t. vertical amplitude, horizontal detuning w.r.t. horizontal amplitude and the two cross-terms, which have to be evaluated 
for each sextupole compensation scheme. In practice, these terms can reach very large values, and, as a consequence, the tune of particles with relatively low amplitude can shift onto resonances. In LEP, the large detuning with amplitude of some optics produced strong nonGaussian tails in the transverse beam distributions, which reduced the lifetime and eventually prevented effective operation of the machine.

\subsection{Minimum $\beta$ values at the interaction points}

The variation of the $\beta$ function as function of the drift distance $s$ from the interaction point is given by :

$$
\beta_{\mathrm{y}}(s)=\beta_{\mathrm{y}}^{*}\left(1+\frac{s^{2}}{\left(\beta_{\mathrm{y}}^{*}\right)^{2}}\right) \simeq \frac{s^{2}}{\beta_{\mathrm{y}}^{*}} \quad \text { for } \quad s \gg \beta_{\mathrm{y}}^{*} .
$$

Consequently, reduction of the $\beta$ value at a collision point can result in very large $\beta$ values at the nearest quadrupoles. There exists therefore a minimum $\beta^{*}$ which results from the physical aperture constraints in these quadrupoles. In general, however, the minimum value of $\beta$ is limited by other effects.

For example, beam-beam simulations [17] have shown that $\beta_{\mathrm{y}}^{*}$ should be at least twice the r.m.s. bunch length. In LEP at high energy this implies $\beta_{\mathrm{y}}^{*} \geq 2 \mathrm{~cm}$. In practice, such a low value is ruled out: the high strength required in the nearest quadrupoles would generate excessive chromaticity.

The lowest value achieved so far in operation is $\beta_{\mathrm{y}}^{*}=4 \mathrm{~cm}$. Furthermore, it should be remembered that large values of the sextupole strength strongly enhance the excitation of non-linear resonances and increase the tune dependence on amplitude. Both effects are known to seriously affect the dynamic aperture.

In the horizontal plane the minimum value of $\beta_{\mathrm{x}}^{*}$ is limited by background considerations : the choice of $\beta_{\mathrm{x}}^{*}$ defines the beam size in the horizontally focusing quadrupoles nearest to a given interaction point $[18,19]$, the value of which is critical for background optimization. The minimum value used operationally in LEP was $\beta_{\mathrm{x}}^{*}=1.25 \mathrm{~m}$.

\section{Radiation Damping and Beam Sizes}

As explained in section 3 the damping of a particle's longitudinal motion is a result of the dependence of energy loss due to synchrotron radiation on energy. Damping of transverse betatron oscillations also takes place. In the vertical plane the emission of a photon parallel to a particles trajectory does not change the betatron amplitude. However the resultant energy loss is replaced by the RF cavities and the corresponding momentum gain is, on average, in the longitudinal direction. This exchange results in a net reduction in the vertical betatron amplitude.

The situation is more complicated in the horizontal plane. In a region with dispersion when a particle emits a photon not only does its energy changes but it starts to oscillate around a different closed orbit with a corresponding change in betatron amplitude. Additionally the particle travels a greater path length during the positive part of its betatron oscillation and thus a different amount of energy is lost to radiation during the positive and negative parts of an oscillation leading to a change in amplitude. The effect of RF acceleration is as for the vertical plane. The sum of the effects is well established [8] and is encapsulated in the horizontal damping partition number described below. 


\subsection{Damping times and damping partition numbers}

Damping is thus present in all three degrees of freedom and can be characterised by damping time constants :

$$
\tau_{i}=\frac{2 E_{\mathrm{b}}}{J_{i} U_{0} f_{\mathrm{rev}}} \quad \text { with } \quad i=x, y, \varepsilon
$$

where $J_{i}$ are the damping partition numbers

$$
J_{\mathrm{x}}=1-\mathcal{D} \quad J_{\mathrm{y}}=1 \quad \text { and } \quad J_{\varepsilon}=2+\mathcal{D}
$$

where $\mathcal{D}$ is a property of the lattice configuration and is given by integration around the machine of expressions involving the the dipole field, the quadrupoles gradients and the dispersion function [20]. Note that the damping partition numbers sum to 4 .

By varying the fields experienced by the particles, typically by the introduction of wigglers magnets or shifts in the RF frequency, it is possible to change $\mathcal{D}$ and thus alter the damping rates. It can be seen that increasing the damping in the horizontal plane, say, will be at the price of reduced damping in the longitudinal plane.

\subsection{Bunch length}

The longitudinal damping described above is balanced by quantum excitation due to the statistical nature of photon emission leading to an equilibrium energy spread (see Equation (11)) or bunch length given by:

$$
\sigma_{\mathrm{l}}=\frac{\alpha_{\mathrm{c}} R}{Q_{\mathrm{s}}} \frac{\sigma_{\mathrm{e}}}{E_{\mathrm{b}}}
$$

where $Q_{\mathrm{s}}$, the synchrotron tune, gives the number of longitudinal oscillation per turn. $Q_{\mathrm{s}}$ is adjusted over the peak RF voltage $\hat{V}$ :

$$
Q_{\mathrm{s}}^{2}=\frac{\alpha_{\mathrm{c}} h}{2 \pi E_{\mathrm{b}}} \sqrt{e^{2} \hat{V}^{2}-U_{0}^{2}}
$$

Large RF voltages produce therefore a large $Q_{\mathrm{s}}$ and short bunches. The normal range of $Q_{\mathrm{s}}$ values used for LEP operation varies between 0.06 and 0.13 .

\subsection{Horizontal emittance}

Emission of discrete photons of synchrotron radiation in dispersive regions also excite random betatron oscillations with the emission of a photons changing both the amplitude and angle of the betatron motion of a particle by virtue of the change in closed orbit due to the energy shift.

Again a balance is reached between quantum excitation and damping to produce an equilibrium distribution. The corresponding horizontal emittance for an iso-magnetic storage ring is given by :

$$
\epsilon_{\mathrm{x}}=\frac{c_{\mathrm{q}} \alpha_{\mathrm{c}} R \gamma^{2}}{J_{\mathrm{x}} \rho Q_{\mathrm{x}}}
$$

where $J_{\mathrm{x}}$ is the horizontal damping partition number, $\mathrm{R}$ the radius of the machine and $Q_{\mathrm{x}}$ the horizontal tune of the machine. Given that $\alpha_{\mathrm{c}} \simeq Q_{\mathrm{x}}^{-2}$ the expression for the horizontal emittance then becomes

$$
\epsilon_{\mathrm{x}} \simeq \frac{c_{\mathrm{q}} R \gamma^{2}}{J_{\mathrm{x}} \rho Q_{\mathrm{x}}^{3}} .
$$


This relation highlights both some basic limitations faced by LEP as it moved to high energy as well as some possible ways around these limitations. It can be seen that the horizontal emittance:

- depends the geometry of the accelerator and the properties of the guide field ( $\mathrm{R}, \rho$ and $\left.J_{\mathrm{x}}\right)$,

- increases with the square of the energy,

- is inversely proportional to the third power of the horizontal tune.

One would hope to increase the energy of LEP as far as the RF system allows. However, the increase in horizontal beam size with energy poses a possible limitation to this energy increase either when the transverse beam size reaches the physical aperture imposed by the vacuum chamber or more subtlety if it approaches the dynamic aperture. Depending on the optics, the latter can be larger or smaller than the physical aperture.

It is possible to reduce the horizontal beam size by making use of the strong dependence of the emittance on the lattice functions $\left(\epsilon_{\mathrm{x}} \sim Q_{\mathrm{x}}^{-3}\right)$. Moving to a lattice with stronger focusing is very effective in reducing the horizontal beam size.

\subsection{Vertical emittance}

One would expect the vertical size beam to be negligible, it being damped almost to zero. However inevitably one has coupling, and residual vertical dispersion arising from dipole errors and the vertical separation bumps. These effects contribute to the vertical beam size and in practice, the vertical emittance of the beam is below $1 \%$ of the horizontal value.

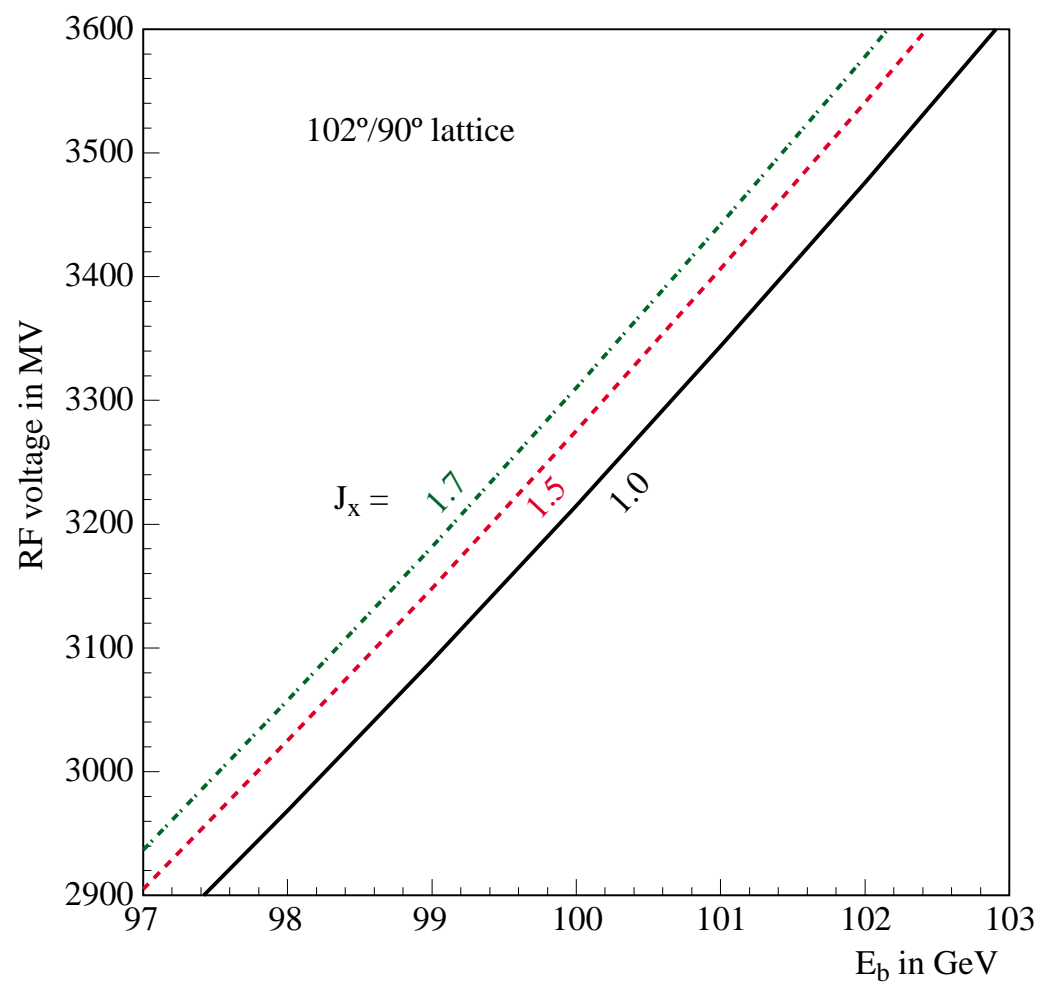

Figure 6: Maximum energy as a function of the RF voltage for different values of the horizontal damping partition number $J_{\mathrm{x}}$. The curves have been calculated for a quantum lifetime of $100 \mathrm{~h}$. 


\subsection{Variation of the damping partition numbers}

The possibility of modifying the damping partition numbers by shifting the RF frequency has been successfully used in LEP at high energies to reduce the horizontal beam size and increase the bunch length. One has

$$
\frac{\Delta p}{p}=-\frac{1}{\alpha_{\mathrm{c}}} \frac{\Delta f_{\mathrm{RF}}}{f_{\mathrm{RF}}} .
$$

It follows that the average momentum of the beam can be lowered with a positive shift of the RF frequency. In regular operation this shift is typically of the order $\Delta f_{\mathrm{RF}}=100-140 \mathrm{~Hz}$. This shift of the RF frequency induces an orbit offset in the arc quadrupoles and modifying $\mathcal{D}$ and thus the damping partition numbers. In LEP a $100 \mathrm{~Hz}$ frequency shift increases $J_{\mathrm{x}}$ to about $1.6, J_{\varepsilon}$ is reduced to around 1.4. The consequent reduction in horizontal beam size results in a luminosity increase. However, the larger energy spread necessitates an RF bucket with increased energy acceptance, see Equation (13). As a consequence, for a given voltage and quantum lifetime, the maximum achievable energy under such a configuration is slightly lowered as illustrated in Figure 6.

\section{Resonances}

The betatron tunes are important machine parameters that characterise the particle motion in the transverse planes. For an ideal machine (perfect magnets and alignment) and a perfectly monochromatic beam the values of the betatron tunes could in principle take any value that is compatible with the strength of the quadrupoles. In reality however, small errors do occur in magnetic fields and in component alignment. To avoid instabilities due to these imperfections the tune values must be chosen with great care. A simple example can be given for a dipole field error when the machine tune is an integer number. In this case a particle will always return to the location of the perturbation with the same phase relation. The kick from the dipole error will therefore systematically add from one turn to the next and the amplitude of the oscillation will grow until the particle is lost from the machine. For a quadrupolar field error, a similar accumulation of kicks happens when the tune is half an integer. When sextupoles are present in the machine, the kicks on every third turn accumulate if the tune is a multiple of one third. More generally an n-th order multi-pole field error can drive resonances up to n-th order whenever the condition

$$
q Q=m \quad \text { with } \quad|q| \leq n
$$

with $q$ and $m$ integers, is fulfilled by the machine tune $Q$. Resonances build up over a certain number of turns and their strength usually decreases with the order of the resonances.

In general the horizontal and vertical planes are never perfectly decoupled. The coupling may be produced for example by rotated quadrupoles and solenoids. For coupled motion the resonance condition becomes

$$
p Q_{\mathrm{x}}+q Q_{\mathrm{y}}=m
$$

where $p, q$ and $m$ are integers and $(|p|+|q|)$ is the order of the resonance. The lowest order coupling resonances are

$$
Q_{\mathrm{x}}+Q_{\mathrm{y}}=|m| \text { and } \quad Q_{\mathrm{x}}-Q_{\mathrm{y}}=|m| .
$$

They are respectively called the sum and difference resonances. The sum resonance is unstable, allowing the amplitudes in both planes to grow. On the difference resonance the coupled 
motion is stable and the sum of the amplitudes remains constant. But to optimize the machine performance the difference resonance must be carefully suppressed to avoid a vertical blow up of the beam due to coupling of the horizontal motion into the vertical plane. A general treatment of the resonance conditions can be found in Reference [21].

\subsection{Resonance diagram}

The undesirable tune combinations that lead to resonances can be visualised on a tune diagram as shown in Figure 7. The search for the best operational combination of tune values is complicated by the fact that the beam occupies an area in tune space called the tune footprint. When the beams are colliding the size of the footprint is mainly determined by the the beam-beam effect because the field from the counter-rotating beam acting on a particle is a strong and non-linear function of the particle amplitude. At LEP the effect of most resonances is counter-balanced by the strong damping of the oscillations that prevents the build up of resonant motion. At high energy resonances of order higher than 3 are completely suppressed. Even the resonance of third order $(Q=n+1 / 3)$ can be covered by the tune footprint without always causing problems. The performance and the particle backgrounds in the experiments are however sensitive to the position of the footprint in the tune diagram.

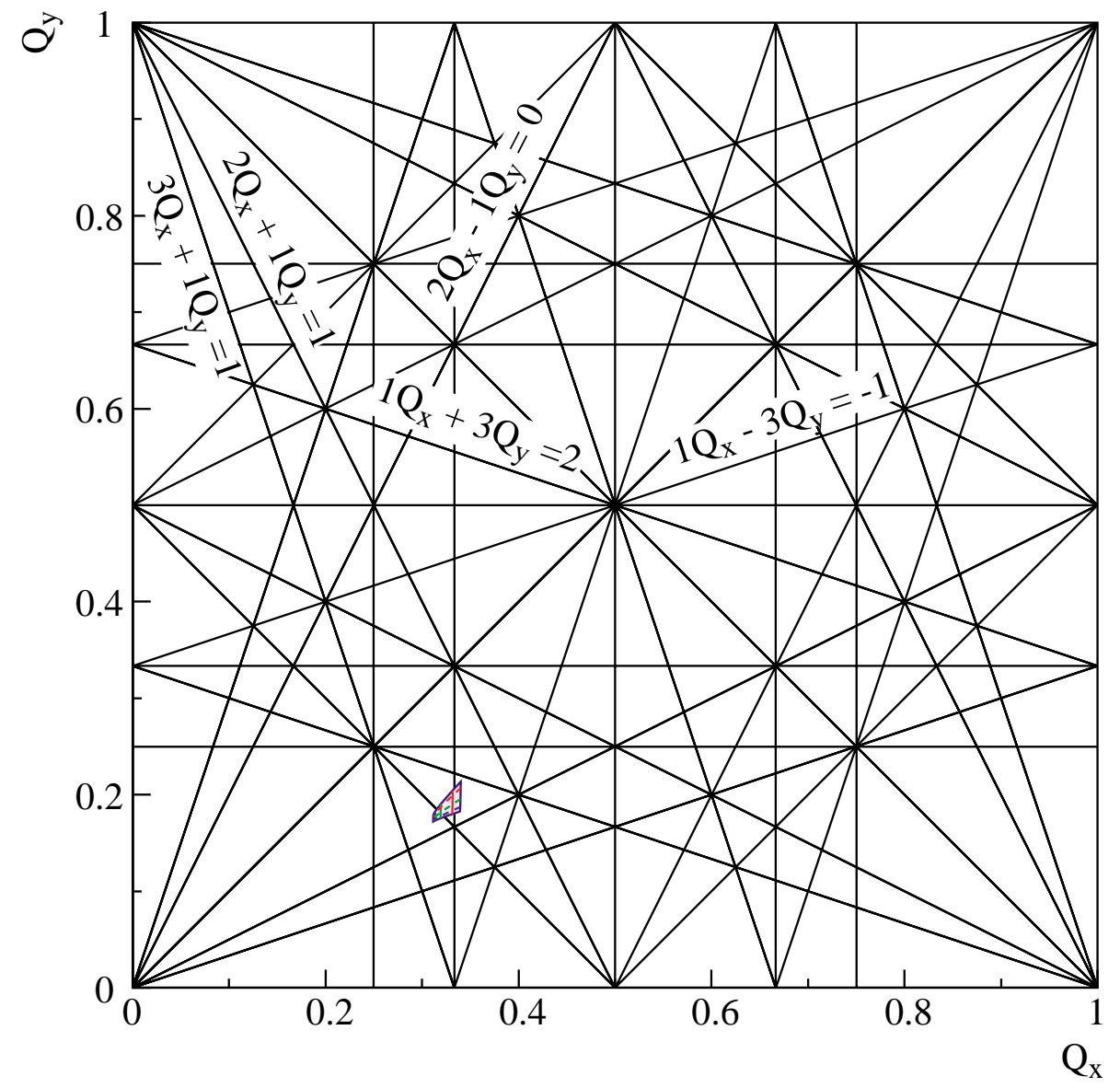

Figure 7: Illustration of a tune diagram for resonances up to 4th order. The typical tune area, occupied by a colliding beam at LEP1 is also shown as shaded area $\left(Q_{\mathrm{x}} \approx 0.31-0.34\right.$ and $\left.Q_{\mathrm{y}} \approx 0.17-0.214\right)$. 


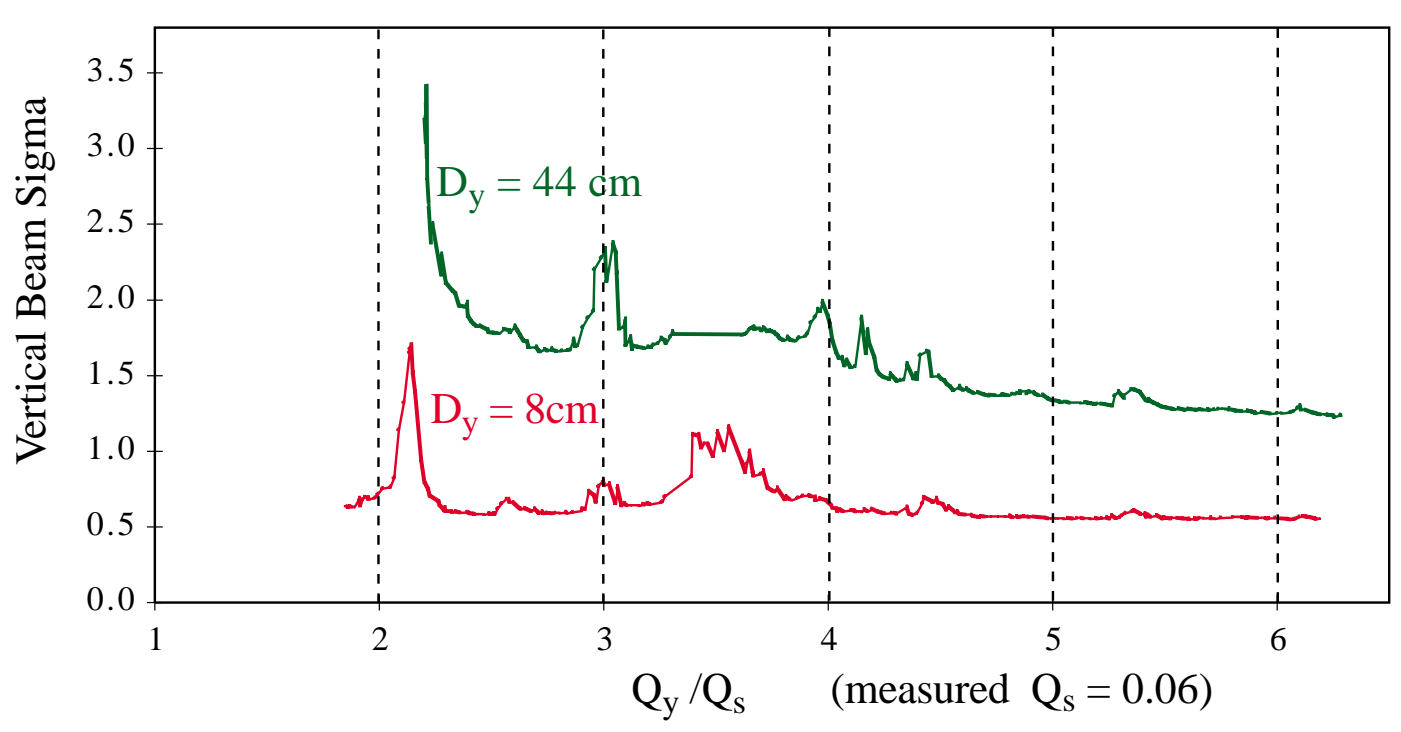

Figure 8: Vertical beam size as a function of the ratio between the fractional part of the vertical tune $Q_{\mathrm{y}}$ and the synchrotron tune $Q_{\mathrm{s}}$ for two different values of the average vertical dispersion in LEP. For a higher dispersion, the average beam size increases and the synchro-betatron resonances become significantly stronger. The position of the second synchrotron sideband $\left(Q_{\mathrm{y}} / Q_{\mathrm{s}}=2\right)$ is shifted upwards to $Q_{\mathrm{y}} / Q_{\mathrm{s}} \simeq 2.15$ due to collective effects (see section 8$)$.

\subsection{Synchro-betatron resonances}

The particle oscillations in the longitudinal plane complicate the resonance picture because they can be coupled into the transverse plane by so-called 'synchro-betatron' coupling. There are many known mechanisms for coupling the longitudinal and transverse motion of the particles. Of these, usually the most severe is dispersion at the location of RF cavities [22]. Consequently, most colliders are designed with zero dispersion at the RF cavities. However, measurements in LEP have shown that the residual dispersion, produced by machine imperfections, is significant and particularly dangerous in the vertical plane.

The mechanism for synchro-betatron coupling generated by dispersion at the RF cavities can be illustrated for a particle that arrives at an RF cavity with the same energy as the synchronous particle and no amplitude in betatron phase space. During the traversal of the cavity the particle gains energy and a new orbit is established around which the particle must oscillate. The particle which was initially at zero displacement has a new equilibrium orbit displaced from the original one by an amount equal to the product of the relative energy gain and the dispersion at the cavity. When the oscillation frequencies in the transverse planes are an integer multiple of those in the longitudinal plane, then the amplitude of the betatron oscillation builds up with each traversal of the cavity. More generally the resonant condition is

$$
k Q_{\mathrm{x}}+m Q_{\mathrm{y}}+n Q_{\mathrm{s}}=p
$$

where $k, m, n$ and $p$ are integers.

The current that may be accumulated in LEP is fundamentally limited by the transverse mode coupling instability (described in Section 8.4). In order to raise the threshold of this instability it was necessary to increase significantly the synchrotron tune $Q_{\mathrm{s}}$ at injection energy to $\approx 0.13$. Since very high values of $Q_{\mathrm{s}}$ cannot be maintained during the ramp, it is inevitable that synchro-betatron resonances must be crossed. For the normal high intensity injection the vertical tune is is maintained between the first $\left(Q_{\mathrm{y}}=p+Q_{\mathrm{s}}\right)$ and the second $\left(Q_{\mathrm{y}}=p+2 Q_{\mathrm{s}}\right)$ 
synchro-betatron resonances. Losses are avoided in the energy ramp by crossing the synchrobetatron resonances at the highest possible energy where their strength is significantly reduced. For an experimental observation of synchro-betatron resonances in LEP see Figure 8.

\section{Collective effects and impedances}

The particles contained in a high intensity beam represent a sizeable charge and current which act as a source of electro-magnetic fields. The fields are shaped by the boundary conditions imposed by the beam surroundings (vacuum chambers, cavities, etc) and can act back on the beam as sketched in Figure 9. The feedback of the fields on the beam can lead to frequency shifts (change of betatron or synchrotron tunes) and to resonant increase of small beam disturbances, i.e. instabilities. These phenomena are called collective effects since they are caused by the collective action of the particles in the bunch.

The impedance $Z(w)$ of an accelerator element is defined as the ratio between the voltage induced in the element and the circulating current and is given by

$$
Z(\omega)=\frac{V(\omega)}{I(\omega)}
$$

$Z(\omega)$ is a function of the excitation frequency $\omega$ and is in general a complex number. The real or resistive part of $Z(\omega)$ leads to energy losses while the imaginary or reactive component causes frequency shifts. The vacuum chamber of an accelerator is too complicated to obtain an analytical expression for its impedance and in general each section is treated separately. There are however a few classes of impedances which are common to all accelerators. Cavitylike objects can be described by a resonance frequency and a quality factor Q. Broad Band impedances with low $\mathrm{Q}$ value span a large frequency range, while narrow band impedances due to high $\mathrm{Q}$ value cavities only cover a limited frequency span. An example for an impedance spectrum is shown in Figure 10.

In the longitudinal plane, the voltage induced by the charged particles passing such an impedance produces additional energy losses. In the transverse planes a beam travelling offcentre through an object generates deflecting forces which act back on the transverse motion.

The influence of collective effects increases with beam intensities and may eventually limit the maximum intensities which can be accumulated. Collective effects therefore impose additional constraints to those discussed previously, such as lattices resonances. A rather complete coverage of the different aspects of collective effects is presented in Reference [23].

\subsection{The loss factors}

The convolution of the impedance with the frequency spectrum $h(\omega)$ of the bunch is called the loss factor

$$
k \sim \int Z(\omega) h(\omega) d w .
$$

In the longitudinal plane, the loss factor $k_{\|}\left(\sigma_{1}\right)$ expresses the energy lost by the bunch due to the impedances. The loss factor is a strong function of the bunch length $\sigma_{1}($ through $h(\omega))$ and is largest for short bunches. The transverse effects are similarly expressed by the transverse loss factor $k_{\perp}\left(\sigma_{1}\right)$ which represents the transverse force per unit charge integrated over one revolution. 

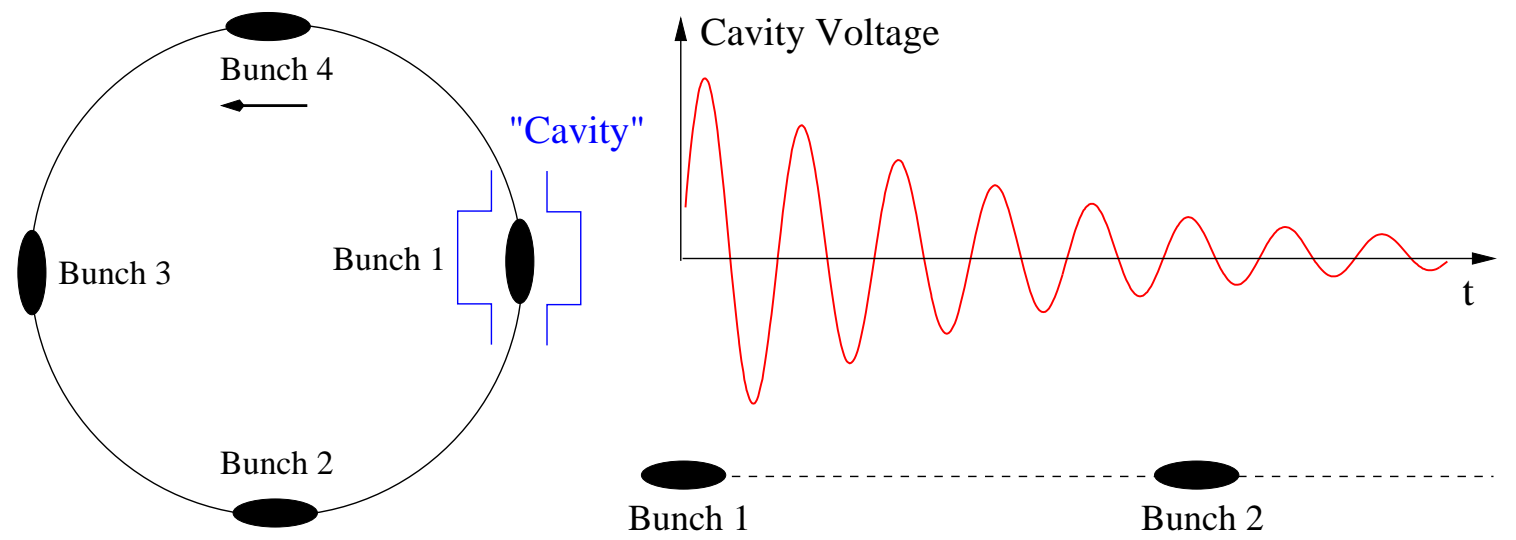

Bunch 1

Bunch 2

Figure 9: Each bunch induces a field in the cavity which oscillates and slowly decays as the energy is dissipated in the cavity walls. The bunch may be influenced by its own field and, if the decay time is very slow, the next bunch or the same bunch on the next turn may find some field left.

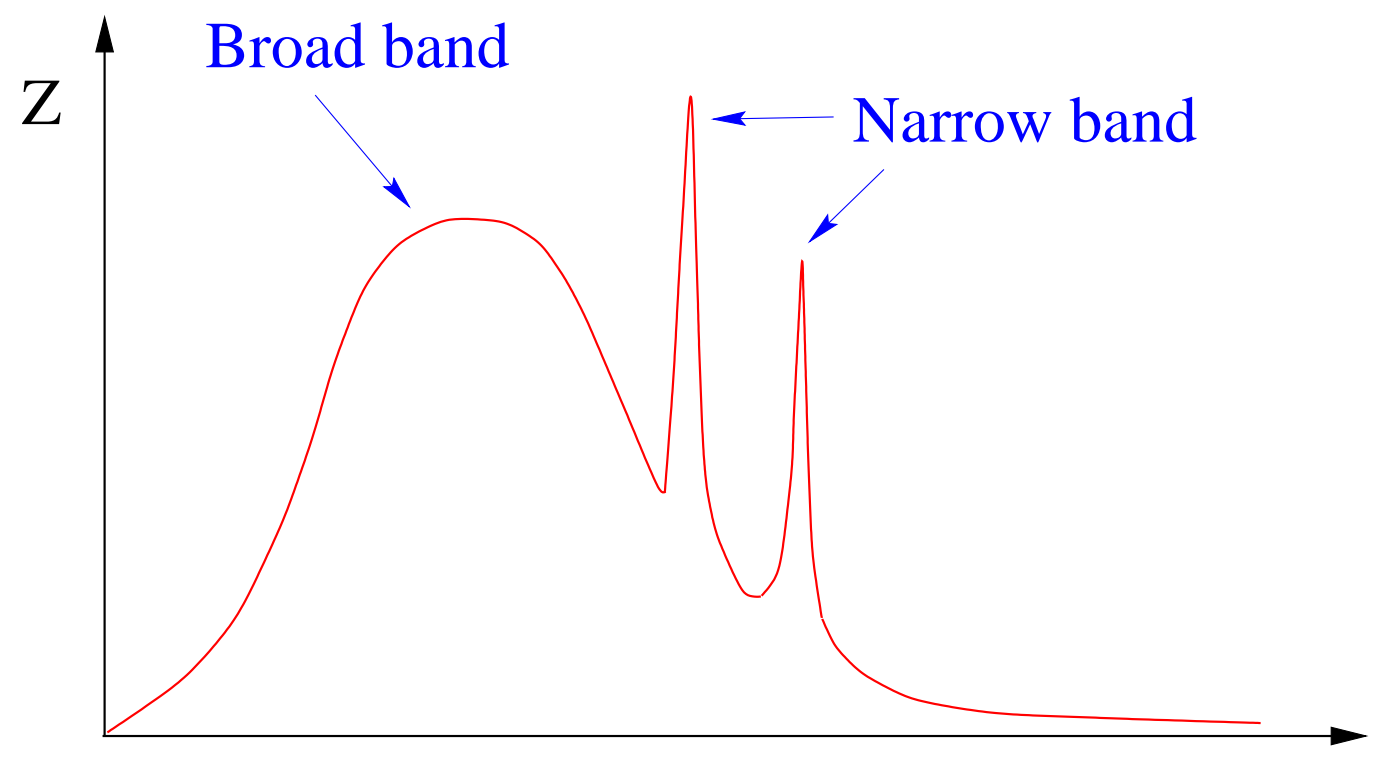

$\omega$

Figure 10: Qualitative spectra of broad band (low Q value) and narrow band (high Q value) impedances. Narrow band impedances are typically due to high quality resonators like RF cavities. 


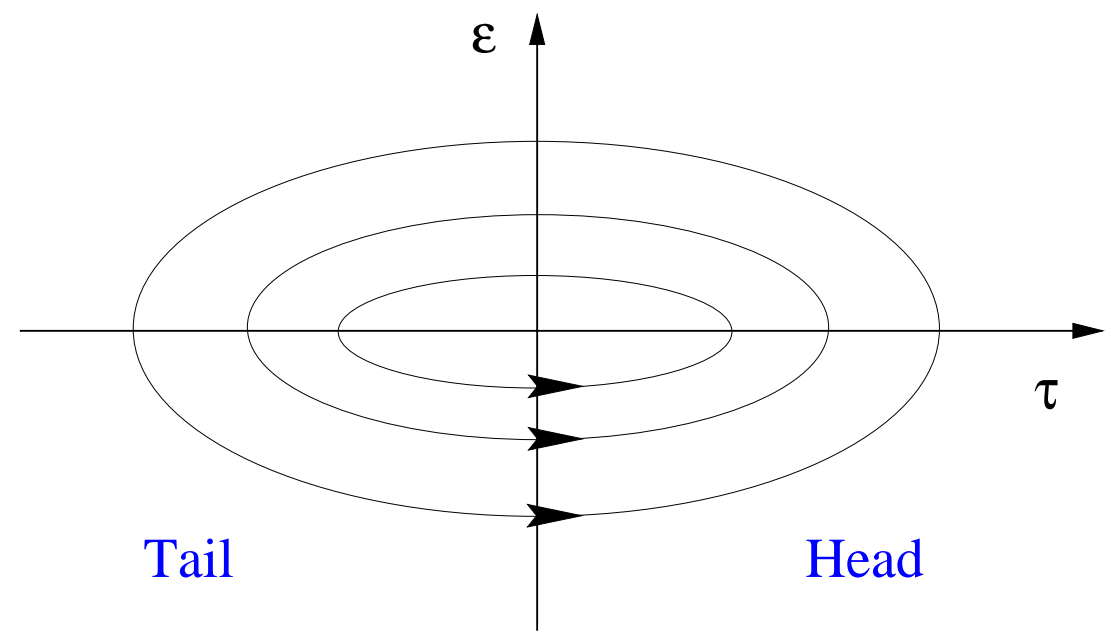

Figure 11: Phase space trajectories in momentum $(\epsilon)$ and time $(\tau)$ around the synchronous particle.

\subsection{Parasitic mode losses}

In the longitudinal plane the voltages induced by the bunch in the different parts of the machine cause the bunch to lose energy. The power loss from the two beams is

$$
P_{\mathrm{par}}=2 k_{\mathrm{b}} f_{\mathrm{rev}} I_{\mathrm{b}}^{2} k_{\|}\left(\sigma_{\mathrm{l}}\right) \text {. }
$$

This expression is valid when the fields excited by one bunch have decayed before the next bunch arrives. If this is not the case, coherent addition of fields may lead to a larger energy loss, proportional to the square of the number of interacting bunches. It is rather straightforward to evaluate the energy lost by the beam in each individual element contributing to the impedance. But it is more difficult to predict where the energy will be deposited in the machine, particularly for the high frequency component which can travel long distances with little attenuation. It is therefore extremely important to identify the critical locations (e.g. transitions of the vacuum chamber) and to take adequate counter measures (cooling, absorbers).

\subsection{Head-tail instability}

The classical "head-tail instability" involves three principal phenomena in accelerators. Firstly the synchrotron motion, where the momenta and the time delay of the particles oscillate with respect to a reference particle. The oscillations may be depicted as closed trajectories (centred on the reference particle) in momentum-time phase space (Figure 11). A particle with zero momentum deviation may therefore be situated ahead of the synchronous particle (in the head of the bunch) or behind it (in the tail). This situation is reversed after one half of a synchrotron period when the particles interchange positions at the head and tail of the bunch. The second ingredient is the chromaticity by which particles with different momenta have different betatron oscillation frequencies (tunes). As particles oscillate in energy (momentum) they therefore oscillate in betatron tune. Also, particles with zero energy deviation, which are situated at the head and tail of the bunch, have the same betatron tunes. Consequently a particle starting its synchrotron motion at the head of the bunch has the same momentum deviation as the synchronous particle and therefore the same betatron frequency. As this particle moves in synchrotron phase space, the betatron frequency initially decreases (assuming a negative value of chromaticity) and a betatron phase lag develops. When the particle reaches the tail of the 
bunch its phase lag is at a maximum. Continuing the motion towards the head of the bunch causes the particle to gradually regain betatron phase until it reaches the head of the bunch where the whole cycle restarts. It is also apparent that, if a large number of particles were distributed around the synchronous orbit then the betatron phase pattern would be stationary. The net result of all this is that the head and the tail of the bunch oscillate at the same frequency but with a phase difference. The third ingredient needed for the head-tail instability is a high frequency impedance. In the presence of such an impedance, the head of the bunch will induce transverse electro-magnetic fields which will be experienced by the particles in the tail of the bunch. The phase difference between the head and the tail accompanied by the continuous exchange of particles between head and tail may lead to a situation of positive feedback and instability in the particle motion.

It has been shown that the transverse motion of certain modes of oscillation may be stable or unstable depending on the sign of chromaticity. For LEP the "0" mode where head and tail move in phase is unstable for negative values of chromaticity. However for high positive values of chromaticity the "- 1 " mode where the head and the tail move in anti-phase is unstable. Consequently LEP has been operated with a low positive value of chromaticity usually in the range of 0 to +2 .

\subsection{Transverse mode coupling instability}

In the previous section the classical head-tail instability was shown to be a resonant effect driven by broad band impedances and controlled by the chromaticity. The "strong headtail instability" or "transverse turbulent instability" or more correctly the "transverse mode coupling instability" is a non-resonant instability which is unaffected by chromaticity and can be a severe and fundamental limitation to the intensity. This instability was first observed, but not understood in SPEAR, and later observed and explained in the PETRA machine [24, 25] and in parallel studied by other authors [26]. The mechanism is identical to the classic head-tail instability in that the synchrotron motion is needed to interchange the head and tail of the bunch to drive the instability. However in the case of TMCI the transverse forces created by the particles in the head of the bunch and experienced by those in the tail can be strong enough to modify the frequencies of the modes of oscillation ( 0 and -1 in particular). At vanishing bunch intensities these modes are separated by the synchrotron frequency, however as the intensities are increased the modes converge in frequency. The threshold of the instability is reached when the two modes have the same frequency and become degenerate as shown in Figure 12.

The threshold bunch intensity required for the onset of the instability is given approximately by

$$
I_{\mathrm{th}} \simeq \frac{8 f_{\mathrm{rev}} Q_{\mathrm{s}} E_{\mathrm{b}}}{e \sum_{i}\left(\beta_{i} k_{\perp i}\left(\sigma_{\mathrm{l}}\right)\right)}
$$

where $\beta_{i}$ the average value of the $\beta$ function at the $\mathrm{i}$-th impedance and $k_{\perp i}$ is the transverse loss factor of the i-th impedance. This expression is valid in both planes, but usually the vertical plane is the more critical, because the vertical dimensions of the vacuum chamber are usually smaller than the horizontal ones which leads to larger vertical impedances.

The possibilities to delay the onset of this instability are evident by inspection of Equation (39). During the years of operation of LEP the threshold for the TMCI has been steadily increased. The injection energy $\left(E_{\mathrm{b}}\right)$ has been increased from 20 to $22 \mathrm{GeV}$ by upgrading the $\mathrm{RF}$ system in the injector to LEP (the SPS). The value of the synchrotron tune $\left(Q_{\mathrm{s}}\right)$ has been increased from its design value of 0.08 to a maximum value of 0.13 . This increase of $Q_{\mathrm{s}}$ has two unwanted side effects : the shorter bunch length increases the loss factor $k_{\perp}$ and the tune operating point is more delicate. The decrease in bunch length was nullified by using spe- 


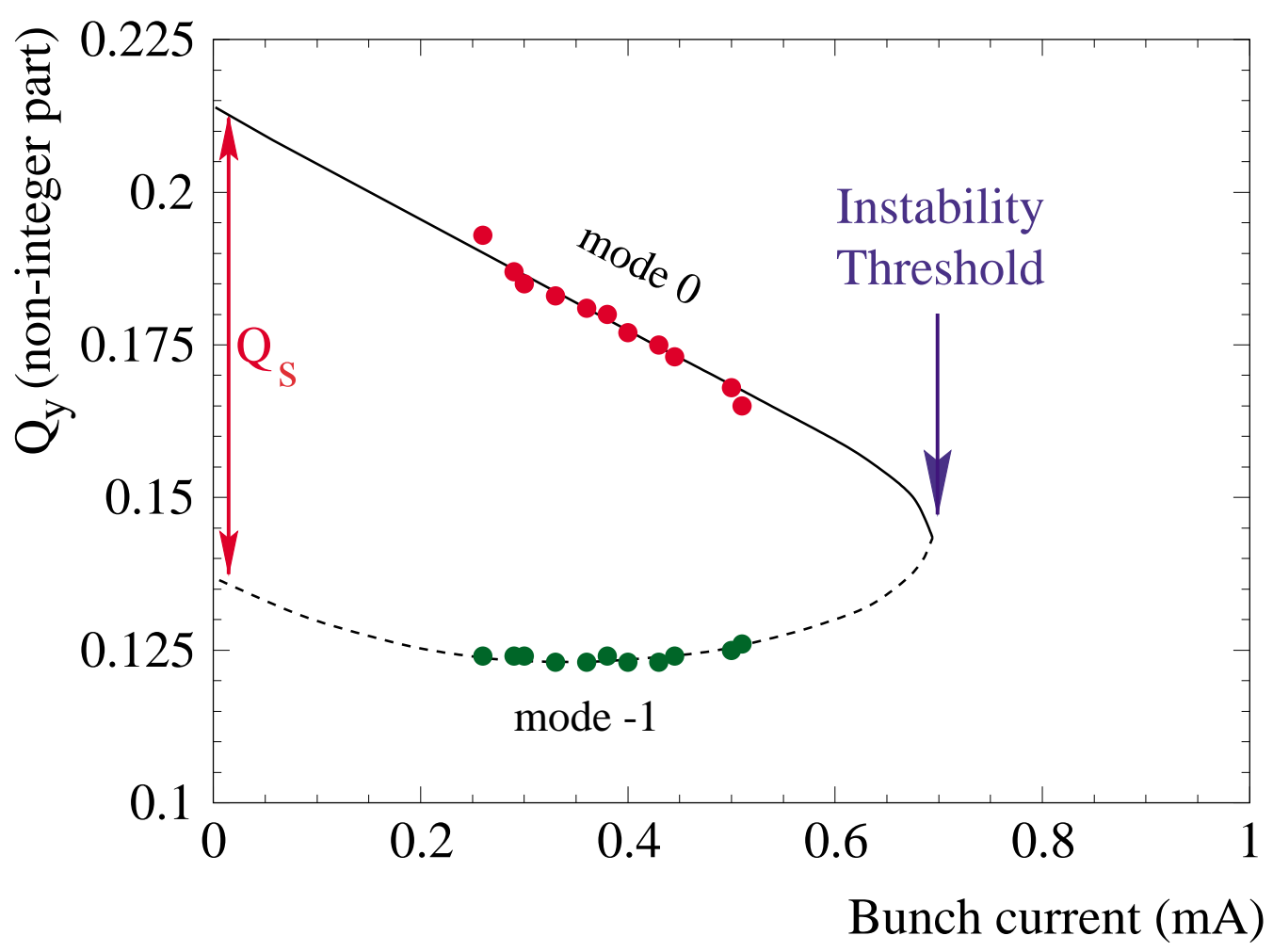

Figure 12: Measurement of the 0 and -1 modes of oscillation as a function of the bunch current at LEP for $Q_{\mathrm{s}}=0.082$. As the current increases the two modes approach until they merge at the instability threshold.

cial wiggler magnets at full strength at injection. During the design of LEP, the transverse impedance seen by the beam $\left(k_{\perp}\right)$ was strictly controlled and minimized by measuring and if necessary redesigning each element before installation in the ring. The major part of the residual impedance resulted from the necessary installation of the room temperature copper RF cavities for LEP. The design of the LEP super-conducting RF cavities allowed for a very low transverse impedance. Hence super-conducting cavities were gradually installed in the location of the original copper cavities thereby greatly reducing the transverse impedance. In addition the betatron functions $\left(\beta_{i}\right)$ were systematically examined and a great effort was made to reduce the local betatron function at elements with significant transverse impedance.

As a net result of these efforts the threshold for TMCI has been raised by around a factor of two. Nevertheless it still remains a fundamental limitation to the LEP bunch current at injection energy. The present limitation is around $1.0 \mathrm{~mA} /$ bunch.

\subsection{Coupled bunch instability}

Coupled bunch instability becomes relevant when the fields created by a bunch can perturb the motion of the following bunches. To generate an effect on more than one bunch, the fields must persist at least until the next bunch arrives at the location of the impedance. Multi bunch instabilities are therefore mainly expected in machines containing high $\mathrm{Q}$ value, narrow band impedances like accelerating cavities. In LEP, operation with 4 or 8 equidistant bunches was not affected by this type of instability, because of the large bunch spacing. For machines with a large number of bunches, a feedback system is required to provide sufficient stability. 


\section{Beam-beam interaction and limits}

The importance of the beam-beam interaction as a performance limitation to colliding beam electron-positron storage rings was well established before the construction of LEP. A review with observations from many machines was compiled in 1985 [27]. A purely analytical treatment of the beam-beam interaction is difficult due to the complexity of the forces and the high number of particles involved. Simulation techniques have gained in importance with the development of detailed codes and the availability of more powerful computers. For a review at the time of LEP planning and construction see [28]. These studies strongly influenced the design of LEP : the symmetry between the four interaction regions, the choice of the optics, the tune working point and the installation of wiggler magnets.

\subsection{Beam-beam parameters}

Associated with each bunch is a strong non-linear electro-magnetic field which deflects individual particles of the counter-rotating beam at each collision, see Figure 13. For small displacements from the centre of the bunch this field varies linearly with distance, like a normal quadrupolar lens, whereas at larger displacements the field is highly non-linear. The nonlinearity of the beam-beam force is the main source of performance limitations. The non-linear fields cause beam size blow up and drive higher order resonances.
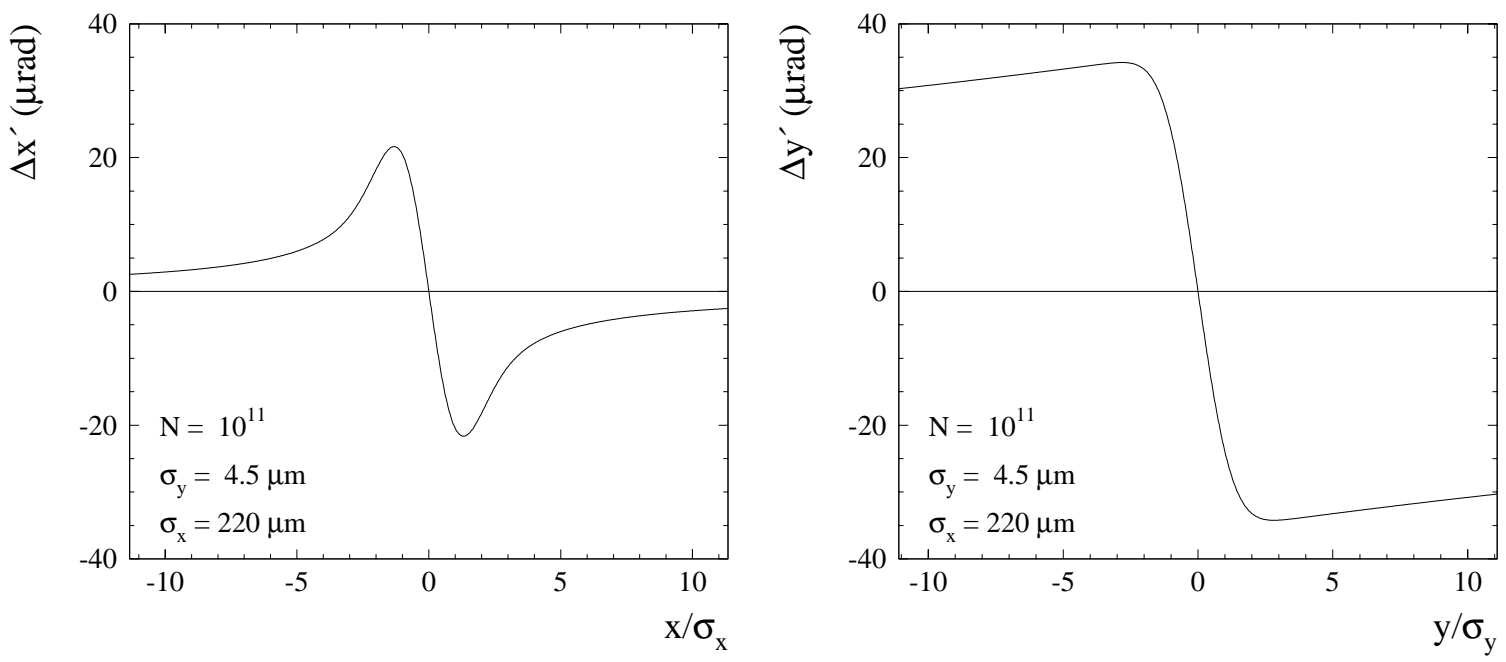

Figure 13: Beam-beam deflection angles of a single particle as a function of the horizontal (left) and the vertical distance (right) to the centre of a counter-rotating bunch with a population of $10^{11}$ particles at a beam energy of $45.6 \mathrm{GeV}$. The distance is given in units of r.m.s. beam sizes. Close to the centre, the deflection angles grow linearly with the distance.

The beam-beam force produces a tune spread which is described by the beam-beam strength parameter, also referred to as beam-beam tune shift. This parameter quantifies the effect of the linear beam-beam field at the centre of the bunch, but is also representative of the nonlinear effects, since the magnitudes of the linear and non-linear components are proportional to each other. The tune shift is largest for particles in the centre of the bunch and vanishes for particles at large amplitudes. The general expression for the beam-beam parameters, with different beam sizes and intensities for the two beams, can be written as :

$$
\xi_{\mathrm{x}+}=\frac{r_{\mathrm{e}} N_{\mathrm{b}-} \beta_{\mathrm{x}+}^{*}}{2 \pi \gamma_{+} \sigma_{\mathrm{x}-}\left(\sigma_{\mathrm{x}-}+\sigma_{\mathrm{y}-}\right)}
$$




$$
\xi_{\mathrm{y}+}=\frac{r_{\mathrm{e}} N_{\mathrm{b}-} \beta_{\mathrm{y}+}^{*}}{2 \pi \gamma_{+} \sigma_{\mathrm{y}-}\left(\sigma_{\mathrm{x}-}+\sigma_{\mathrm{y}-}\right)}
$$

where the labels $(+)$ and $(-)$ refer to the $\mathrm{e}^{+}$and $\mathrm{e}^{-}$beams. The $\mathrm{e}^{-}$beam parameters are obtained with the substitution $+\leftrightarrow-$. These formulae apply to head-on collisions of Gaussian shaped bunches. Since LEP is a symmetric machine $\left(\beta_{-}^{*}=\beta_{+}^{*}\right.$ in both planes) filled with equal $\mathrm{e}^{+}$and $\mathrm{e}^{-}$currents, the beam-beam tune shifts of the two beams are equal, i.e. $\xi_{\mathrm{x}}=\xi_{\mathrm{x}+}=\xi_{\mathrm{x}-}$ and $\xi_{\mathrm{y}}=\xi_{\mathrm{y}+}=\xi_{\mathrm{y}-}$. For flat beams $\left(\sigma_{\mathrm{y}} \ll \sigma_{\mathrm{x}}\right)$ the beam-beam tune shifts are in a good approximation given by :

$$
\begin{gathered}
\xi_{\mathrm{x}}=\frac{r_{\mathrm{e}}}{2 \pi \gamma} \frac{N_{\mathrm{b}} \beta_{\mathrm{x}}^{*}}{\sigma_{\mathrm{x}}^{2}}=\frac{r_{\mathrm{e}}}{2 \pi \gamma} \frac{N_{\mathrm{b}}}{\epsilon_{\mathrm{x}}} \\
\xi_{\mathrm{y}}=\frac{r_{\mathrm{e}}}{2 \pi \gamma} \frac{N_{\mathrm{b}} \beta_{\mathrm{y}}^{*}}{\sigma_{\mathrm{x}} \sigma_{\mathrm{y}}} .
\end{gathered}
$$

The ratio of the horizontal and vertical beam-beam tune shifts is :

$$
\frac{\xi_{\mathrm{y}}}{\xi_{\mathrm{x}}}=\frac{\sigma_{\mathrm{x}}}{\beta_{\mathrm{x}}^{*}} \frac{\beta_{\mathrm{y}}^{*}}{\sigma_{\mathrm{y}}}=\sqrt{\frac{\beta_{\mathrm{y}}^{*} \epsilon_{\mathrm{x}}}{\beta_{\mathrm{x}}^{*} \epsilon_{\mathrm{y}}}} .
$$

The ratios $\sigma_{\mathrm{x}} / \beta_{\mathrm{x}}^{*}$ and $\sigma_{\mathrm{y}} / \beta_{\mathrm{y}}^{*}$ are the r.m.s horizontal and vertical beam divergences. Equations (41) and (43) are obtained under the assumption that the beam sizes at the interaction points are given by $\sigma=\sqrt{\beta \epsilon}$, i.e. that the contribution to the beam sizes from the product of energy spread and dispersion remains small (see Equation 16). In LEP this assumption is correct for the horizontal plane and is a good approximation for the vertical plane (see also Section 13.3.2).

It is usual in accelerator design to make the betatron function ratio $\left(\beta_{\mathrm{y}}^{*} / \beta_{\mathrm{x}}^{*}\right)$ equal to the anticipated emittance ratio $\kappa=\epsilon_{\mathrm{y}} / \epsilon_{\mathrm{x}}$, in which case, the horizontal and vertical beam-beam tune shifts are also equal. In LEP this is only approximately the case, with ratios $\xi_{\mathrm{y}} / \xi_{\mathrm{x}}$ of 1.3 or more. For equal beam-beam parameters, $\xi_{\mathrm{y}}=\xi_{\mathrm{x}}$, Equation (42) may be written

$$
\xi_{\mathrm{y}}=\frac{N_{\mathrm{b}} r_{\mathrm{e}}}{2 \pi \gamma \epsilon_{\mathrm{x}}}
$$

and the horizontal emittance $\epsilon_{\mathrm{x}}$ can be approximated by

$$
\epsilon_{\mathrm{x}}=\frac{\sigma_{\mathrm{x}}^{2}}{\beta_{\mathrm{x}}} \simeq \frac{c_{\mathrm{q}} R}{\rho} \frac{\gamma^{2}}{Q_{\mathrm{x}}^{3}} .
$$

Combining Equations (44) and (45) yields

$$
\xi_{\mathrm{y}}=\frac{N_{\mathrm{b}} r_{\mathrm{e}} \rho Q_{\mathrm{x}}^{3}}{2 \pi c_{\mathrm{q}} R \gamma^{3}} .
$$

For a given energy, Equation (46) shows that $\xi$ can be a priori increased with a stronger horizontal focusing. For LEP the horizontal phase advance (and therefore $Q_{\mathrm{x}}$ ) has been progressively increased from $60^{\circ}$ to $90^{\circ}$ and finally $102^{\circ}$ per cell. The later optics was specially designed for operation at the highest beam energies, to counter-balance the energy dependence of $\xi$.

Since the beam-beam parameter decreases with the third power of the beam energy, beambeam effects become excessively strong at injection energy. For LEP the beam-beam tune spread would reach $\sim 1.25$ if the beams were to collide with the design currents during injection. To avoid problems with beam-beam effects during accumulation, the beams are separated at the collision points by electrostatic plates. Experiments have demonstrated that the current per bunch is limited to $\sim 0.03 \mathrm{~mA}$ (compared to the design figure of 0.75 ) when the beams are not separated during injection. 


\subsection{Luminosity and beam-beam limits}

The luminosity per interaction region can be expressed in terms of $\xi_{\mathrm{y}}$ (see also Equations 19 and 20):

$$
\mathcal{L}=\frac{N_{\mathrm{b}}^{2} f_{\mathrm{rev}} k_{\mathrm{b}}}{4 \pi \sigma_{\mathrm{x}} \sigma_{\mathrm{y}}}=\frac{\gamma N_{\mathrm{b}} f_{\mathrm{rev}} k_{\mathrm{b}} \xi_{\mathrm{y}}}{2 r_{\mathrm{e}} \beta_{\mathrm{y}}^{*}} .
$$

Experimentally the beam-beam interaction is characterised by three regimes.

- For low bunch currents, the beam sizes are not affected by the beam-beam forces : $\xi_{\mathrm{x}}$ and $\xi_{\mathrm{y}}$ increase linearly with current and the luminosity with the current squared.

- Above a certain threshold current, the beam-beam interaction blows up the beam sizes and the beam-beam tune shifts saturate at some value (about 0.04 at LEP1 energies). This transition is referred to as the soft (or first) beam-beam limit, above which emittances and luminosity increase linearly with current. The highest luminosity performance is obtained with continuous operation at the beam-beam limit (at maximum $\xi$ ).

- The second (or hard) beam-beam limit is reached when the beam sizes have increased to the maximum size allowed by the aperture. Above this value, the beam lifetime decreases dramatically.

Operation of a machine above the first beam-beam limit may lead to asymmetric blow-up ("flipflop") of the beams with a loss in luminosity and larger backgrounds. The flip-flop state can be understood qualitatively : it is triggered by an asymmetry between the beams, for example a difference in bunch population or in beam emittance (due to different closed orbits). In such a situation the beam-beam tune shifts of the beams differ and the beam with higher $\xi$ values blows up more. Assuming that $\mathrm{e}^{-}$beam has a larger size, its smaller current and field density will decrease the $\mathrm{e}^{+}$beam-beam parameters even further. The $\mathrm{e}^{+}$beam size may shrink more and have an even stronger effect on the $\mathrm{e}^{-}$beam, enhancing the $\xi$ values split. To avoid such a flip-flop state and obtain the best performances, it is for example important that the bunch populations of the two beams are not too different.

LEP has been operated with colliding beams at beam energies around $45 \mathrm{GeV}$ (LEP1), $65 \mathrm{GeV}$ and 80 to $101 \mathrm{GeV}$ (LEP2), thus covering a factor of two in beam energies [29, 30]. As originally anticipated, the beam-beam interaction has been a much more stringent limit at the lower and intermediate energies. Figure 14 illustrates the typical behaviour of the beam-beam tune shifts as function of the bunch current in LEP at different energies. The horizontal tune shift $\xi_{\mathrm{x}}$ was generally significantly lower than $\xi_{\mathrm{y}}$. Table 3 lists beam parameters recorded in LEP at the times of maximum vertical beam-beam parameters.

\subsection{Beam-beam effects at LEP1}

From 1989 to 1995 LEP was operated at beam energies close to $45.6 \mathrm{GeV}$ for the production of $\mathrm{Z}$ bosons [7]. The vertical beam-beam tune shift was gradually lifted above 0.04 after several years. Best performances were obtained with a $90^{\circ} / 60^{\circ}$ optics which was introduced in 1993. For this horizontal phase advance the natural horizontal emittance is $12 \mathrm{~nm}$ and the first beam-beam limit is reached at bunch currents of $\simeq 0.1 \mathrm{~mA}$, see Figure 14 .

The side effects from a too strong beam-beam interaction, such as flip-flop or excessive nonGaussian tails with background and lifetime problems, were avoided by controlled emittance increase. At LEP wiggler magnets placed in a dispersive region, so called "emittance wigglers", are able to blow up $\epsilon_{\mathrm{x}}$ from 12 to $36 \mathrm{~nm}$ at $45 \mathrm{GeV}$. In practice the beams are brought into collision with the maximum possible emittance. As the current decreases during a fill, the emittance wiggler fields are gradually turned down. The best compromise found during LEP 


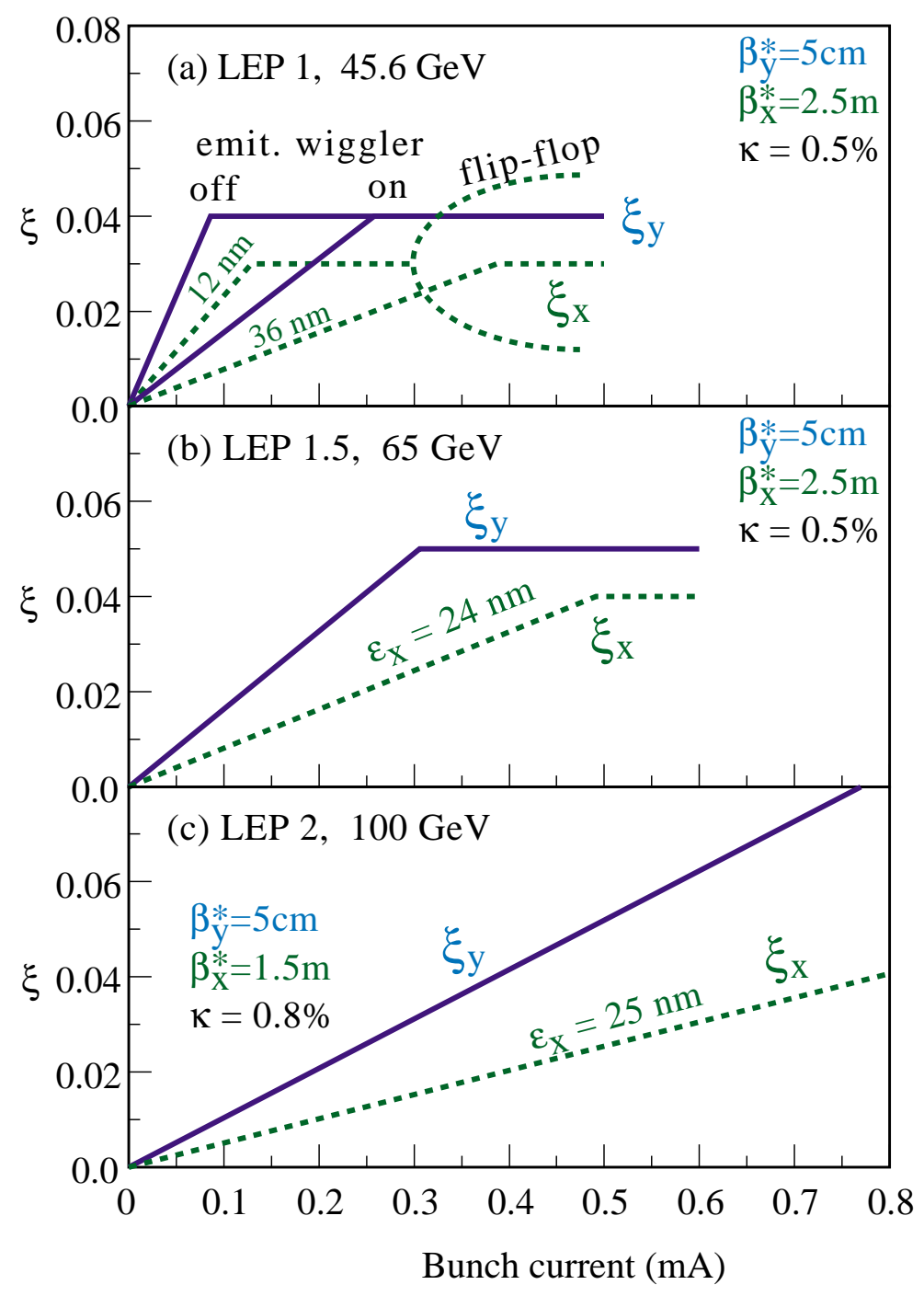

Figure 14: Schematic evolution of the beam-beam tune shifts $\xi_{\mathrm{x}}$ (dashed line) and $\xi_{\mathrm{y}}$ (solid line) with current, as typically observed in LEP at different energies. Operation with too small horizontal emittances at LEP1 (a) can drive the beams into a flip-flop state, where one beam is strongly blown up while the other beam becomes very small. At LEP2 (c), the tune shift shows practically no sign of saturation. 
Table 3: LEP beam parameters corresponding to the best performances at three different energies. The luminosities and beam-beam tune shifts are averaged over a time interval of 15 minutes. For each beam energy, the first line corresponds to the horizontal, the second line to the vertical plane.

\begin{tabular}{cccccccccc}
\hline $\begin{array}{c}E_{\mathrm{b}} \\
(\mathrm{GeV})\end{array}$ & $\begin{array}{c}N_{\mathrm{b}} \\
\left(\times 10^{11}\right)\end{array}$ & $k_{\mathrm{b}}$ & $\begin{array}{c}\mathcal{L} \\
\left(\mathrm{cm}^{-1} \mathrm{~s}^{-2}\right)\end{array}$ & $Q_{\mathrm{s}}$ & $Q$ & $\begin{array}{c}\beta^{*} \\
(\mathrm{~m})\end{array}$ & $\begin{array}{c}\epsilon \\
(\mathrm{nm})\end{array}$ & $\begin{array}{c}\sigma \\
(\mu \mathrm{m})\end{array}$ & $\xi$ \\
\hline 45.6 & 1.18 & 8 & $1.51 \times 10^{31}$ & 0.065 & 90.31 & 2.0 & 19.3 & 197 & 0.030 \\
& & & & & 76.17 & 0.05 & 0.23 & 3.4 & 0.044 \\
65 & 2.20 & 4 & $2.11 \times 10^{31}$ & 0.076 & 90.26 & 2.5 & 24.3 & 247 & 0.029 \\
& & & & & 76.17 & 0.05 & 0.16 & 2.8 & 0.051 \\
97.8 & 4.01 & 4 & $9.73 \times 10^{31}$ & 0.116 & 98.34 & 1.5 & 21.1 & 178 & 0.043 \\
& & & & & 96.18 & 0.05 & 0.22 & 3.3 & 0.079 \\
\hline
\end{tabular}

operation between luminosity and background was obtained by keeping $\xi_{\mathrm{x}}$ below 0.04 . An increase in the horizontal beam size also reduces the strength of the beam-beam effect in the vertical plane, see Equations (40) and (42) and there was no need to artificially increase the vertical emittance. Increasing the horizontal emittance does not necessarily decrease $\xi_{\mathrm{y}}$ and the luminosity. When LEP is operated at the beam-beam limit, $\xi_{\mathrm{y}}$ remains essentially constant and the vertical beam size is determined by the beam-beam interaction. Since $\xi_{\mathrm{y}} \propto 1 /\left(\sigma_{\mathrm{x}} \sigma_{\mathrm{y}}\right)$, an increase of $\sigma_{\mathrm{x}}$ is compensated by a reduction of $\sigma_{\mathrm{y}}$. A new equilibrium is reached with a smaller vertical beam size and nearly the same $\xi_{\mathrm{y}}$ and the luminosity.

When $\beta_{\mathrm{x}}^{*}$ was increased from $1.25 \mathrm{~m}$ to $2.5 \mathrm{~m}$ to reduce backgrounds in the experiments, neither the luminosity nor $\xi_{\mathrm{y}}$ decreased. This confirms the dependence of the luminosity on $\xi_{\mathrm{y}}$ alone, derived for flat beams in Equation (47). The situation is similar, but not identical to the controlled increase of the horizontal emittance. In both cases, by working at the beam-beam limit, $\xi_{\mathrm{y}}$ remains constant and the larger horizontal beam size is compensated by a decrease in vertical beam size. In contrary to the horizontal emittance increase, a larger $\beta_{\mathrm{x}}^{*}$ does not reduce $\xi_{\mathrm{x}}$ and is therefore not expected to avoid flip-flop or tails generated for high $\xi$.

In 1993 and 1994 the number of bunches was increased from 4 to 8 bunches per beam with horizontal separations in the arcs using the Pretzel scheme (see Section 4.1.1 and Figure 3). Operation and tune control became more critical and lifetime and background problems occurred at somewhat lower bunch currents. After careful adjustments and minimization of the residual horizontal separation (in addition to regular minimization of the vertical separation), vertical beam-beam tune shifts over 0.04 were reached.

Beam-beam tune shifts obtained in Pretzel operation over a few months in 1994 are shown in Figure 15. In Figures (15) to (17), $\xi_{\mathrm{y}}$ is calculated from the measured luminosity and beam currents for the nominal $\beta^{*}$, according to Equation (47). The large spread in $\xi_{\mathrm{y}}$ at a given current visible in Figure 15 reflects the difficulty in reproducing optimal conditions. Good control of many parameters and continuous tuning is necessary to maintain the highest performance, see Section 13. Unlike previous $\mathrm{e}^{+} \mathrm{e}^{-}$colliders, differences in intensities between the various electron and positron bunches up to a level of about $10 \%$ did not cause any problems.

The performance in terms of vertical beam-beam strength decreased in 1995 when 12 bunches per beam were collided using bunch trains with vertical separation (see Section 4.1.2). The maximum value of $\xi_{\mathrm{y}}$ saturated around 0.03 . This was attributed to residual vertical separation, caused by parasitic long-range collisions, that could not simultaneously be minimized for all three bunches in a train. Despite the lower maximum value of $\xi_{\mathrm{y}}$, the luminosity reached 


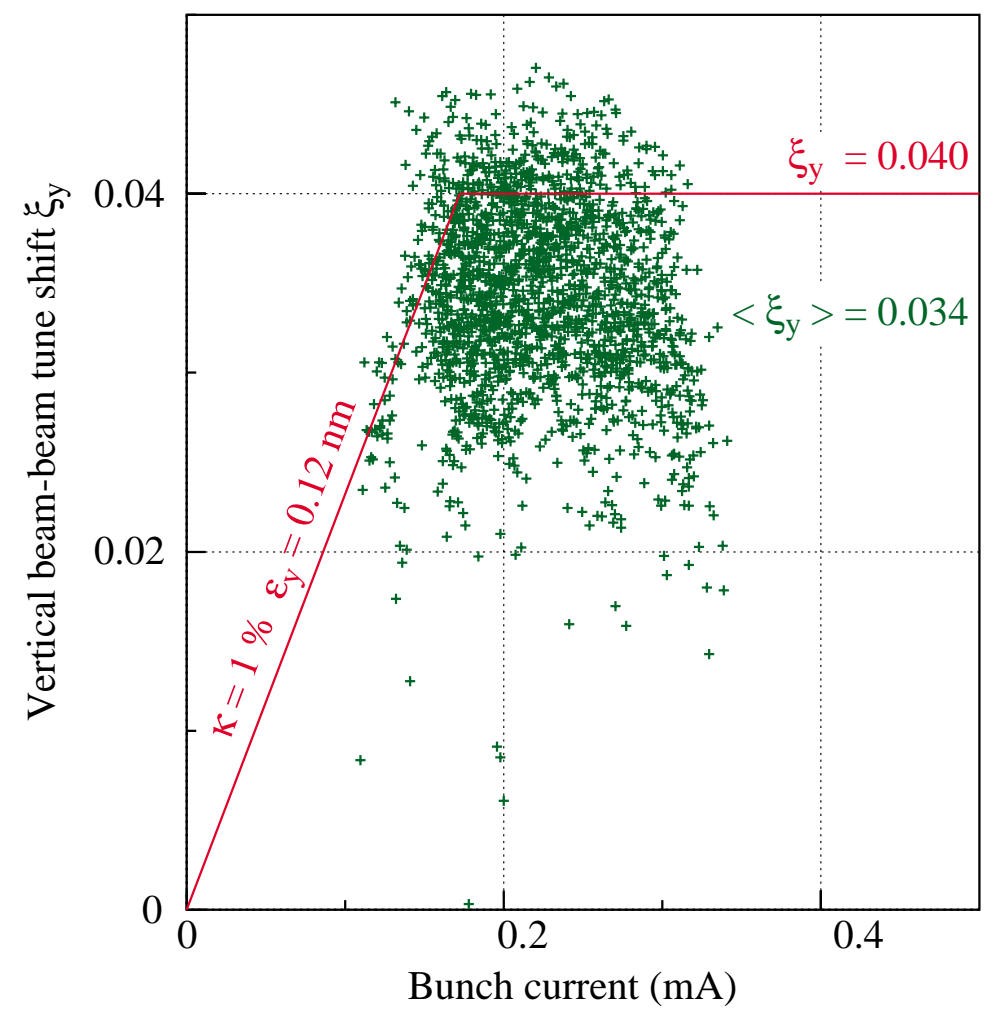

Figure 15: $\xi_{\mathrm{y}}$ dependence on bunch current in 1994 with the horizontal Pretzel scheme. The expected behaviour for an emittance ratio of $\kappa=1 \%$ and a maximum $\xi_{\mathrm{y}}=0.04$ is also shown. The average value of $\xi_{\mathrm{y}}$ is 0.034 .

new records thanks to the larger number of colliding bunches.

\subsection{Beam-beam effects at LEP1.5}

Following the installation of the first super-conducting RF cavities, the beam energy in LEP was raised to $65 \mathrm{GeV}$ in November 1995 . The $90^{\circ} / 60^{\circ}$ optics remained unchanged as compared to LEP1, but the horizontal emittance increased from $12 \mathrm{~nm}$ at $45 \mathrm{GeV}$ to $\approx 25 \mathrm{~nm}$ at $65 \mathrm{GeV}$. New record levels in beam-beam tune shifts $\left(\xi_{\mathrm{y}}=0.05\right)$ and peak luminosity $(\mathcal{L}=2.6 \times$ $10^{31} \mathrm{~cm}^{-2} \mathrm{~s}^{-1}$ ) were obtained with 4 bunches per beam during the 10 days of operation at this energy. Figure 16 shows the vertical beam-beam tune shift for a single fill at $65 \mathrm{GeV}$. The evolution of $\xi_{\mathrm{y}}$ corresponds to an emittance ratio of $\kappa=0.5 \%$. In the horizontal plane, the emittance, as observed by the synchrotron light monitor, increased only by about $10 \%$ when beams were brought into collision. The horizontal beam-beam tune shift reached $\xi_{\mathrm{x}}=0.04$.

\subsection{Beam-beam effects at LEP2}

Between 1996 and 1999 the beam energy of LEP was progressively increased from 80.5 to $101 \mathrm{GeV}$ by installing more super-conducting RF cavities and increasing the accelerating gradients.

In the first two years, LEP was operated with a $90^{\circ} / 60^{\circ}$ optics. At $86 \mathrm{GeV}$ the natural horizontal emittance reaches $42 \mathrm{~nm}$ for this optics, a value close to the maximum that can be accommodated with acceptable backgrounds in the LEP experiments. Consequently, the horizontal emittance was decreased in 1997 to about $30 \mathrm{~nm}$ at beam energies around $91.5 \mathrm{GeV}$ 


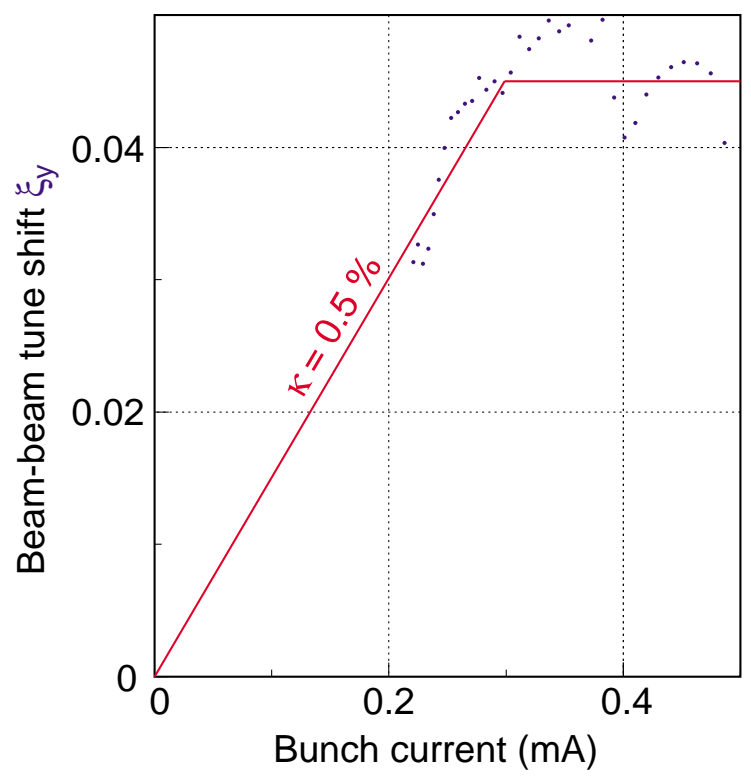

Figure 16: Evolution of $\xi_{\mathrm{y}}$ versus bunch current at a beam energy of $65 \mathrm{GeV}$ in a selected fill. The data (dots) are compared to the expected evolution for an emittance ratio $\kappa$ of $0.5 \%$ and a maximum $\xi_{\mathrm{y}}$ of 0.045 (solid line).

using a damping partition number $J_{\mathrm{x}}=1.6$. Vertical beam-beam strength tune shifts exceeded 0.05 without signs of saturation. Emittance ratios as small as $0.25 \%$ were observed for a horizontal emittance of $42 \mathrm{~nm}$.

From 1998 onwards, a $102^{\circ} / 90^{\circ}$ optics with a higher horizontal phase advance was used to reduce the horizontal emittance by about $30 \%$ compared to the $90^{\circ} / 60^{\circ}$ optics. To prevent the formation of large beam tails, the sextupole configuration of the $102^{\circ} / 90^{\circ}$ optics was adapted to obtain a small detuning with horizontal amplitude without spoiling the correction of the nonlinear chromaticity [31]. The luminosity was further boosted with horizontal damping partition numbers as high as $J_{\mathrm{x}}=1.8$. The horizontal phase advance of $102^{\circ}$ and the large horizontal damping partition number limited $\epsilon_{\mathrm{x}}$ to $25 \mathrm{~nm}$ up to a beam energy of $100 \mathrm{GeV}$.

At LEP2 the beam oscillations are strongly damped and the single particle motion has an important random walk component due to the large number of emitted photons. Consequently particles no longer lock on higher order resonances driven by the non-linear beam-beam force and the beam size blow up is reduced. Record beam-beam tune shifts of about 0.08 and luminosities of $10^{32} \mathrm{~cm}^{-2} \mathrm{~s}^{-1}$ were achieved. The observed dependence of $\xi_{\mathrm{y}}$ on current is almost linear and is shown in Figure 17. As previously, $\xi_{\mathrm{y}}$ is calculated from the measured luminosity and the unperturbed $\beta^{*}$.

At LEP the beam-beam tune shifts are extracted from the measured luminosity using Equation (47) with the nominal value of $\beta^{*}$. This is a good approximation as along as the beam-beam effects are weak, but for large $\xi$ values, the actual tune shift $\delta Q$ experienced by particles at the centre of the bunch in each collision is less than $\xi$. This is due to a reduction of the nominal $\beta^{*}$ to a smaller 'dynamic' $\beta^{*}$ by the focusing beam-beam forces. For small amplitudes the beambeam effect corresponds to an additional quadrupole and the correction can be calculated from linear optics. For $n_{\mathrm{c}}$ symmetrically spaced interaction points, $\delta Q$ and $\xi$ are related by [32] :

$$
\cos \left(2 \pi \frac{Q+\delta Q}{n_{\mathrm{c}}}\right)=\cos \left(2 \pi \frac{Q}{n_{\mathrm{c}}}\right)-2 \pi \xi \sin \left(2 \pi \frac{Q}{n_{\mathrm{c}}}\right)
$$

with $Q$ the nominal machine tune (without beam-beam). In the vertical plane LEP operates 


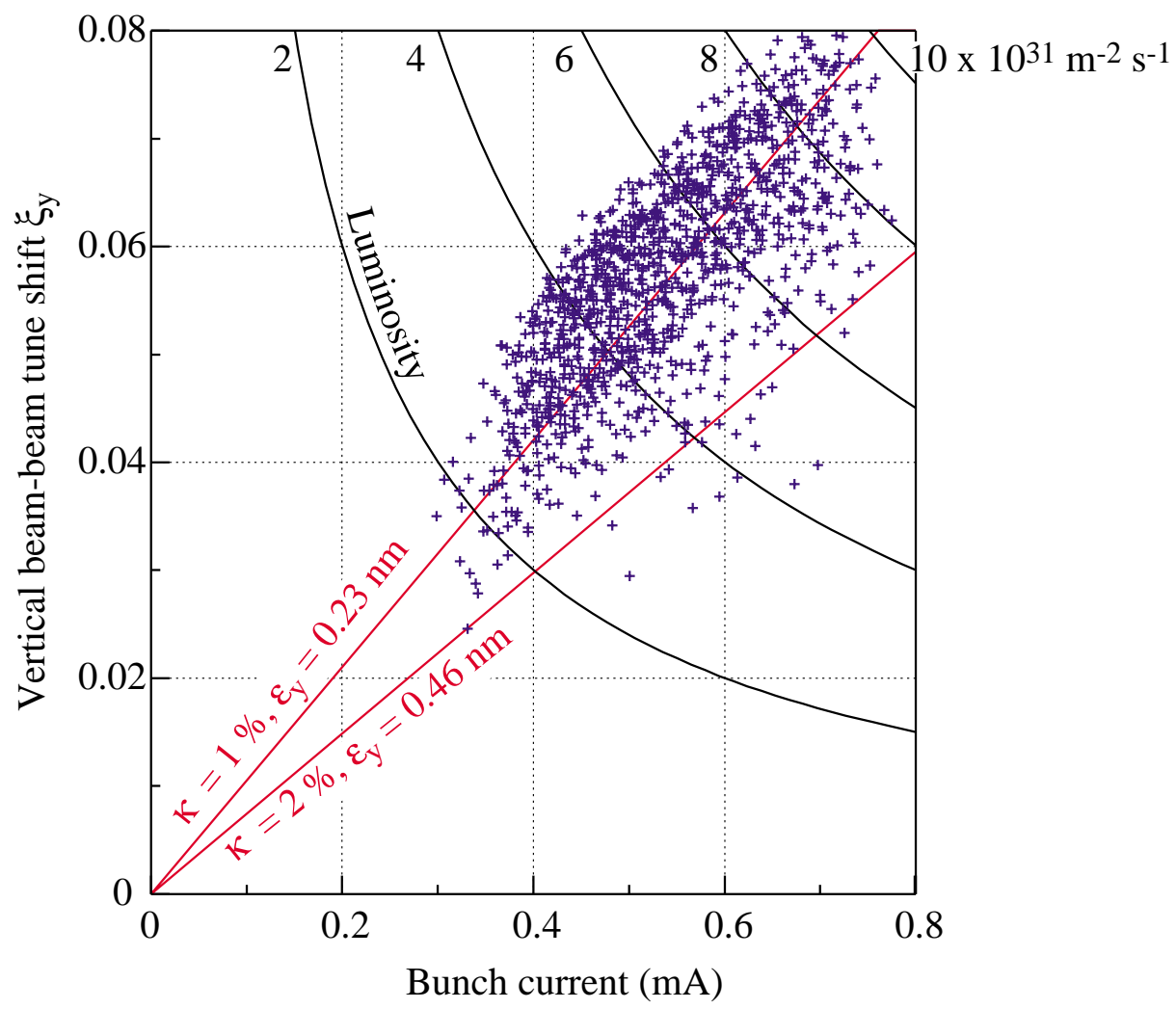

Figure 17: Dependence of $\xi_{\mathrm{y}}$ on bunch current measured in 1999 for a beam energy of $98 \mathrm{GeV}$. The betatron functions at the interaction points are $\beta_{\mathrm{x}}^{*}=1.5 \mathrm{~m}$ and $\beta_{\mathrm{y}}^{*}=5 \mathrm{~cm}$. Lines of constant luminosity and emittance are also shown. $\xi_{\mathrm{y}}$ grows almost linearly with bunch current.

with even tunes and the phase advance between the interaction points is close to an integer multiple of $2 \pi$ (typically $Q_{\mathrm{y}} / n_{\mathrm{c}}=96.18 / 4=24.045$ ). For the highest $\xi_{\mathrm{y}}$ of 0.079 in Table 3 , the actual tune shift $\delta Q_{\mathrm{y}}$ is in fact 0.051 . This number exceeds the historical maximum obtained at ADONE where $\xi_{\mathrm{y}}=0.08$ was obtained with $\delta Q_{\mathrm{y}}=0.03$ by running at the coupling resonance (round beams) [33].

It is instructive to compare the maximum $\xi_{\mathrm{y}}$ and the damping times [17] for various energies. Table 4 shows that by approximatively doubling the beam energy the parameter $k_{\mathrm{b}} \tau f_{\text {rev }}$ (which is the number of beam-beam interactions in a damping time) is decreased by a factor of 10 . The damping time $\tau$ refers to a damping partition number $J=1$.

Table 4: Maximum beam-beam tune shift $\xi_{\mathrm{y}}$, damping time $\tau$ and number of beam-beam interactions for one damping time $k_{\mathrm{b}} \tau f_{\text {rev }}$ for various beam energies at LEP.

\begin{tabular}{cccc}
\hline $\begin{array}{c}E_{\mathrm{b}} \\
(\mathrm{GeV})\end{array}$ & $\xi_{\mathrm{y}}$ & $\begin{array}{c}\tau \\
(\mathrm{msec})\end{array}$ & $k_{\mathrm{b}} \tau f_{\mathrm{rev}}$ \\
\hline 45.6 & 0.044 & 64.2 & 2880 \\
65.0 & 0.051 & 22.2 & 996 \\
97.8 & 0.079 & 6.50 & 292 \\
\hline
\end{tabular}




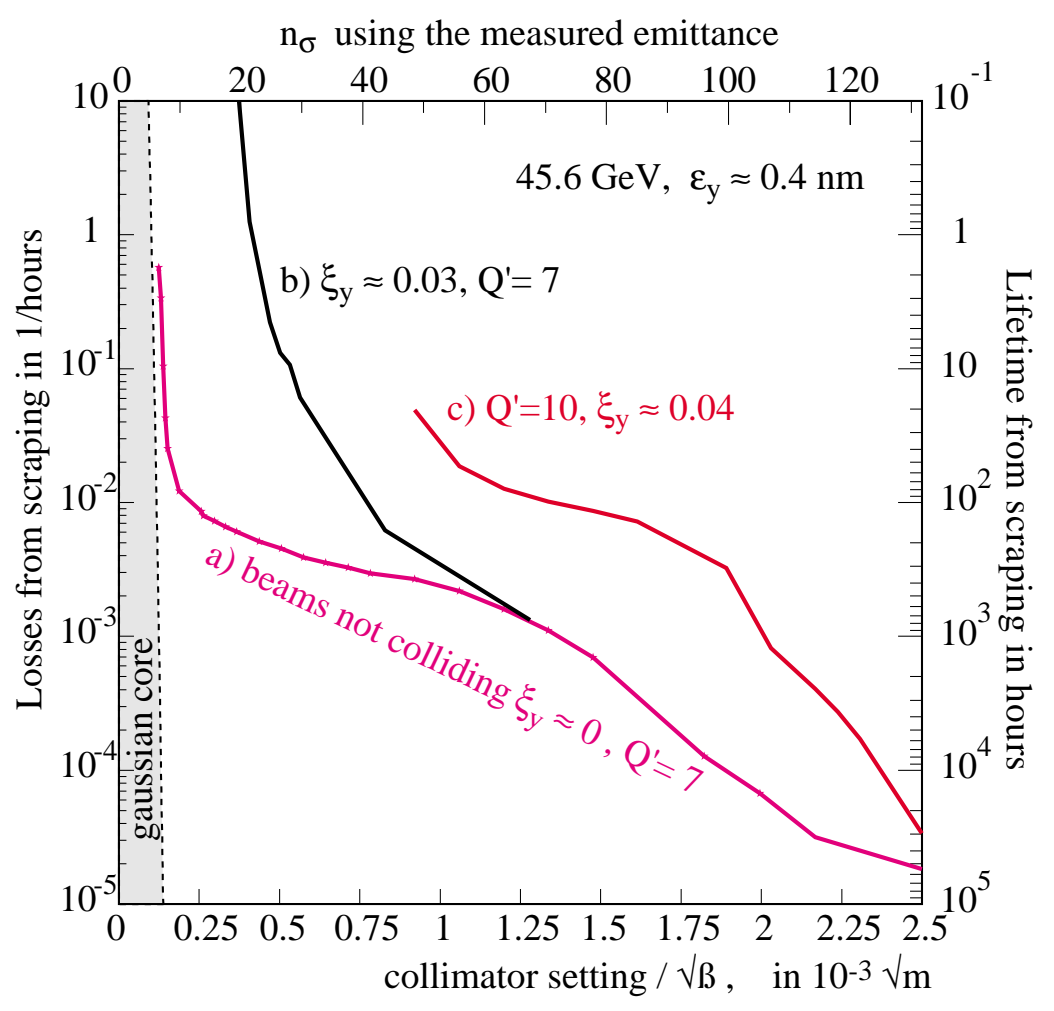

Figure 18: Beam tails in the vertical plane for $E_{\mathrm{b}}=45.6 \mathrm{GeV}$. The losses due to the scraping (vertical axis, right) and the beam lifetime $\tau$ from scraping (vertical axis, left) are shown as a function of the collimator position (lower hor. axis) and as a function of $n_{\sigma}$ (upper hor. axis). a) Without colliding beams, the core of the beam (corresponding to short lifetimes, large losses from scraping and tight collimator positions) has a Gaussian shape. There is a broad tail at large distances from the core of the bunch caused by particle scattering processes. b) and c) A significant broadening of the beam is observed with colliding beams, increasing with $\xi_{\mathrm{y}}$ and chromaticity $Q^{\prime}$.

\section{Beam tails}

To a very good approximation, the beam profiles in LEP have a Gaussian shape. The Gaussian beam core is visible with synchrotron light monitors and continuously displayed in the control room. For a beam with a Gaussian profile and an r.m.s. given by $\sigma, 95.5 \%$ of the particles are contained within $\pm 2 \sigma$. If all particles beyond $n_{\sigma} \sigma$ are removed by a solid aperture, the expected beam lifetime $\tau$ is [8] :

$$
\tau=\frac{\tau_{\mathrm{d}}}{n_{\sigma}} \exp \left(\frac{n_{\sigma}^{2}}{2}\right)
$$

where $\tau_{\mathrm{d}}$ is the damping time. For a $\tau_{\mathrm{d}}$ of $60 \mathrm{~ms}$ at $45 \mathrm{GeV}$, the lifetime is 0.09 hours at $n_{\sigma}=4.5$, 11.2 hours at $n_{\sigma}=5.5$ and 3800 hours at $n_{\sigma}=6.5$. Beam profiles have been studied quite extensively in LEP using scraping collimators and loss-monitors [34]. Beam tail measurements were also used to map the available aperture. Very good agreement with Equation (49) was found for scraping close to the beam core $\left(n_{\sigma} \leq 6\right)$, with lifetimes in the range of 0.1 to 10 hours. At larger distances from the core, corresponding to lifetimes of the order of 1000 hours, extended non-Gaussian tails were observed both planes. They can be explained to a large extent by scattering processes and off-momentum particles [35]. The importance of the tails can increase significantly at high bunch currents and high beam-beam tune shifts and cause background and 


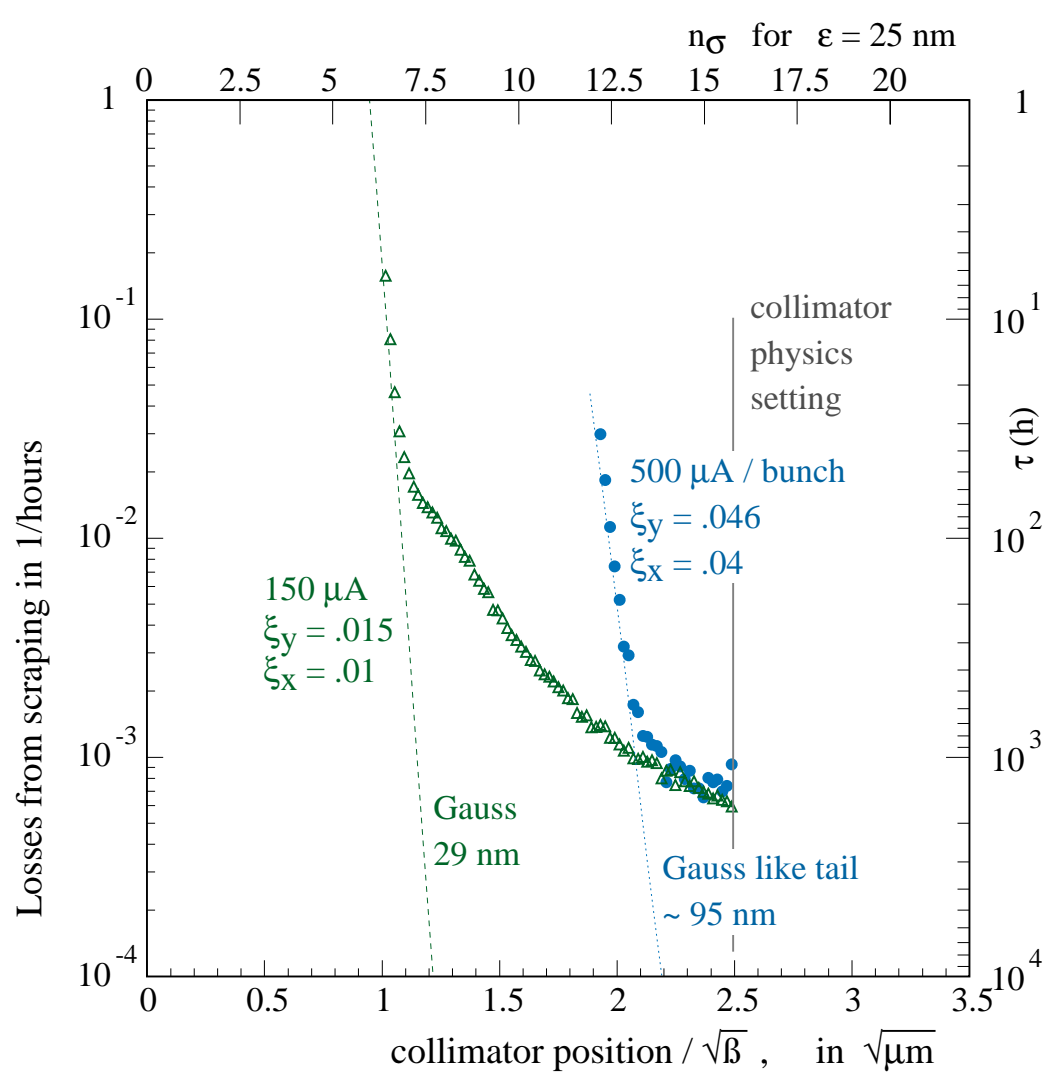

Figure 19: Observed horizontal beam tails at $65 \mathrm{GeV}$ for low and high horizontal beam-beam tune shift.

lifetime problems. This effectively limited the maximum current that could safely be collided at LEP1 to about $400 \mu \mathrm{A}$. High chromaticities were found to further enhance tails, as can be seen in Figure 18.

Figure 19 shows horizontal tail scans done at $65 \mathrm{GeV}$. A strong tail at about $12 \sigma_{\mathrm{x}}$ from the core emittance was observed for high horizontal beam-beam tune shifts which did not cause significant lifetime or background problems. It is interesting to note, that the broadened curve for high $\xi$ rises about as steeply as expected for a Gaussian beam. This can be, at least partially due to the substantial $\beta$ beating, introduced by the extra focusing effect of the beam-beam interaction.

\section{Beam lifetime}

Particles are lost from the circulating beams when the amplitude of their transverse oscillations exceeds the lateral acceptance or when their energy deviation exceeds the energy acceptance. Slow amplitude growth from higher order resonance excitation is not very important in LEP, due to the strong radiation damping. LEP runs with a sufficient over-voltage $(\sim 80$ to $160 \mathrm{MV})$ such that beams are not lost by a trip of a single RF-unit. Losses and lifetime in LEP are almost exclusively due to particle scattering processes [36] :

- Beam-gas scattering

- Compton scattering on black-body photons

- Small angle radiative Bhabha scattering in $\mathrm{e}^{+} \mathrm{e}^{-}$collisions (beam-beam bremsstrahlung) 
The scattering angles for these processes, of the order of $1 / \gamma(11 \mu \mathrm{rad}$ at $45.6 \mathrm{GeV})$, are negligible at LEP energies. The scattered particles are mainly lost because their energy deviation $\Delta E / E$ exceeds the energy acceptance of the machine which is typically $1.4 \%$.

\subsection{Beam gas scattering}

For high energy electrons or positrons, the emission of bremsstrahlung photons during the scattering of a particle on the rest gas in the vacuum chamber is an important loss mechanism. The photon (or energy loss) spectrum from bremsstrahlung is rather broad and can be approximately written as [37] :

$$
\frac{d \sigma}{d k}=\frac{A}{N_{\mathrm{A}} X_{0}} \frac{1}{k}\left(\frac{4}{3}-\frac{4}{3} k+k^{2}\right)
$$

where $k$ is the photon energy in units of the beam energy, $N_{\mathrm{A}}$ the Avogadro constant and $X_{0}$ the radiation length of the gas. The cross section scales roughly with square of the nucleus charge (through $X_{0}$ ). For $\mathrm{N}_{2}$ or $\mathrm{CO}$ gas pressures of 1 ntorr at room temperature, the gas density is $\rho=3.26 \times 10^{13}$ molecules $/ \mathrm{m}^{3}$. The scattering probability with an energy loss $\geq 1.4 \%$ is $\rho \sigma=2 \times 10^{-14} / \mathrm{m}$, and the resulting beam lifetime is 47 hours.

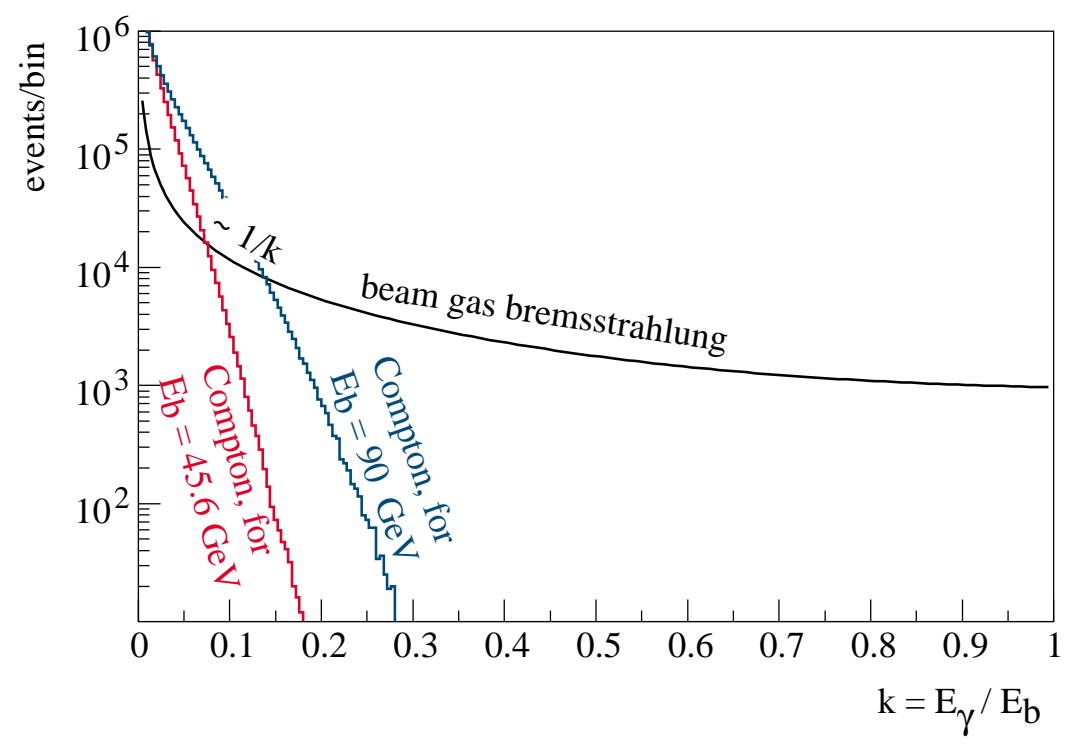

Figure 20: Comparison of the relative photon energy spectra in beam-gas scattering and Compton scattering at LEP1 and LEP2 energies.

\subsection{Compton scattering on black body radiation}

The relevance of Compton scattering of the particles on the black body radiation (thermal photons) from the vacuum chamber for high energy accelerators was first pointed out in 1987 [38]. The energy spectrum of the scattered photons has been measured in LEP and found to be in agreement with the expectations [39]. The lifetime from scattering off thermal photons is:

$$
\tau_{\mathrm{t}}=\underbrace{\frac{1}{\rho_{\gamma} c \sigma_{\mathrm{C}}}}_{26.2 h} \frac{1}{f_{\mathrm{loss}}}
$$


where $\rho_{\gamma}\left(5.329 \times 10^{14} \mathrm{~m}^{-3}\right.$ for $\left.\mathrm{T}=24^{\circ} \mathrm{C}\right)$ is the photon density, $\sigma_{\mathrm{C}}$ the Compton cross section (approximately equal 0.6652 barn) and $f_{\text {loss }}$ the fraction of the $\mathrm{e}^{ \pm}$lost after collision. The average energy of the scattered photons is about $1.1 \%$ of the beam energy at LEP1 and about $2.2 \%$ at LEP2. The energy spectra for beam-gas and Compton scattering are shown in Figure 20.

\subsection{Radiative Bhabha scattering}

The lifetime due to particles lost through radiative Bhabha scattering $\left(\mathrm{e}^{+} \mathrm{e}^{-} \rightarrow \mathrm{e}^{+} \mathrm{e}^{-} \gamma\right)$ at $n_{\mathrm{c}}$ collision points is :

$$
\tau_{\mathrm{b}}=\frac{2 r_{\mathrm{e}}}{n_{\mathrm{c}} f_{\mathrm{rev}} \sigma_{\mathrm{b}}} \frac{\beta_{\mathrm{y}}^{*}}{\gamma \xi_{\mathrm{y}}}
$$

where $\sigma_{b}$ is the total cross section for $\mathrm{e}^{+} \mathrm{e}^{-}$scattering leading to a particle loss. The particles are scattered at very low angles and this process is often called beam-beam bremsstrahlung. The cross section for scattering of free particles $[40,41]$ is $\sigma_{\mathrm{b}}=0.3 \mathrm{barn}$ at LEP energies (for an energy acceptance of $1.4 \%$ ). For particles confined in dense bunches, a cutoff parameter corresponding to the beam size or distance between particles within the bunch has to be introduced. For LEP the cross-section is then reduced to $\sigma_{\mathrm{b}} \approx 0.21$ barn and it is almost independent of the beam energy [42]. Inserting numerical values we obtain for Equation (52) :

$$
\tau_{\mathrm{b}} \approx \frac{0.44 \mathrm{~h}}{\xi_{\mathrm{y}}} \text { at } 94.5 \mathrm{GeV} \quad \text { and } \quad \tau_{\mathrm{b}} \approx \frac{1 \mathrm{~h}}{\xi_{\mathrm{y}}} \quad \text { at } 45.6 \mathrm{GeV} .
$$

The proportionality between $\xi_{\mathrm{y}}$ and the inverse lifetime in collisions has been used extensively at LEP2 as a fast measurement of the machine luminosity and of $\xi_{\mathrm{y}}$.

\subsection{Lifetime observations at LEP}

Before beams are brought into collisions, lifetimes are typically in the range of 30 (LEP2) to 50 hours (LEP1). Under good vacuum conditions, the dominant single beam loss mechanism is the process of Compton scattering on photons. When the beams are brought into collision, the lifetime decreases to typically 20 hours at LEP1 and 5 to 10 hours at LEP2. Radiative Bhabha scattering becomes then the dominant process, the lifetime component due to the $\mathrm{e}^{+} \mathrm{e}^{-}$ collisions being inversely proportional to $\xi_{\mathrm{y}}$.

Additional losses, not accounted for by scattering processes, were occasionally seen at high currents and beam-beam tune shifts. This can be explained by non Gaussian tails extending into the aperture, produced by resonance excitation in the beam-beam collisions. Such lifetime drops and background spikes were mainly observed at LEP1 energies, and generally very sensitive to minor ( 0.004) tune changes.

Figure 21 shows the evolution of beam currents and luminosity with time for a fill in LEP. The luminosity is averaged over the four LEP experiments for time intervals of 15 minutes. The lifetimes are measured with good precision in a time interval of 2 minutes. Both the beamcurrent and luminosity data shown in the Figure are expected to be precise to a few percent.

The total lifetime is obtained from the lifetime components of the different processes through:

$$
\frac{1}{\tau}=\sum_{i} \frac{1}{\tau_{i}}
$$

The lifetime predicted from the three contributions discussed here is shown in Figure $21 \mathrm{~b}$ and agrees very well with the observed lifetimes. The lifetime contributions are also summarised in Table 5. 


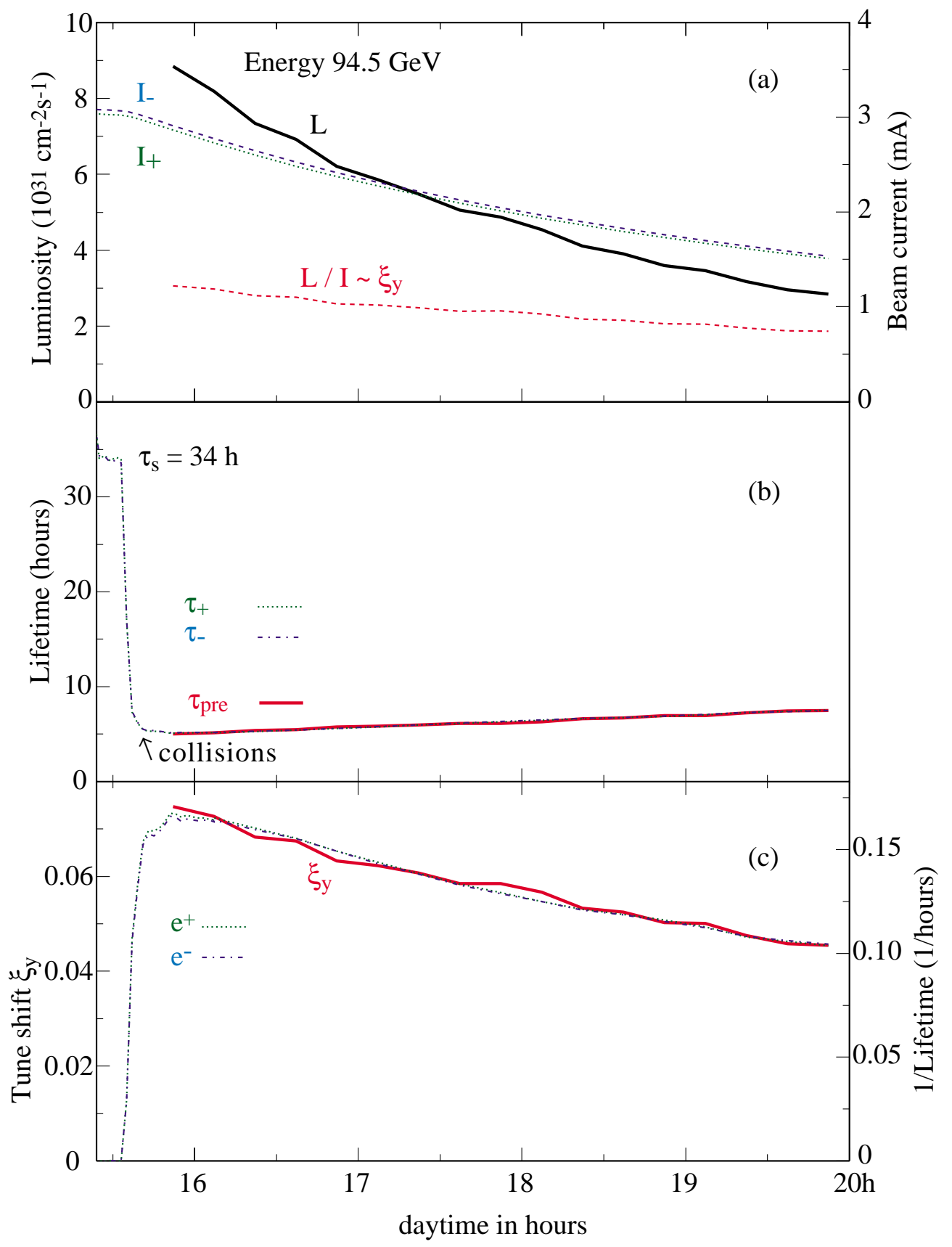

Figure 21: Evolution of some beam parameters for one LEP fill at $94.5 \mathrm{GeV}$. a) Luminosity $\mathcal{L}$ and beam currents $I$. The ratio $\mathcal{L} / I$ which is proportional to the beam-beam tune shift $\xi_{\mathrm{y}}$ is also shown. b) Observed positron $\tau^{+}$and electron $\tau^{-}$lifetimes and the predicted lifetime in collisions $\tau_{\text {pre. }}$. The lifetime drops from 34 hours to about 5 hours when the beams are brought into collision. c) The vertical beam-beam tune shift parameter $\xi_{\mathrm{y}}$, extracted from the measured luminosity, and the inverse lifetime (single beam lifetime subtracted) 
Table 5: Contributions to the lifetime for the beginning of the LEP fill shown in Figure 21

\begin{tabular}{lc}
\hline Process & $\tau$ (hours) \\
\hline Compton scattering & 60 \\
Beam-gas scattering (pressure of 0.6 ntorr) & 80 \\
Radiative Bhabha scattering $\left(\xi_{\mathrm{y}}=0.075\right)$ & 5.8 \\
Total & 5.0 \\
\hline
\end{tabular}

\section{Background sources and shielding}

The main background sources for the LEP detectors are photons from synchrotron radiation and off-momentum electrons (and positrons) from beam-gas and Compton scattering on black body radiation.

\subsection{Synchrotron radiation background}

The energy spectrum of the synchrotron radiation photons radiated by a high energy electron travelling in a circular orbit of radius $\rho$ is characterised by the critical energy :

$$
E_{\mathrm{c}}=\frac{3}{2} \hbar c \frac{\gamma^{3}}{\rho}=70 \mathrm{keV} \text { at } 45.6 \mathrm{GeV} \quad \text { and } \quad 733 \mathrm{keV} \text { at } 100 \mathrm{GeV}
$$

The number of photons radiated by an electron per turn is ( $\alpha=e^{2} / 4 \pi \epsilon_{0} \hbar c$ is the fine-structure constant):

$$
N_{\gamma}=\frac{5 \pi \alpha \gamma}{\sqrt{3}}=5906 \text { at } 45.6 \mathrm{GeV} \text { and } 12951 \text { at } 100 \mathrm{GeV}
$$

The amount of synchrotron radiation in LEP is huge, particularly at LEP2 energies : about $6 \times 10^{20}$ photons are emitted per second and a power of $18 \mathrm{MW}$ is lost to synchrotron radiation. The experiments have to be very well screened with a sophisticated collimation system [43]. The typical layout of the collimators in a straight section, where only scattered synchrotron light reaches the detectors, is shown in Figure 22. The synchrotron radiation spectrum is broad and photons down to about $20 \mathrm{keV}$ can leave the beam pipe. The lower energy X-ray radiation can undergo low angle (multiple) reflection [44]. The strong radiation from the main dipoles is intercepted close to the arcs, with collimators located between 100 and $220 \mathrm{~m}$ away from the interaction point, before the photons are scattered at low angle towards the experiments. To reduce the radiation shining into the straight sections further, the first dipoles in the arcs have only $10 \%$ of the field of the normal arc dipoles.

Local masks were installed about $2.4 \mathrm{~m}$ from the interaction points to improve the shielding of the experiments from the increased synchrotron radiation at LEP2 [45, 46]. The collimators and masks close to the interaction point are also a source of scattered background particles. The surface material and surface angle of the masks were optimized to minimize the scattering towards the experiment : the masks are made of tungsten and the surface is coated with silver and copper layers to reduce fluorescence photons.

The background photons observed in the detector originate mainly from synchrotron radiation in the last quadrupoles and are backscattered into the experiment from local collimators. The bunch crossing rate in LEP is about $45 \mathrm{kHz}$ and typically only a few background photons were recorded per bunch crossing in the large wire chambers of the LEP detectors. There was no problem with detector occupancy, but the currents drawn in the gas-chambers were reported to be not too far from the tolerable limit. 


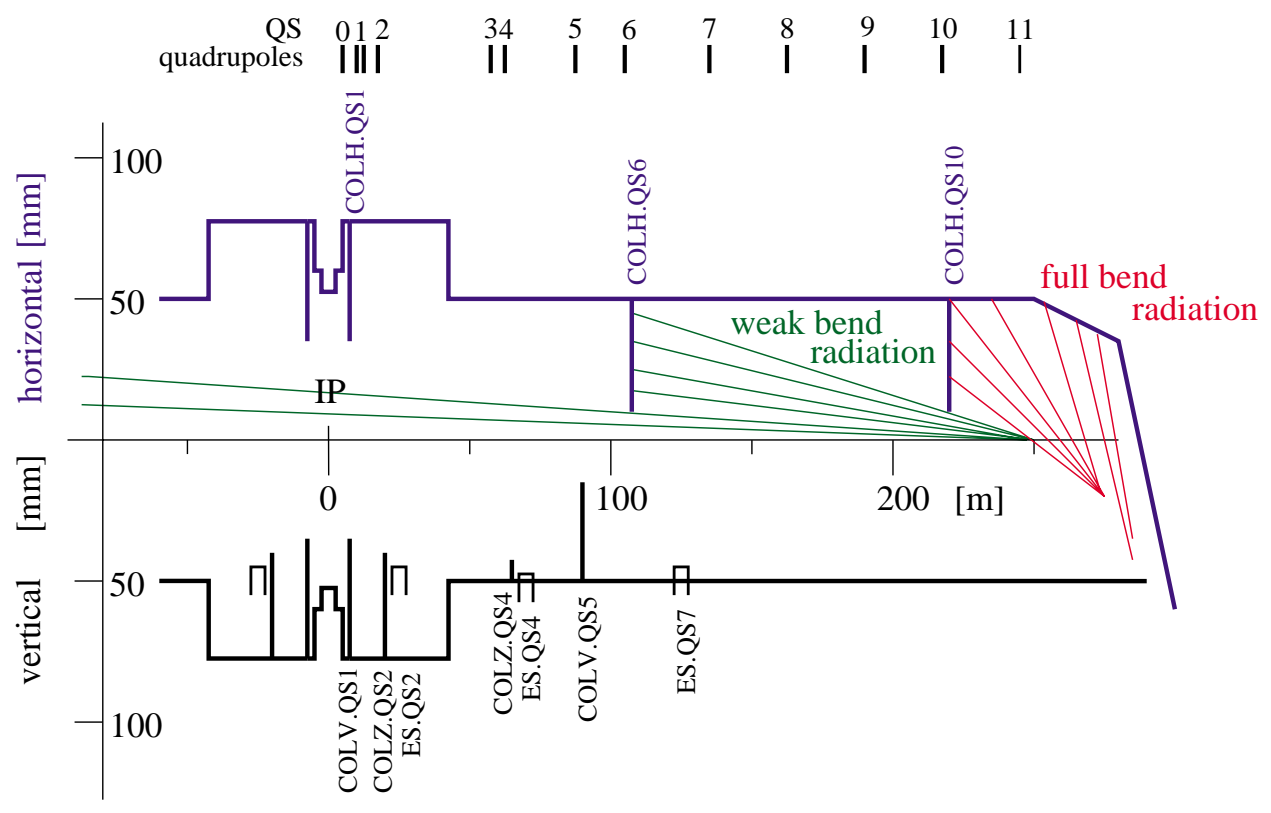

Figure 22: Schematic layout of a straight section at an interaction point (IP) of LEP in the horizontal (top) and vertical (bottom) planes. Shown are the locations of the quadrupoles (QS), electrostatic separators (ES) and collimators (COLH, COLV, COLZ). The solid lines mark the inner vacuum chamber radii.

\subsection{Off-momentum background}

Off-momentum particles, which have lost a significant fraction of their energy, are produced by inelastic scattering processes. A first source of off-momentum particles is the emission of bremsstrahlung during a scattering of beam particles on rest gas in the vacuum chamber. A second source is due to Compton scattering of particles on the thermal photons from the blackbody radiation. These processes are also of importance for the beam lifetime and were discussed in the preceding chapter. Simulations of the scattering processes and tracking trough the lattice provided a quantitative prediction and understanding of the kinematics of the off-momentum background [43].

The maximum energy acceptance in the machine LEP is about $3 \%$, but only particles that remain captured by the RF system (inside the RF-bucket) are stable over many turns. For LEP the energy acceptance from the RF over-voltage is typically $\pm 1.4 \%$ (see also Chapter 1 , Equation(10)). Particles with an energy loss between about $1.4 \%$ and $3 \%$ can still travel through several arcs. They are over-focused by the last quadrupoles around the experiments and and may be lost within $10 \mathrm{~m}$ of the interaction region. Dedicated off-momentum collimators were installed near the end of the arcs around the experimental straight sections to intercept such particles. Otherwise, they would mainly hit the collimators next to the experiments (about $8.5 \mathrm{~m}$ from the IP) at a rate of about $10 \mathrm{kHz}$. These collimators in LEP are instrumented and used for luminosity optimization.

Particles must have lost over $10 \%$ of their energy to hit the experiments directly, mainly in the detectors used for the luminosity measurement at low angles. Such particles are produced locally by beam-gas bremsstrahlung and under good vacuum conditions (pressures about 1 ntorr CO), the LEP detectors are hit by off-momentum particles at a rate of $\sim 100 \mathrm{~Hz}$. This is small $\left(2.2 \times 10^{-3}\right)$ compared to the bunch crossing rate of $45 \mathrm{kHz}$. The probability for random coincidences of off-momentum background particles is therefore rather low. This has been very 
useful for the precise luminosity determination by the experiments.

The LEP experiments are equipped with fast pin-diode radiation monitors, connected to the beam-dump system to prevent major beam loss with possible damage into the detectors.

There has always been a substantial effort in LEP to control and minimize the background, resulting in usually very clean running conditions for the experiments. One should however realize, that this generally implies a compromise in luminosity performance. Background considerations have in fact set limits on the lowest practical $\beta^{*}$ and on the maximum current in collisions at $45 \mathrm{GeV}$.

\section{Luminosity optimization}

The primary aim of LEP is the delivery of luminosity to the experiments and the key measure of success is the delivered integrated luminosity. Once a given beam current has been successfully accumulated and accelerated to the relevant physics energy, a lot of effort is put into optimizing the beam sizes and collisions.

The luminosity of two colliding Gaussian beams may be expressed as (see also Equation 15):

$$
\mathcal{L}=\frac{N_{\mathrm{b}+} N_{\mathrm{b}-} f_{\mathrm{rev}} k_{\mathrm{b}} \exp \left\{-\frac{\left(\overline{\mathrm{x}}_{-}-\overline{\mathrm{x}}_{+}\right)^{2}}{2\left(\sigma_{\mathrm{x}+}^{2}+\sigma_{\mathrm{x}-}^{2}\right)}-\frac{\left(\bar{y}_{-}-\bar{y}_{+}\right)^{2}}{2\left(\sigma_{\mathrm{y}+}^{2}+\sigma_{\mathrm{y}-}^{2}\right)}\right\}}{2 \pi \sqrt{\left(\sigma_{\mathrm{x}+}^{2}+\sigma_{\mathrm{x}-}^{2}\right)\left(\sigma_{\mathrm{y}+}^{2}+\sigma_{\mathrm{y}-}^{2}\right)}} .
$$

By inspection of the above formula one can see why luminosity optimization at LEP essentially consists of meeting the following challenges:

- ensuring that the beams collide head on at the interaction points,

- reducing the vertical emittance by good correction of coupling and residual vertical dispersion,

- adjusting optics parameters to reduce both the horizontal and vertical beam size at the interaction points.

As somewhat of a sideline issue, time is also spent attempting to address possible sources of luminosity imbalance between the experiments.

Over the years LEP has essentially operated in two regimes: the first was on or around the $Z^{0}$ peak, the second at energies above the $W^{ \pm}$threshold. In the former regime performance was constrained by the beam-beam effect.

At higher energies increased damping meant the effects of beam-beam were less of a problem, and optimization was able to concentrate on reduction of the main contributions to the beam sizes enumerated below.

\subsection{Head-on collisions}

The degree of the overlap of the counter-rotating beams is clearly of importance. In an insertion one would expect the two counter rotating beams to follow more-or-less the same orbits in the horizontal and vertical planes, and for the collision point to be given, without adjustment to within a few microns. The beam sizes at the interaction points (see Table 3) are typically of the order of 200 microns horizontally, and 3 microns vertically. Thus any mis-crossing will have considerably more impact in the vertical plane. This consideration is exacerbated by the presence of vertical separation "bumps" in the odd points. These bumps are invariably not perfectly closed leading to slightly different vertical orbit for electrons and positrons around the ring; these can manifest themselves as sizable mis-crossings in the vertical plane. 
The maximization of the overlap in the vertical plane, or equivalently, ensuring head-on collisions, was done by two standard techniques: luminosity scans, and beam-beam deflection scans.

In both, the crossing point of the two beams is adjusted by the use of closed orbit bumps generated by electrostatic separators. Note that the electron and positron beams move in opposite directions at the interaction point. The aim is to find the settings of these bumps which give head-on collisions and thus maximize the luminosity. The beams are incrementally moved across each other at a given interaction point using the closed bumps. Generators used in the scans to produce the voltage on the separators have a fine resolution (the so-called vernier generators) and scans have become known generically as "vernier scans".

\subsubsection{Luminosit y scans}

As described above the beams are moved across each other in steps; typically the beams are initially separated by $20 \mu \mathrm{m}$ and then bumped in 2 to $3 \mu \mathrm{m}$ steps across each other. At each step a luminosity measurement, or the average of several measurements, is taken from the LEP luminosity monitors. The optimum setting of the separators is given by the maximum extracted from a Gaussian fit to the scan results.

Such scans take about 5 minutes and are limited by the time need to accumulate sufficient luminosity statistics. Typical results from bunch train running are shown in Figure 23.

Besides collision offsets vernier scans were also used to measure the difference in vertical dispersion at the IPs of the two beams. This measurement became particularly important during attempts to precisely determine the beam energies when running with bunch trains [47].

\subsubsection{B eam-beam scans}

At high energy the luminosity monitors which use Bhabha scattered leptons as the observable become less efficient because of lower angular acceptance.

The mutual electro-magnetic deflections of the two interacting beams produced by the the beam-beam force are measured by extrapolating in from two sets of two orbit position monitors situated either side of the interaction point. It is clear that if the beams collide headon one would expect zero deflection. Further, the equation for the variation of the beam-beam deflection with the transverse offset is well known. By fitting the measured results to this equation one is able to extract the optimum setting of the vernier generators and at the same time the beam sizes at the interaction points [48]. The results from a typical scan are shown in Figure 24.

\subsection{Optical parameters at the interaction points}

Having successfully ensured head-on collisions at the interaction points, the next challenge is the reduction of the beam-sizes at said interaction points. Optimization in this respect can be neatly divided into two: firstly the adjustment of local optical parameters to reduced the beam size at the IPs directly, and secondly the reduction of the beam emittance, essentially a global optimization. Consider first the adjustment of local optical parameters. 


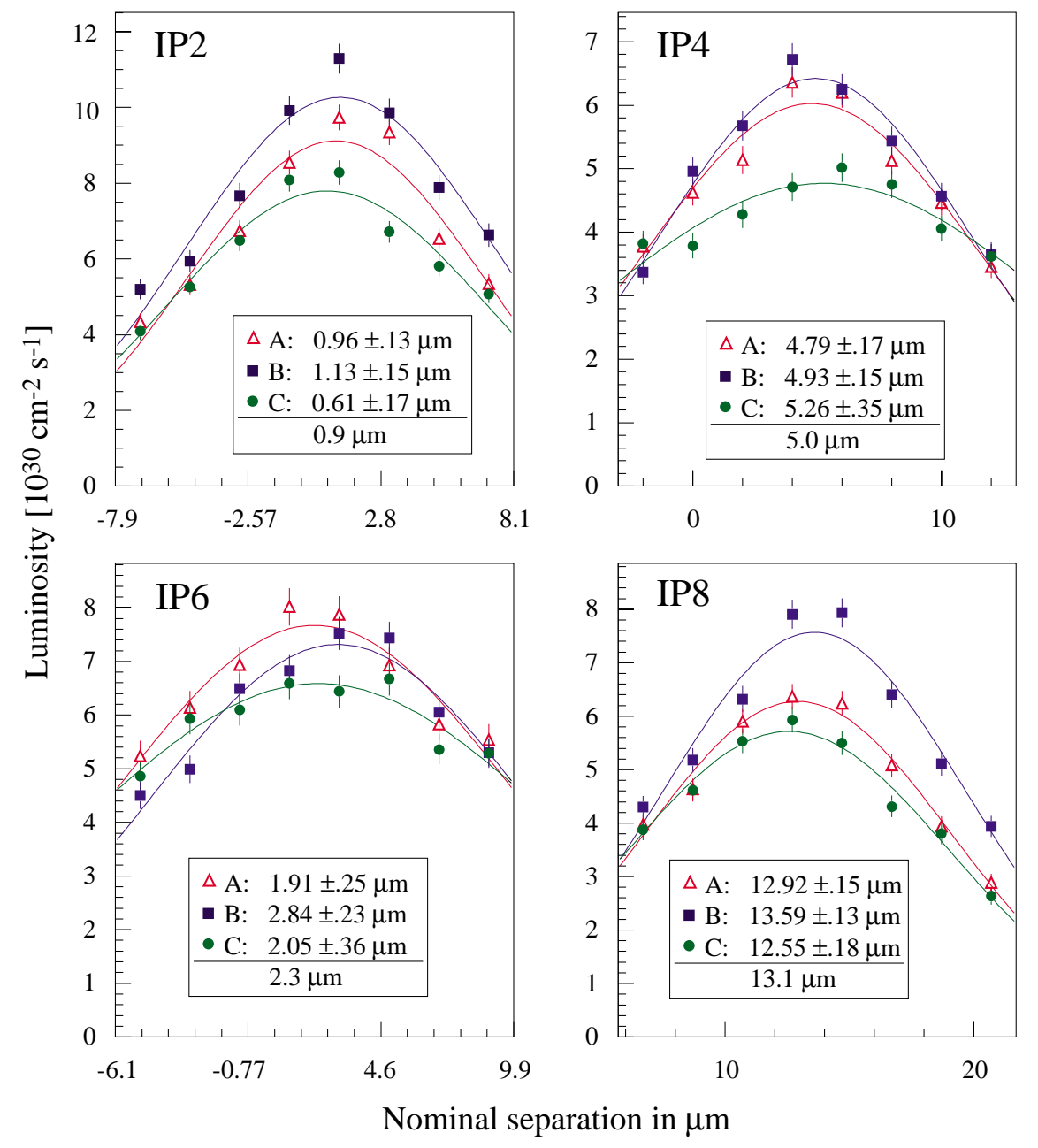

Figure 23: Luminosity as a function of the beam separation at the four LEP IPs. The offsets are separately extracted by applying a Gaussian fit to the luminosity data of each of the three bunch train families. 


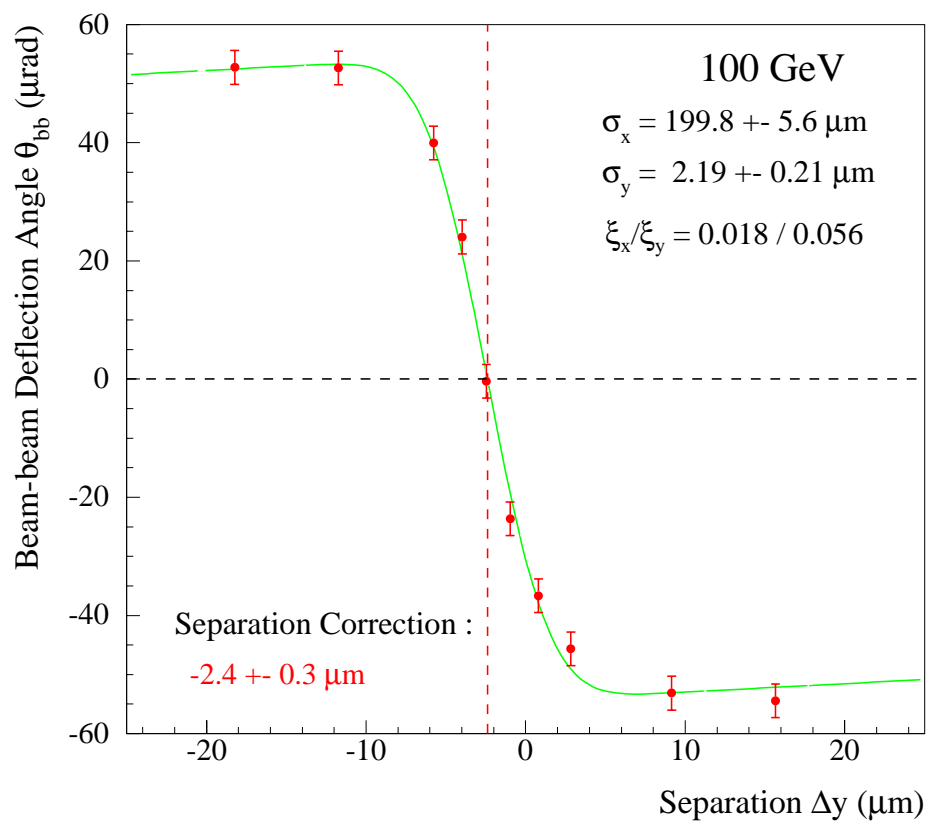

Figure 24: Deflection angle of the beams due to their mutual interaction as a function of the beam separation. The deflection vanishes when the beams collide head-on. Both transverse beam sizes can be extracted from the data.

\subsubsection{Reduction of $\beta_{\mathrm{y}}^{*}$}

For head-on collisions and equal beam sizes and currents for both beams, the luminosity per interaction region is:

$$
\mathcal{L}=\frac{N_{\mathrm{b}}^{2} f_{\mathrm{rev}} k_{\mathrm{b}}}{4 \pi \sigma_{\mathrm{x}} \sigma_{\mathrm{y}}}=\frac{N_{\mathrm{b}}^{2} f_{\mathrm{rev}} k_{\mathrm{b}}}{4 \pi \sqrt{\epsilon_{\mathrm{x}} \beta_{\mathrm{x}}^{*}} \sqrt{\epsilon_{\mathrm{y}} \beta_{\mathrm{y}}^{*}}}=\frac{\gamma N_{\mathrm{b}} f_{\mathrm{rev}} k_{\mathrm{b}} \xi_{\mathrm{y}}}{2 r_{\mathrm{e}} \beta_{\mathrm{y}}^{*}} .
$$

The advantages of reducing $\beta_{\mathrm{y}}^{*}$ are fairly obvious : a reduction from $\beta_{\mathrm{y} 1}^{*}$ to $\beta_{\mathrm{y} 2}^{*}$ would give an increase in luminosity of $\sqrt{\beta_{\mathrm{y} 1}^{*} / \beta_{\mathrm{y} 2}^{*}}$, if one is not already at the beam-beam limit and if everything else were to remain equal.

The key point to made at this juncture is that to enjoy the benefit of reducing $\beta_{\mathrm{y}}^{*}$, the vertical emittance has to be (re)established at its minimum value. As described below a small vertical emittance is achieved by a careful optimization of the vertical and horizontal crossings, the vertical orbit, and coupling. Because of this it is often very difficult to observe directly an improvement in the luminosity following the adjustment of $\beta_{\mathrm{y}}^{*}$.

Decreasing $\beta_{\mathrm{y}}^{*}$ necessarily increases $\beta_{\mathrm{y}}$ at the super-conducting quadrupoles. The effect of any errors in these magnets will be magnified and be reflected in increased $\beta$ beating. The increase in $\beta_{\mathrm{y}}$ at the super-conducting quadrupoles increases the beam sizes in these magnets with implications for the experiments' background. In short although the possibility of squeezing beyond $5 \mathrm{~cm}$ was investigated at various times and indeed LEP2 did run for a time with $\beta_{\mathrm{y}}^{*}$ $=4 \mathrm{~cm}$ no manifest gains were apparent and $\beta_{\mathrm{y}}^{*}=5 \mathrm{~cm}$ remained nominal.

\subsubsection{Reduction of $\beta_{\mathrm{x}}^{*}$ and $J_{\varepsilon}$}

As can be seen from equation 56 the reduction of $\beta_{\mathrm{x}}^{*}$ is of interest because: 


$$
\mathcal{L} \propto \frac{1}{\sqrt{\beta_{\mathrm{x}}^{*}}}
$$

and naturally enough it was reduced as far as possible at high energies when LEP was not running at the beam-beam limit. The acceptable minimum is limited by

- background considerations due to increased beam size in the last quadrupoles before the interaction point,

- and the possibility of reduced dynamic aperture. In strong focusing lattices such as $102^{\circ} / 90^{\circ}$ the strength of the sextupoles are already large and this can have two detrimental effects: it creates large anharmonicities (tune dependence on amplitude) and excites higher order resonances. These two effects may reduce the usable dynamic aperture. The required increase of the sextupole strengths when reducing $\beta_{\mathrm{x}}^{*}$ serves to reduce the dynamic aperture even further.

At LEP2 the reduction of $\beta_{\mathrm{x}}^{*}$ from 2.0 to $1.5 \mathrm{~m}$ was performed without problems with a further squeeze to $1.25 \mathrm{~m}$ introduced judiciously [49]. In fact, concerns about the experiments' backgrounds, particularly from synchrotron radiation, led to a $\beta_{\mathrm{x}}^{*}=1.5 \mathrm{~m}$. being used as standard.

The dependence of the horizontal emittance on the horizontal damping number $J_{\mathrm{x}}$ is given in Equation (29). Thus via Equation (15) the effect on the luminosity of reducing the horizontal emittance by increasing the horizontal damping partition number is:

$$
\mathcal{L} \propto \frac{1}{\sqrt{J_{\mathrm{x}}}} .
$$

Here the assumption is made that the vertical emittance remains unchanged when the horizontal emittance is adjusted. This will be true is the coupling is well-corrected. An RF frequency shift of between $\Delta f_{\mathrm{RF}}=+100 \mathrm{~Hz}$ and $\Delta f_{\mathrm{RF}}=+140 \mathrm{~Hz}$ was routinely used at LEP2 changing $J_{\mathrm{x}}$ from a nominal 1 to between 1.57 and 1.80 for the $102^{\circ} / 90^{\circ}$ optics. The vertical beam size was observed to remain more-or-less constant as $J_{\mathrm{x}}$ was changed and increase in luminosity of approximately 30\% was clearly observed over an extended period [49].

\subsection{Beam emittance}

Having reduced $\beta_{\mathrm{x}}^{*}$ and increased $J_{\mathrm{x}}$ the horizontal beam size is essentially given. The principal means of increasing the performance further becomes the reduction of the vertical emittance. It is informative to attempt to quantify the contributions to the vertical emittance i.e.:

- opening angle of synchrotron radiation,

- dispersion at the interaction point,

- residual vertical dispersion around the ring,

- coupling,

- beam-beam.

Addressing each of these in turn:

\subsubsection{Finite emission angle of synchrotron radiation}

The contribution of the finite emission angle of synchrotron radiation is well-established [9]. For LEP at $91.5 \mathrm{GeV}$ the contribution is totally negligible and is of the order $10^{-6} \mathrm{~nm}$. 


\subsubsection{D ispersion at the IP}

The beam size at the interaction point may be expressed:

$$
\sigma_{\mathrm{y}}^{*}=\sqrt{\epsilon_{\mathrm{y}} \beta_{\mathrm{y}}^{*}+\left(D_{\mathrm{y}}^{*} \frac{\sigma_{\mathrm{e}}}{E_{\mathrm{b}}}\right)^{2}}
$$

where $D_{\mathrm{y}}^{*}$ the dispersion at the interaction point.

The value of the vertical dispersion at an IP is small ( $2 \mathrm{~mm}$ or less) and it was notoriously difficult to measure and in fact in the history of LEP no reliable method was developed. By scaling the r.m.s dispersion around the ring with the square root ratio of average $\beta$ values to that at the IP one gets estimate an estimate of around $0.75 \mathrm{~mm}$ which is compatible with measurements of the difference in dispersion between the two beams [47].

Taking a natural beam size of $3 \mu \mathrm{m}$ and a $0.75 \mathrm{~mm}$ dispersion one gets an effective beam size of around $3.3 \mu \mathrm{m}$ and a luminosity drop of some $10 \%$, not at all insignificant.

The increase in beam size because of local dispersion at a given interaction point clearly reduces the luminosity (and beam-beam tune shift) at that point. Besides reducing the overall performance this can also lead to luminosity imbalance between the experiments.

\subsubsection{Coupling}

An important parameter to be kept under strict control is the betatron coupling between the transverse oscillations in the horizontal and vertical planes. These oscillations can be coupled by the magnetic fields of, for example, skew-quadrupoles and solenoids. The presence of strong coupling can induce optics distortions, $\beta$ beating etc. and can perturb and confuse the checking and control of the optics. At LEP, coupling control is particularly important because of its potential impact on the vertical emittance.

One way of measuring the coupling, and the one usually used in LEP, is the so-called "Closest Tune Approach". In this method the tunes are swept across each other by varying appropriate quadrupoles, for example, $Q_{\mathrm{x}}$ is reduced and $Q_{\mathrm{y}}$ increased in the vicinity of the main coupling resonance. In the presence of coupling the measurements reveal a stop gap visible in Fgure 25 in which the horizontal and vertical tunes become indistinguishable. The width of this stop gap is a estimate of the strength of the coupling existing in the machine [21].

The contribution of coupling to the vertical beam size can be usefully expressed in terms of the coupling ratio:

$$
\frac{\epsilon_{\mathrm{y}}}{\epsilon_{\mathrm{x}}}=\frac{\left(\frac{\left|C^{-}\right|}{2 \Delta}\right)^{2}}{\left(\frac{\left|C^{-}\right|}{2 \Delta}\right)^{2}+0.5} .
$$

Here $\Delta$ is the distance between the tunes and thus the distance to the main coupling resonance. $C^{-}$is the difference coupling coefficient which is measured directly via the closest tune approach.

If the coupling is properly corrected, as was usual, with a closest approach of, say, less than 0.003 the contribution to the vertical emittance should be below $0.01 \mathrm{~nm}$.

\subsubsection{Residual vertical dispersion}

The contribution to the vertical emittance from residual vertical dispersion is given by [9] :

$$
\Delta \epsilon_{\mathrm{y}}=c_{\mathrm{q}} \gamma^{2} \frac{\left\langle\mathcal{H}_{\mathrm{y}} /\left|\rho^{3}\right|\right\rangle_{\mathrm{s}}}{J_{\mathrm{y}}\left\langle\rho^{2}\right\rangle_{\mathrm{s}}}
$$




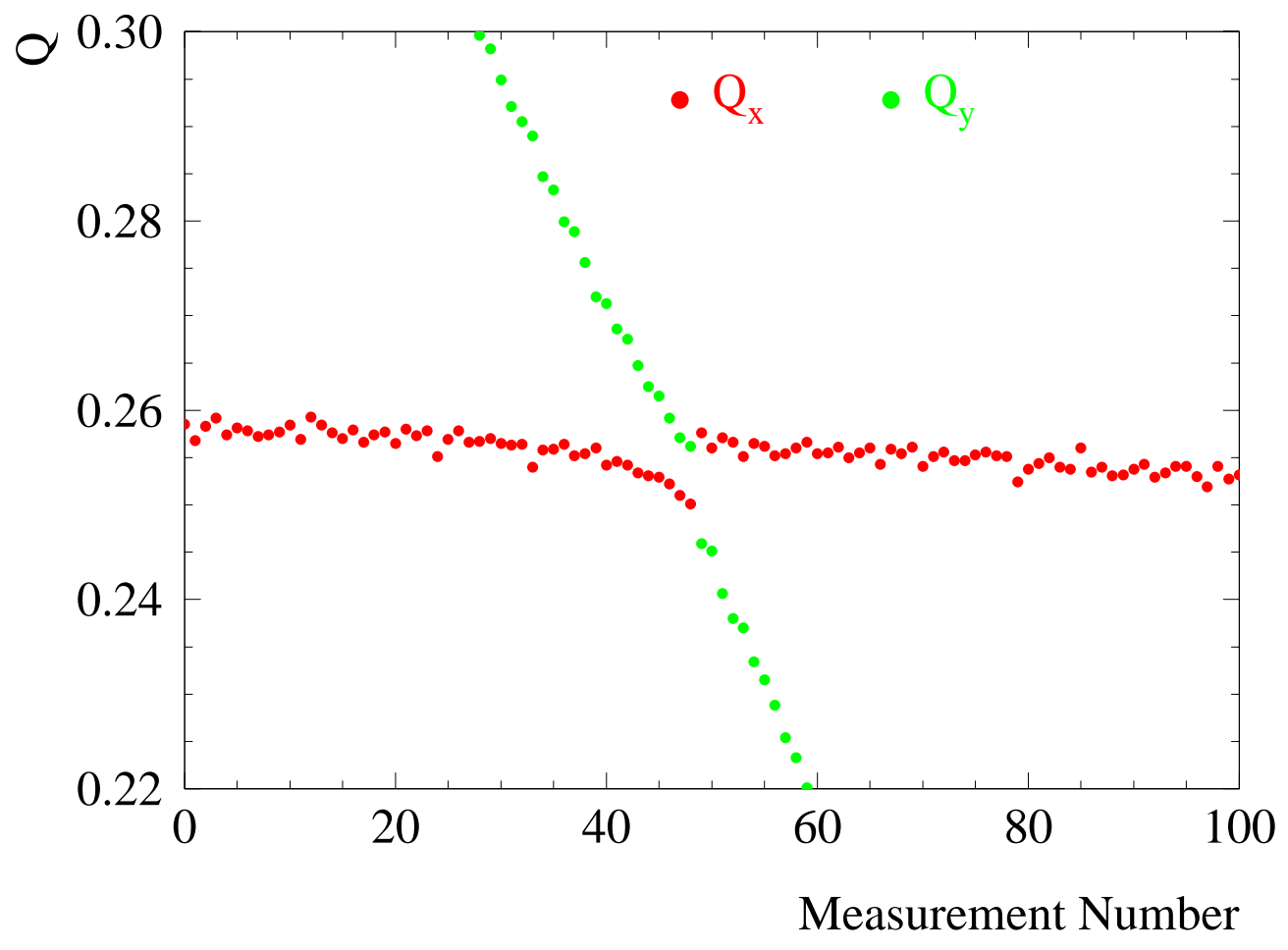

Figure 25: Evolution of the horizontal and vertical tunes during a closest tune approach measurement. In this example the coupling correction is poor and a clear stop gap is visible when the two tunes cross.

where $\mathcal{H}_{\mathrm{y}}$ is the vertical dispersion invariant :

$$
\mathcal{H}_{\mathrm{y}}=\left\langle\beta_{\mathrm{y}} D_{\mathrm{y}}^{\prime 2}+2 \alpha_{\mathrm{y}} D_{\mathrm{y}} D_{\mathrm{y}}^{\prime}+\gamma_{\mathrm{y}} D_{\mathrm{y}}^{2}\right\rangle_{\mathrm{s}}
$$

and \langle\rangle$_{\mathrm{s}}$ designates an integration over the complete ring. $\alpha_{\mathrm{y}}$ and $\gamma_{\mathrm{y}}$ are related to $\beta_{\mathrm{y}}$ and its derivative with respect to the longitudinal coordinate $s$ :

$$
\alpha_{\mathrm{y}}=-\frac{1}{2} \frac{\mathrm{d} \beta_{\mathrm{y}}}{\mathrm{d} s} \quad \text { and } \quad \gamma_{\mathrm{y}}=\frac{1+\alpha_{\mathrm{y}}^{2}}{\beta_{\mathrm{y}}}
$$

while $D_{\mathrm{y}}^{\prime}$ is the derivative of the dispersion :

$$
D_{\mathrm{y}}^{\prime}=\frac{\mathrm{d} D_{\mathrm{y}}}{\mathrm{d} s} .
$$

Typically a $5 \mathrm{~cm}$ r.m.s vertical dispersion will generate a vertical beam emittance of 0.2 to $0.3 \mathrm{~nm}$, a major contribution and a clear indication of the importance of residual vertical dispersion to performance.

\subsubsection{Dispersion free st eering}

If the coupling is properly corrected, the main contribution to the vertical emittance is the residual vertical dispersion and at LEP2 the battle for high luminosity became essentially an attack on this source. For a long time a process of iteration coupled with a pragmatic search was used to establish a so-called "golden orbit". This orbit, by virtue of low vertical dispersion, was selected by its luminosity performance. 
The development of dispersion free steering (DFS) [50] allowed a more predictive approach. This technique uses an orbit measurement and a dispersion measurement to minimize both the r.m.s orbit displacement and the r.m.s dispersion. Using a Singular Value Decomposition algorithm [51] for matrix inversion it is also possible to minimize the orbit corrector kicks at the same time. DFS was used successfully in the 1999 LEP run to establish high-performance golden orbits more rapidly and deterministically. The r.m.s vertical dispersion was reduced down to $1.5 \mathrm{~cm}$, compared to $5 \mathrm{~cm}$ in earlier years.

\subsection{Luminosity equalisation}

Although the main drive within operations is towards maximizing the instantaneous luminosity and in the long run the total integrated luminosity delivered to the experiments, some care is taken to ensure that the four different experiments receive more-or-less the same luminosity over a period of time (within something like a $\pm 5 \%$ range) [52].

Luminosity differences between the experiments are indicative of underlying asymmetries and in most cases reducing the differences simultaneously increases the overall performance.

\subsubsection{Measurement and correction of the bet at $r$ on functions}

Some of the most basic optics parameters are the $\beta$ values at the interaction points. The standard method for measuring these is via the variation of the $\beta$ function in a drift space $(\beta(s) \simeq$ $\left.s^{2} / \beta_{\text {min }}\right)$. For example, in the vertical plane, the $\beta_{\mathrm{y}}$ at the super-conducting quadrupoles left and right of the IP is calculated from the tune shift produced by a small gradient change on these magnets.

Having measured the $\beta_{\mathrm{y}}^{*} \mathrm{~s}$ at each of the IPs, a simulation program is used to calculate the requisite correction using only the super-conducting quadrupoles, which are assumed to be the main source of error. Measurements before and after correction show the $\beta$ beating to be significantly reduced, supporting the use of the above assumption.

\subsubsection{Waist scans}

The $\beta$ function at an interaction point varies according to the simple law given above. Clearly if the minimum of the $\beta$ function is displaced from the actual interaction point then degradation of performance will result. The waist can be moved by asymmetrically varying the strength of the super-conducting quadrupoles and scanning against luminosity performance. Although used occasionally to eliminate the possibility of a displaced minimum, waist adjustments have never been necessary during LEP operation.

\subsubsection{Dispersion at the collision points}

Varying vertical dispersion at the IPs can lead to different beam sizes at the IPs and thus luminosity variations.

As mentioned above one of the essential problems here is the difficulty in measuring the $D_{\mathrm{y}}$ at the interaction point. (Attempts have been made to fit dispersion measurements through the insertions but these are essentially limited by the orbit measurement system.) There is clear evidence that observed luminosity imbalance is at least partially due to varying amounts of dispersions at the IPs. Different vertical golden orbits with different vertical dispersion have been noted to make a considerable difference to problematic imbalances. 


\subsubsection{B etatron function perturbations due to beam-beam ef fect $s$}

Adjustment of $\beta^{*} \mathrm{~s}$ is performed either with a single beam or separated beams. The beam-beam effect produces a large tune spread among the particles in the beam makes it very difficult to get an accurate measure of the tune with colliding beams. In collision the beam-beam force can strongly perturbed the optics, generate beta beating and considerably reduce the effective $\beta^{*}$ at the IPs. When combined with phase errors around the machines this can led to different changes at different IPs and thus introduce a luminosity imbalance. This possibility is supported by $\beta$ beating measurements that have been performed with colliding beams. As of yet the deterministic correction of phase errors to reduce such an effect have not been tried.

\subsubsection{Beams crossing at an angle}

If the beams are crossing with a vertical or horizontal angle instead of using the beam sizes as in equation 65 one may use an effective height and a effective width given by $h_{\mathrm{eff}}=\sqrt{h^{2}+\sigma_{1}^{2} / \delta^{2}}$ and $w_{\text {eff }}=\sqrt{w^{2}+\sigma_{1}^{2} / \delta^{2}}$ where $\delta$ is one-half the angle between the beams and $\sigma_{1}$ is the bunch length [8]. In this case the luminosity due to imperfectly colliding Gaussian beams is given by:

$$
\mathcal{L}=\frac{f_{\text {rev }}}{4} \frac{N_{\mathrm{b}+} N_{\mathrm{b}-}}{A_{\text {int }}} \quad \text { where } \quad A_{\text {int }}=\frac{\pi}{4} N_{b} w_{\text {eff }} h_{\text {eff }} .
$$

One can see that the luminosity varies inversely proportionally to the effective beam height [8]. In LEP a vertical crossing angle of $100 \mu \mathrm{m}$, for example, will give a $5.5 \%$ loss in luminosity. Analysis at $45 \mathrm{GeV}$ in which a fit was made to difference orbit measurements through the insertions revealed small crossing angles. Similar analysis at $98 \mathrm{GeV}$ again showed crossing angles of less than $50 \mu \mathrm{m}$.

\section{Energy calibration}

The clean and well defined initial state in the centre-of-mass of the colliding beams make LEP an ideal place to measure the mass $m_{\mathrm{Z}}$ and the decay width $\Gamma_{\mathrm{Z}}$ of the $\mathrm{Z}$ boson as well as the mass $m_{\mathrm{W}}$ of the $\mathrm{W}$ boson. The determination of these electro-weak parameters requires however a precise knowledge of the centre-of-mass energy at the collision points. At a position $s$ along the ring the local beam momentum $p_{\mathrm{b}}(s)$, bending field $B(s)$ and radius of curvature $\rho(s)$ are related by

$$
\frac{1}{\rho(s)}=e \frac{B(s)}{p_{\mathrm{b}}(s)} .
$$

The momentum $p_{\mathrm{b}}(s)$ may differ locally from its average value $p_{\mathrm{b}}$ due to energy losses by synchrotron radiation and energy gains by the RF system. Closure of the orbit implies that the total bending angle $\theta$ integrated along the beam path must be exactly equal to $2 \pi$ :

$$
\oint d \theta=\oint \frac{d s}{\rho(s)}=e \oint \frac{B(s)}{p_{\mathrm{b}}(s)}=2 \pi .
$$

To a very good approximation the mean beam energy for ultra-relativistic particles is defined by the integrated bending field :

$$
E_{\mathrm{b}}=c p_{\mathrm{b}}=\frac{e c}{2 \pi} \oint B(s) d s .
$$


By design more than $99.9 \%$ of the integrated magnetic field of LEP is produced by the dipole magnets. Since keeping track of the magnetic fields becomes exceedingly difficult when relative accuracies of $10^{-5}$ are requested, the average beam energy is measured directly at LEP using the technique of resonant depolarization. An additional complexity arises for the energy in the centre-of-mass system which should ideally be exactly twice the beam energy. In practice the local energies of the two beams may differ at the collision points. Such shifts cannot be measured directly but must be evaluated using adequate models.

\subsection{Transverse polarization}

The build-up of transverse polarization in $\mathrm{e}^{+} \mathrm{e}^{-}$storage rings was first described by Sokolov and Ternov [53]. The emission of synchrotron radiation has a very small spin-flip probability, but a large asymmetry in favour of orienting the magnetic moment along the direction of the guiding magnetic field. In an ideal storage ring the maximum transverse polarization of $92.4 \%$ is building up with a rise time $\tau_{\mathrm{P}}$ :

$$
\tau_{\mathrm{P}}=\frac{8}{5 \sqrt{3}} \frac{m_{\mathrm{e}}^{6} c^{10} \rho^{3}}{\hbar r_{\mathrm{e}} E_{\mathrm{b}}^{5}} .
$$

At LEP the rise-time is 310 minutes for a beam energy of $46 \mathrm{GeV}$.

The motion of the spin vector $\vec{S}$ of a relativistic electron in electro-magnetic fields $\vec{E}$ and $\vec{B}$ is described by the Thomas-BMT equation [54] :

$$
\begin{aligned}
\frac{\mathrm{d} \vec{S}}{\mathrm{~d} t} & =\vec{\Omega}_{\mathrm{BMT}} \times \vec{S} \\
\vec{\Omega}_{\mathrm{BMT}} & =-\frac{e}{\gamma m_{\mathrm{e}}}\left[(1+a \gamma) \vec{B}_{\perp}+(1+a) \vec{B}_{\|}-\left(a \gamma+\frac{\gamma}{1+\gamma}\right) \vec{\beta} \times \frac{\vec{E}}{c}\right]
\end{aligned}
$$

where $\vec{B}_{\perp}$ and $\vec{B}_{\|}$are the components of the magnetic field which are transverse and parallel with respect to the particle's velocity $\vec{\beta} c . \vec{E}$ is the electric field and $a$ is the magnetic moment anomaly of the electron. In a storage ring the spin vector of a particle precesses $a \gamma$ times for one revolution, where the term $a \gamma$ is called the spin tune. Its average value over all particles $\nu$ is directly proportional to the average beam energy $E_{\mathrm{b}}$

$$
\nu=a \gamma=\frac{a E_{\mathrm{b}}}{m_{\mathrm{e}} c^{2}}=\frac{E_{\mathrm{b}}(\mathrm{MeV})}{440.6486(1)(\mathrm{MeV})} .
$$

The maximum polarization level is reduced in a real accelerator by resonances. The orbit and the tunes must be carefully set up and spin resonances $\nu=k Q_{\mathrm{x}}+l Q_{\mathrm{y}}+m Q_{\mathrm{s}}+n$, where $k, l, m, n$ are integers, must be avoided. The strong depolarizing effect of the beam-beam force for large beam-beam tune shifts prevents the buildup of transverse polarization at LEP during normal physics runs. Optimum polarization levels are achieved for beam energies corresponding to a spin tune close to a half-integer $\nu=n+1 / 2$ as shown in Figure 26. The record polarization level of $57 \%$ was observed at LEP for a beam energy of $44.72 \mathrm{GeV}[55,56]$. At higher energies the maximum polarization level is reduced by the large energy spread and the strong synchrotron spin resonances $\nu=n+k Q_{\mathrm{s}}$. So far polarization levels larger than $5 \%$ were only observed up to $60.6 \mathrm{GeV}$ [57]. The highest polarization measured at LEP as a function of the beam energy is shown in Figure 27.

The vertical polarization is measured using Compton scattering of a circularly polarized laser beam [58]. The $532 \mathrm{~nm}$ wavelength laser pulses of a frequency doubled Nd-Yag laser operated 


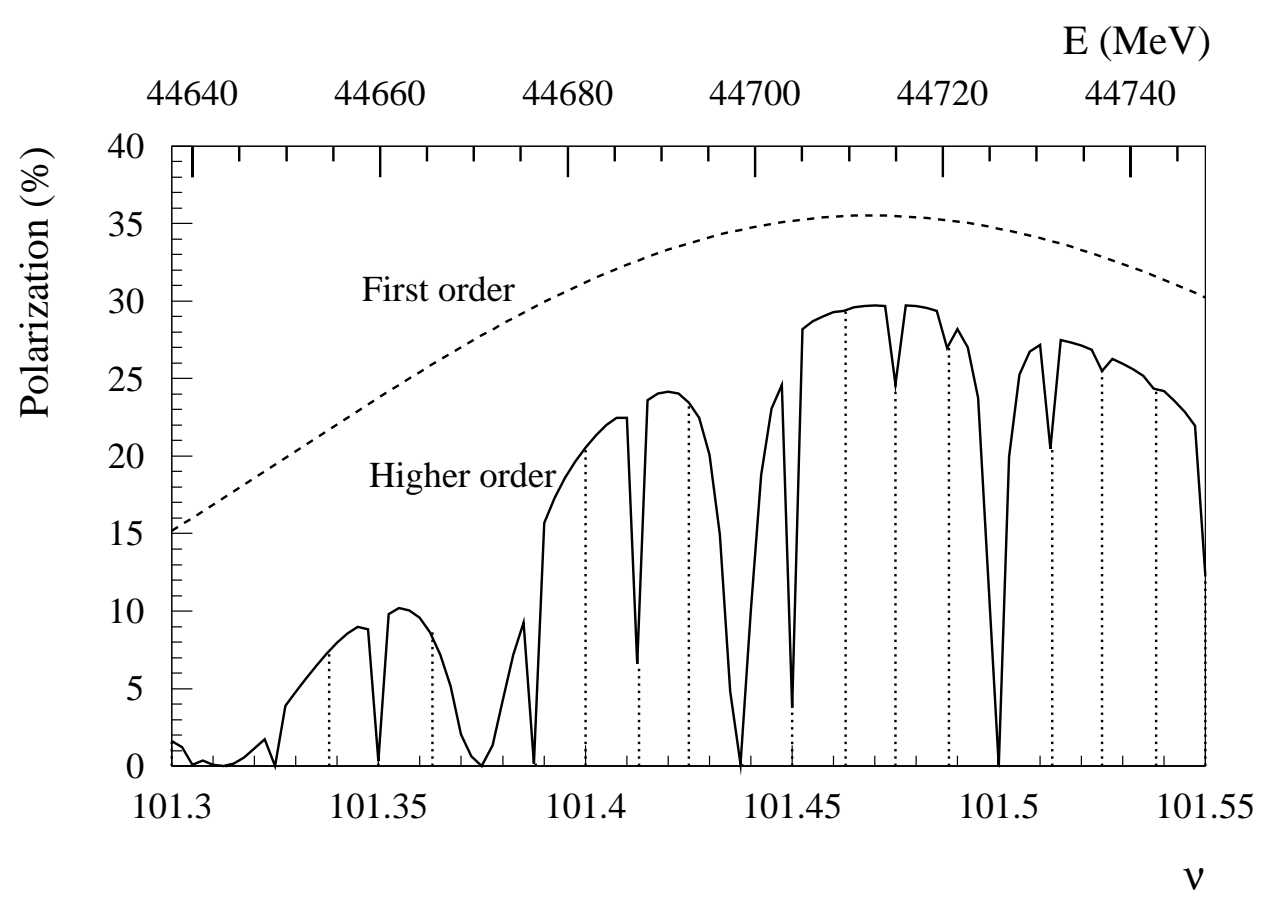

Figure 26: Transverse polarization level expected from a first order (linear) and a higher order calculations as a function of the spin tune. The polarization is highest when the spin tune is close to a half-integer value.

close to $100 \mathrm{~Hz}$ are scattered under a very small angle $(3 \mathrm{mrad})$ on the particle bunches. The distribution of the back-scattered photons is a function of the laser and the beam polarization. The vertical profile of the scattered photon pulse is detected using a sampling calorimeter made of Silicon strip detectors and tungsten plates located about $300 \mathrm{~m}$ downstream of the collision point. The centre-of-gravity shift obtained when the circular polarization of the laser light is flipped is directly proportional to the polarization. A $10 \%$ beam polarization yields a shift of the vertical distribution of approximatively $50 \mu \mathrm{m}$ for the LEP setup.

\subsection{Energy calibration by resonant depolarization}

For transversely polarized beams the relation between spin tune and energy, see Equation 72, allows accurate measurements of the average beam energy. The spin precession frequency is measured with an RF-magnet whose radial field strength is used to rotate the spins. When the deflections from the RF-magnet are in phase with the spin precession, the spin rotations add up coherently from turn to turn. Ideally the polarization can be flipped, but in general the beam is just depolarized because the horizontal component of the polarization has a very short decay time. The RF-magnet field oscillating at a frequency $f_{\text {dep }}$ is in resonance with the spin precession if

$$
f_{\text {dep }}=(k \pm[\nu]) f_{\text {rev }}
$$

where $k$ is an integer. $[\nu]$ denotes the non-integer (fractional) part of the spin tune. The integer part of $\nu$ must be deduced from the calibration of the bending magnets.

At LEP the frequency of the RF-magnet field is slowly varied with time over a given range. The width of the frequency scan $\Delta f_{d e p}$ is usually set to $22 \mathrm{~Hz}$ which corresponds to $\Delta \nu=0.002$ or $\Delta E=0.88 \mathrm{MeV}$ [59]. The frequency scans are repeated until a depolarization is observed 


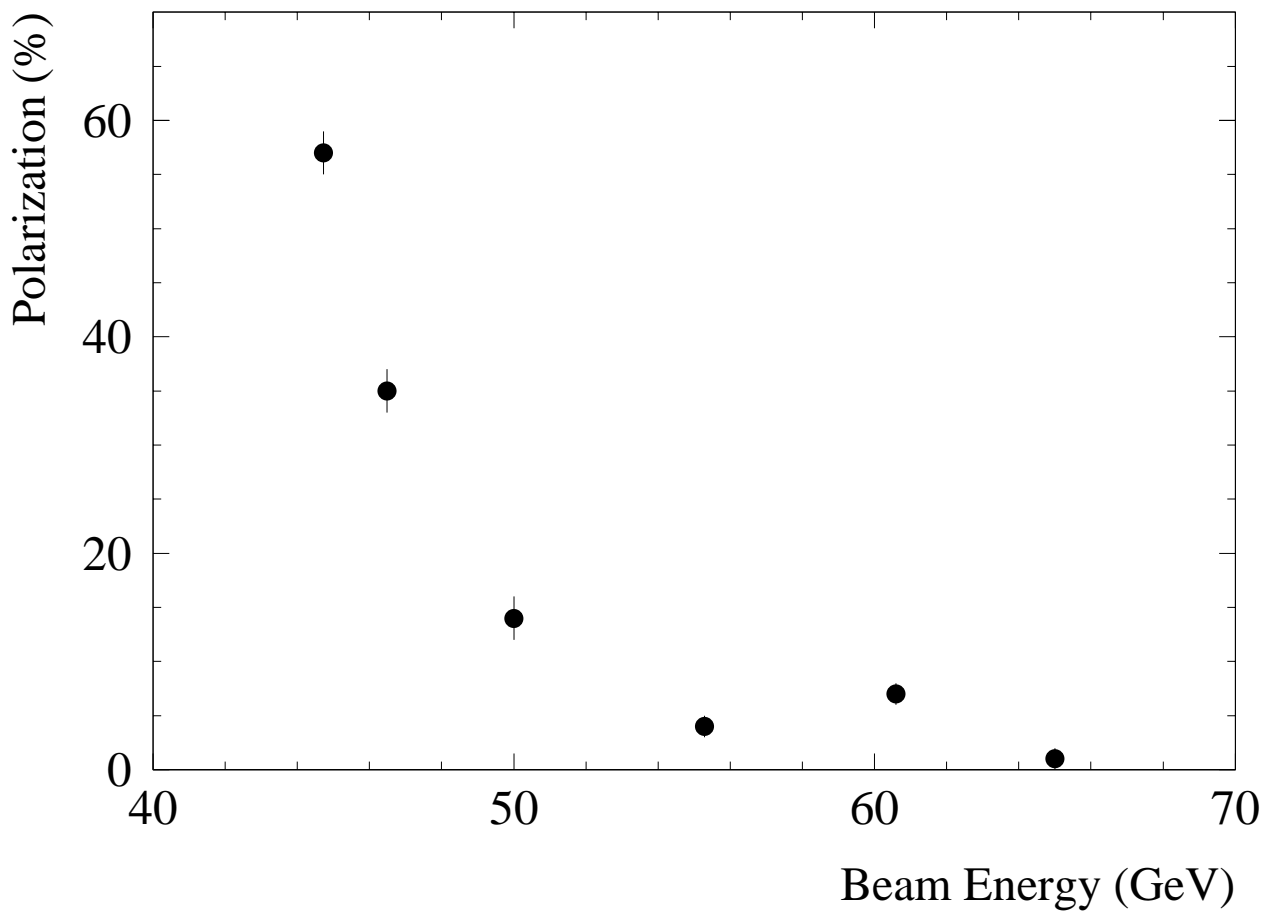

Figure 27: Highest transverse polarization level observed at LEP as a function of beam energy. The strong decrease with beam energy is clearly visible.

in a given frequency bin. This method is referred to as energy calibration by resonant depolarization and has been used extensively for accurate beam energy calibrations and measurements of particle masses [60]. The polarization vector is an average over all spin vectors, hence the measured beam energy is independent of betatron and synchrotron oscillations of the individual particles and is not limited in accuracy by the LEP beam energy spread. Local energy variations like the energy sawtooth do not bias the measured beam energy. At LEP a minimum polarization level of about $5 \%$ is required to observe clean depolarizations and determine the beam energy.

\subsection{Z boson resonance scans}

For precise measurements of $\Gamma_{\mathrm{Z}}$ and $m_{\mathrm{Z}}$ the beam energy of LEP was scanned around the $\mathrm{Z}$ boson resonance [61, 62]. The two main scans were performed in the 1993 and 1995 LEP runs and aimed at accuracies on the centre-of-mass energy of $\approx 1-2 \times 10^{-5}$. The ability to polarize the beam constrained the choice of beam energy, which was scanned over 3 points corresponding roughly to the peak of the $\mathrm{Z}$ resonance $(\nu \simeq 103.5)$ and to two points located $0.9 \mathrm{GeV}$ above and below the resonance peak $(\nu \simeq 101.5$ and 105.5) as shown in Figure 28. Since high luminosity operation is not compatible with beam polarization, the energy calibrations were made regularly at the end of the physics fills with non-colliding beams and minimal changes to the machine conditions. Dedicated experiments were also performed to investigate various aspects of the energy modelling. The large amount of data accumulated between 1991 and 1995 unveiled many subtle effects that were influencing the LEP beam energy at the $10^{-4}$ level, more than one order of magnitude larger than the desired accuracy. The most important effects will be described below. 


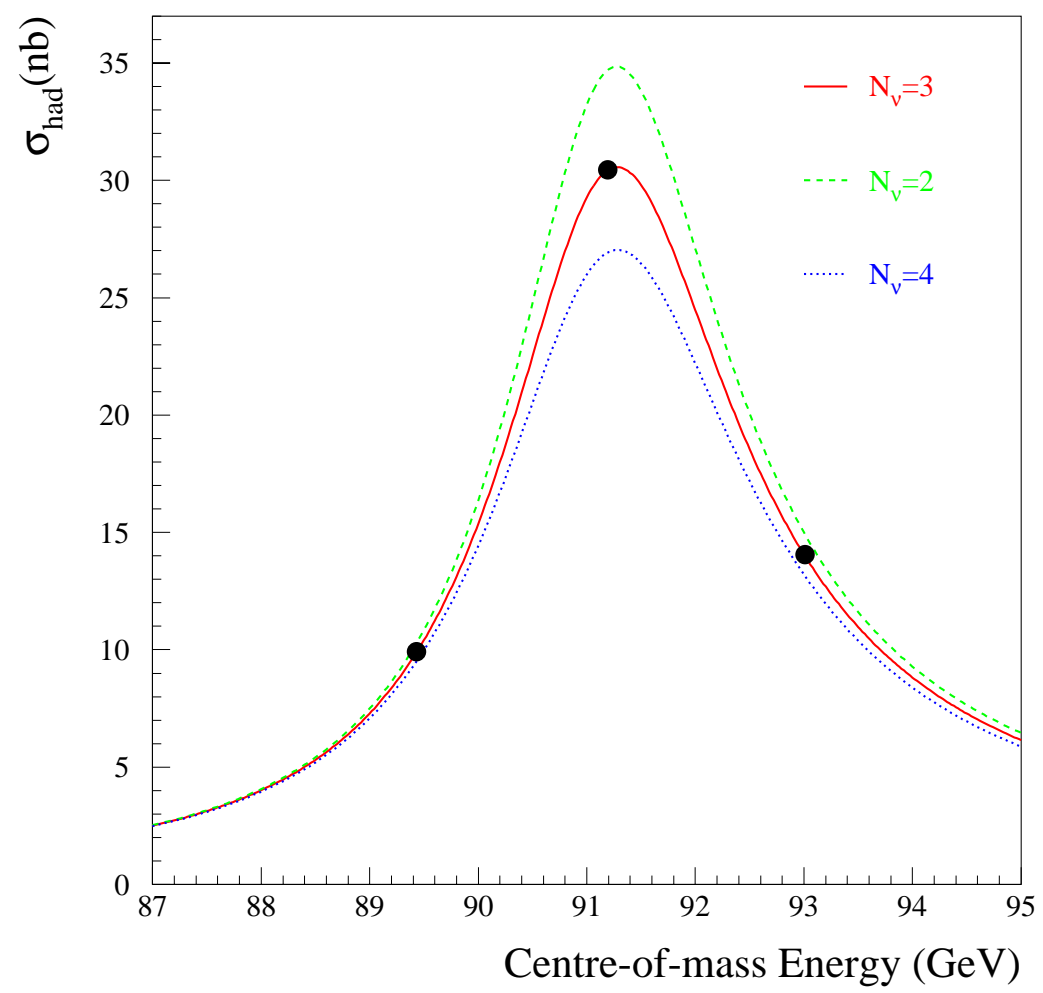

Figure 28: Cross section for the production of events with hadrons at LEP as a function of the centre-of-mass energy around the $\mathrm{Z}$ boson resonance. The maximum of the curve corresponds approximatively to the mass of the $\mathrm{Z}$ boson. The three points $(\bullet)$ correspond to energies compatible with beam polarization which have been accurately calibrated by resonant depolarization in 1993 and 1995. The curves indicate the expected cross-sections for 2, 3 and 4 families of light neutrinos.

\subsubsection{Magnet ic fiel d stabil ity}

In 1995 a perturbation of the dipole field was observed for the first time when Nuclear Magnetic Resonance (NMR) probes were installed in 2 tunnel dipoles to monitor their fields. Large short term fluctuations and long-term field increases corresponding to $\Delta B / B=\Delta E_{\mathrm{b}} / E_{\mathrm{b}} \simeq 2 \times 10^{-4}$ were observed by the 2 tunnel probes (Figure 29) over 12 hours. In contrast probes installed in a reference magnet located in a surface building and connected in series with the LEP ring dipoles registered fluctuations smaller by about one order of magnitude. The short term noise consisting of low frequency $(<1 \mathrm{~Hz})$ fluctuations shows a repetitive daily pattern with a noisefree period between midnight and about 4:30 am. The long term upward drift is driven by the short term noise.

The perturbation could be attributed to leakage currents flowing on the LEP vacuum chamber [63]. The currents are generated by trains circulating on a French railway line linking the cities of Geneva and Lyon which is operated at $1.5 \mathrm{kV}$ DC. In contrast Swiss railway lines are operated at $15 \mathrm{kV}$ AC. For a given engine power the DC currents are 10 times higher and the diffusion (leakage) of DC current into the ground is large, with up to $25 \%$ of the current not returning to the generator over the railway tracks. The exact amount of current seeping into the ground depends on the electrical insulation of the railway tracks. Metal conductors like pipes, wells and rivers can carry the parasitic currents. For LEP the currents enter the tunnel around IP6 and exit the tunnel over the transfer lines to the SPS near IP1, see Figure 1. The currents flow clockwise from IP6 via IP8 to IP1, anti-clockwise via IP4. The currents themselves 


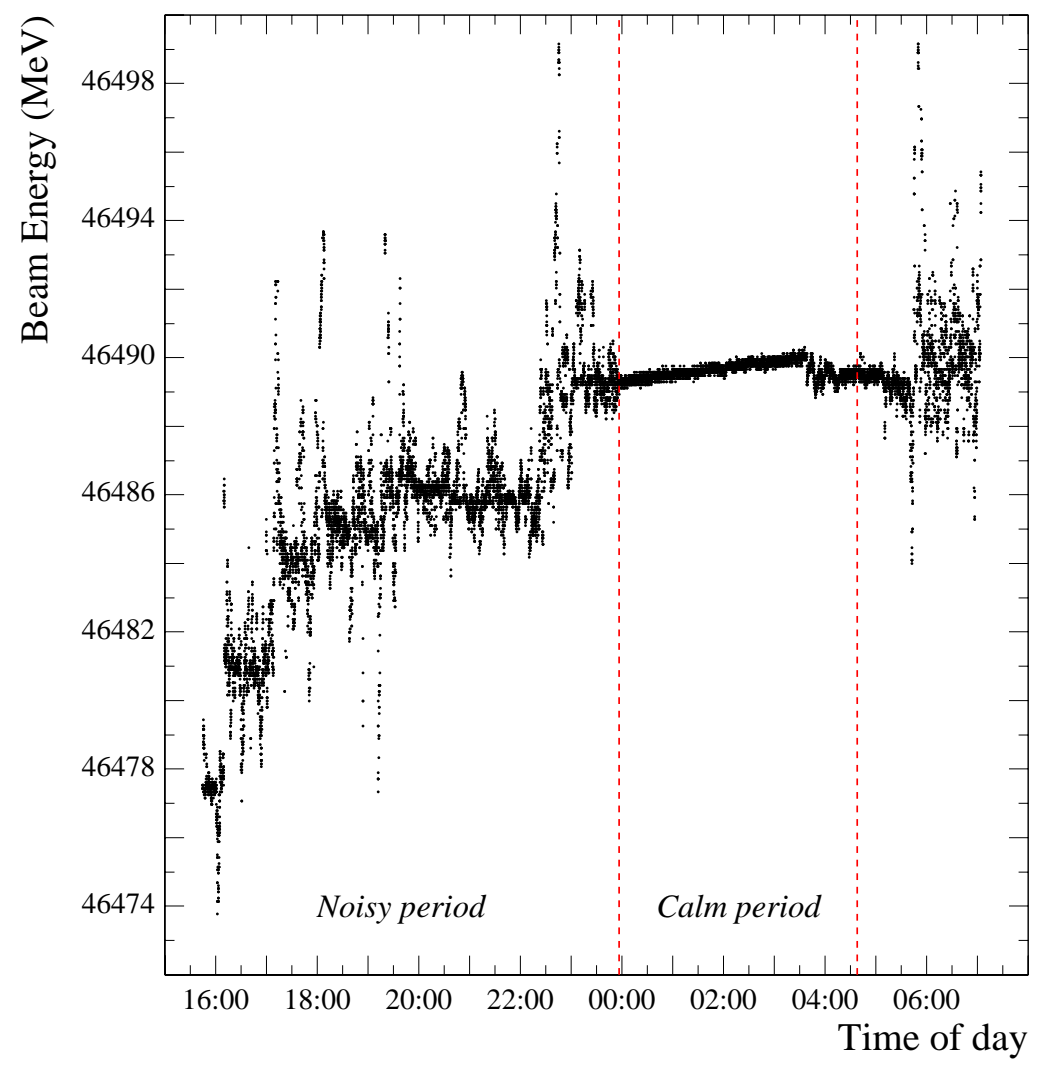

Figure 29: Magnetic field measured in a LEP dipole by an NMR probe over 10 hours. For convenience the magnetic field has been converted to an equivalent beam energy in MeV. Large short term fluctuations and a slow rise in field are clearly visible. Between midnight and 4:30 am the field is stable while the fluctuations disappear.

do not induce energy changes of the beam because the net current averages to zero over the circumference. The dipole field however does not drop back to its initial value after a current spike but remains at a slightly higher value due to the magnet hysteresis curve. A succession of currents spikes induces a slow increase of the field and the beam energy over time. The field in the magnets finally saturates due to the finite amplitude of the spikes.

\subsubsection{Circumf er ence variations and tides}

In LEP the particles are ultra-relativistic and their orbit length is determined by the frequency of the RF system. Following a circumference change $\Delta C$, the beam is forced to adapt its radial position in the magnets since it cannot change its speed. The additional field sampled by the beam in the quadrupoles leads to a change in energy $\Delta E_{\mathrm{b}}$

$$
\frac{\Delta E_{\mathrm{b}}}{E_{\mathrm{b}}}=-\frac{1}{\alpha_{\mathrm{c}}} \frac{\Delta C}{C}
$$

where the momentum compaction factor $\alpha_{\mathrm{c}}$ varies between $1.4 \times 10^{-4}$ and $3.9 \times 10^{-4}$. Because of its large size and the small value of $\alpha_{\mathrm{c}}$ LEP is sensitive to geological movements generated by tides and hydrological strains.

Tides are caused by the $1 / r^{2}$ dependence of the gravitational attraction of the sun and the moon. The presence of a celestial body leads to a quadrupolar deformation of the Earth 
surface. The radius change $\Delta R$ is

$$
\Delta R \sim \frac{M}{2 a^{3}}\left(3 \cos ^{2} \theta-1\right)
$$

where $\theta$ is the angle between the vertical and the celestial body and $a$ is the distance to the body of mass $M$. Sun tides are about $50 \%$ weaker than Moon tides due to the cubic dependence on the distance. The horizontal strain associated to the tidal forces induces peak-to-peak circumference changes of up to $1 \mathrm{~mm}$. Figure 30 shows the result of a controlled experiment during a period of full Moon. The beam energy variation follows the expected tidal distortions. The large knowledge accumulated in the past 50 years on Earth tides allowed to model the tidal circumference and energy changes with very good accuracy [64].

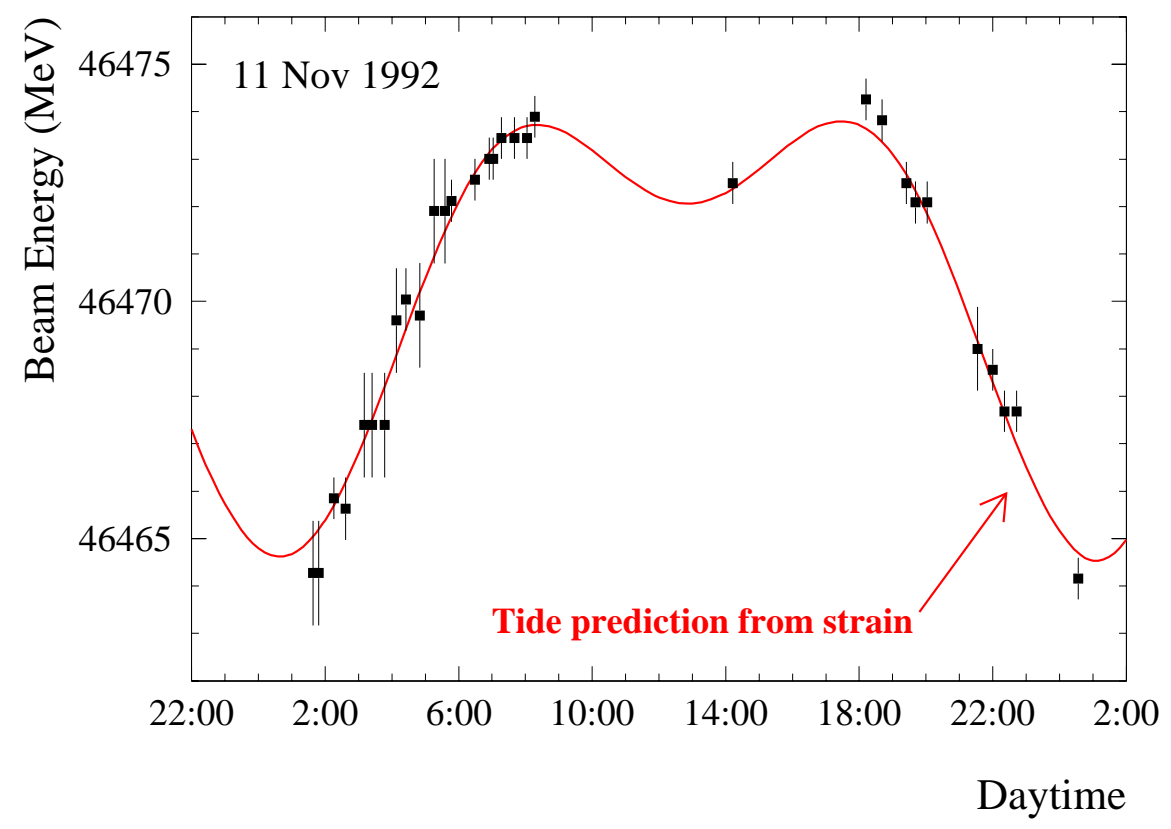

Figure 30: Energy variation of the LEP beams during a full Moon day. The curve is the energy change predicted from the horizontal strain induced by the Earth tides.

Besides the periodic tidal movements LEP is also subject to much slower long term circumference changes [65]. Such movements can be monitored by observing the radial movement of the beam relative to the quadrupoles in the beam position monitors. During a typical run lasting from May to November the LEP ring experiences circumference changes of up to $2 \mathrm{~mm}$ as shown in Figure 31. The general trend is reproducible from one year to the next. The circumference usually increases during the Summer months, some of the changes being clearly correlated to rainfall and to fluctuations in the underground water table height. Predictions of $\Delta C$ are not possible with good accuracy because the geological problem is complex and too many variables are unknown. The monitoring of the circumference is therefore relying on the beam position measurements alone. The monitoring of those seasonal variations of the circumference turned out to be very important to understand the evolution LEP beam energy since the associated energy variations reach $\Delta E_{\mathrm{b}} / E_{\mathrm{b}} \approx 5 \times 10^{-4}$. 


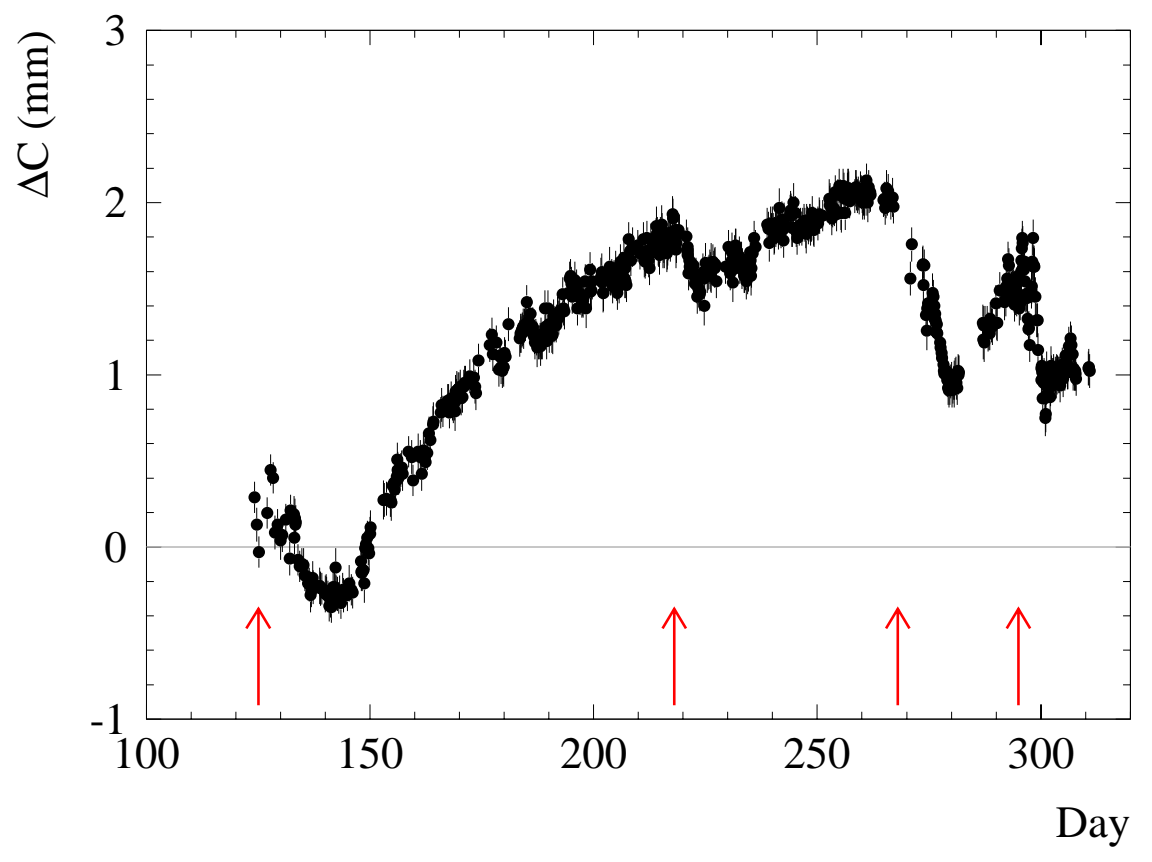

Figure 31: Evolution of the LEP circumference (corrected for tidal changes) as a function of the day in 1999. A drift of over to $2 \mathrm{~mm}$ is observed during the LEP run. In the summer months the circumference increases gradually. Following periods of heavy rainfall, indicated by the arrows, the circumference shrinks for some time before expanding again.

\subsubsection{Centre-of-mass energies}

At the collision points of the beams the quantity relevant for all physical processes is the centre-of-mass energy which is obtained from the average beam energy determined by resonant depolarization after correction for two local effects.

A first cause for centre-of-mass energy shifts is due to longitudinal cavity misalignments, RF phase errors between cavities as well as the actual voltage distribution. Typical corrections and fluctuations are smaller than $10 \mathrm{MeV}$, although a large systematic centre-of-mass energy shift of $16 \mathrm{MeV}$ was introduced accidently at the 2 IPs where the LEP1 Copper RF cavities were installed. The origin of the effect lies in a systematic longitudinal alignment error of these cavities. A complete model of the RF system is used to predict the correction to the centreof-mass energy as well as the following observable quantities : the synchrotron tunes of the 2 beams, the longitudinal positions of the collision points and the horizontal orbit differences around the IPs induced by the local energy differences. Comparisons of predicted and measured observables are used to tune the model and evaluate systematic errors on the centre-of-mass energy.

Vertical dispersion is at the origin of a second correction to the centre-of-mass energy. The electrostatic separators used to avoid parasitic encounters of the beams generate vertical dispersion of opposite sign for the $\mathrm{e}^{+}$and $\mathrm{e}^{-}$beams. If the beams collide with a vertical offset, the correlation between transverse position and energy leads to centre-of-mass energy shifts $\Delta E_{\mathrm{CM}}[47,62,66]$ :

$$
\Delta E_{\mathrm{CM}}=-\frac{1}{2} \frac{\delta y^{*}}{\sigma_{\mathrm{y}}^{2}} \frac{\sigma_{\mathrm{e}}^{2}}{E_{\mathrm{b}}} \Delta D_{\mathrm{y}}^{*}
$$

where $\delta y^{*}$ is the collision offset between the two beams and $\Delta D_{\mathrm{y}}^{*}$ is the dispersion difference at 
the IP. By the same effect the centre-of-mass energy spread is also reduced.

This 'monochromator effect' is particularly important when LEP is operated with bunch trains in the vertical plane where the beam sizes are small and significant differences in dispersion are expected. Because the horizontal beam size at the IP is rather large $(>200 \mu \mathrm{m})$, the $\mathrm{CM}$ shifts are very small in that plane. To keep centre-of-mass energy shifts due to opposite sign dispersion under control, the collision offsets had to be carefully minimized with regular luminosity scans.

\subsubsection{Summary of LEP 1 energy calibration}

A model of the LEP beam and centre-of-mass energies was developed from the large amount of energy calibration data accumulated over the years. The models were used to extrapolate beam energies in time between energy calibrations. Best possible estimates of the energies over 15 minute intervals were given to the LEP experiments for data analysis. The final result of this work was a systematic error due to the energy calibration of the LEP beams of only $1.7 \mathrm{MeV}$ on $m_{\mathrm{Z}}$ and of $1.3 \mathrm{MeV}$ on $\Gamma_{\mathrm{Z}}[62]$. The originally anticipated accuracy on $m_{\mathrm{Z}}$ was $\simeq 20 \mathrm{MeV}$.

\subsection{Energy calibration at LEP2}

At LEP2 a direct energy calibration by resonant depolarization is not possible because transverse polarization does not build up at such high energies, see Figure 27. The beam energy has to be extrapolated from measurements performed at lower energies between 41 and $60 \mathrm{GeV}$.

Since the accuracy on the energies depends mainly on the understanding of the dipole field, 16 NMR probes were installed in LEP tunnel dipoles. The NMR probes are cross-calibrated with resonant depolarization between 41 and $60 \mathrm{GeV}$ and used to predict the energy in physics conditions. So far this technique yielded systematic errors of 15 to $20 \mathrm{MeV}$ per beam, slightly above the desired accuracy of $10 \mathrm{MeV}$. The main uncertainty stems from the understanding of the dipole field interpolation which is hampered by the long lever arm between the highest energy calibration point at $60 \mathrm{GeV}$ and the operating energies of 80 to $100 \mathrm{GeV}$ [67].

A calibration of the beams based on the relation between the synchrotron tune $Q_{\mathrm{s}}$ and the total accelerating voltage is presently being developed. This method may reach accuracies of $20 \mathrm{MeV}$ at beam energies of $90 \mathrm{GeV}$ [68].

\subsubsection{The LEP spectromet er}

To avoid the problem of field interpolation a spectrometer was installed in the LEP ring to reduce the beam energy uncertainty and provide an independent energy measurement. Two normal LEP dipoles were replaced by a dedicated dipole whose integrated field was determined to a relative accuracy of $\approx 3 \times 10^{-5}$. The dipole is instrumented with 4 NMR probes to monitor absolute field variations. Three high resolution beam position monitors are installed on either side of the dipole to measure the beam angles. Because the beam positions must be measured with relative accuracies of about 1 micrometer at various beam energies, the monitors must be cooled and stabilised in temperature. At this level of accuracy the position mesurement becomes also sensitive to the beam dimensions.

The spectrometer beam position monitors are first calibrated between 40 and $60 \mathrm{GeV}$ by resonant depolarization. Following a successful calibration the beams are ramped to higher energies where a differential measurement of the beam angle is performed. Preliminary results indicate that accuracies below $15 \mathrm{MeV}$ may be achieved. 


\section{Summary of LEP performance}

The primary aims of LEP are to exploit fully the available energy range while delivering as much luminosity to the experiments as possible. At a given energy the luminosity may be increased by : increasing the bunch current, increasing the number of bunches, and minimizing the horizontal and vertical beam sizes while necessarily respecting the limits imposed by beambeam, the equipment, instabilities etc. In this respect the LEP years naturally divide into two regimes : $45 \mathrm{GeV}$ running around the $\mathrm{Z}$ boson resonance and high energy running above the threshold for $\mathrm{W}$ pair production.

The performance at $45 \mathrm{GeV}$ was very much constrained by the beam-beam effect which limited the bunch current that could be collided. Optimization of the transverse beam sizes was also limited by beam-beam driven effects such as flip-flop and operationally life was a struggle as the beam-beam limit was continually probed. The main break-through in performance at this energy was an increase in the number of bunches. First with the Pretzel scheme ( 8 bunches per beam) which was commissioned in 1992, and then the bunch train scheme (up to 12 bunches per beam) which was used in 1995. Both schemes, although ambitious, were used successfully and, indeed bunch trains continue to be used at $45 \mathrm{GeV}$ for detector calibrations. Both the Pretzel and bunch trains schemes reduced the bunch current that was collided and sometimes also the resultant beam-beam tune-shift, however the increase in the number of bunches provided a net gain. The peak luminosities were well in excess of the design figure of $17 \times 10^{30} \mathrm{~cm}^{-2} \mathrm{~s}^{-1}$.

With an increase in energy the effect of the beam-beam force falls and higher bunch currents can be collided. At the same time stronger damping raises the beam-beam limit itself. This release from the tight constraints of beam-beam are clearly reflected in the increase in peak luminosity and peak beam-beam tune shifts as LEP moved to higher energies. Firstly because higher bunch currents are allowed, the use of a fewer number of bunches was possible - a maximum of 4 bunches per beam were used - and secondly thorough optimization of vertical beam size was now possible. Both coupling and residual vertical dispersion were targeted and the maximum instantaneous luminosity and beam-beam tune shifts as a result ended by exceeding all expectations.

Table 6: Overview of LEP (instantaneous) peak performance 1989-1999. $\int \mathcal{L} d t$ is the luminosity integrated per experiment over each year. The design luminosity at $45 \mathrm{GeV}$ is $17 \times 10^{30} \mathrm{~cm}^{-2} \mathrm{~s}^{-1}$.

\begin{tabular}{ccccccc}
\hline Year & $\begin{array}{c}\int \mathcal{L} d t \\
\left(\mathrm{pb}^{-1}\right)\end{array}$ & $\begin{array}{c}E_{\mathrm{b}} \\
\left(\mathrm{GeV} / \mathrm{c}^{2}\right)\end{array}$ & $k_{\mathrm{b}}$ & $\begin{array}{c}2 k_{\mathrm{b}} I_{\mathrm{b}} \\
(\mathrm{mA})\end{array}$ & $\begin{array}{c}\mathcal{L} \\
\left(10^{30} \mathrm{~cm}^{-2} \mathrm{~s}^{-1}\right)\end{array}$ & $\xi_{\mathrm{y}}$ \\
\hline 1989 & 1.74 & 45.6 & 4 & 2.6 & 4.3 & 0.017 \\
1990 & 8.6 & 45.6 & 4 & 3.6 & 7 & 0.020 \\
1991 & 18.9 & 45.6 & 4 & 3.7 & 10 & 0.27 \\
1992 & 28.6 & 45.6 & $4 / 8$ & 5.0 & 11.5 & 0.027 \\
1993 & 40.0 & 45.6 & 8 & 5.5 & 19 & 0.040 \\
1994 & 64.5 & 45.6 & 8 & 5.5 & 23.1 & 0.047 \\
1995 & 46.1 & 45.6 & $8 / 12$ & 8.4 & 34.1 & 0.030 \\
1996 & 24.7 & 80.5 to 86 & 4 & 4.2 & 35.6 & 0.040 \\
1997 & 73.4 & 90 to 92 & 4 & 5.2 & 47.0 & 0.055 \\
1998 & 199.7 & 94.5 & 4 & 6.1 & 100 & 0.075 \\
1999 & 253 & 98 to 101 & 4 & 6.2 & 100 & 0.083 \\
\hline
\end{tabular}

As mentioned above the bunch currents which could be collided at $45 \mathrm{GeV}$ were strongly limited by beam-beam. It was initially hoped that at higher energies bunch currents of up to $1 \mathrm{~mA}$ might be used, and an extensive program of machine experiments succeeded in accumulating these bunch currents at injection. However in the end more practical limitations, 
Table 7: Bunch schemes and optics used at LEP.

\begin{tabular}{cccc}
\hline Year & Optics & Comment & Bunch scheme \\
\hline 1989 & $60^{\circ} / 60^{\circ}$ & LEP commissioned & 4 on 4 \\
1990 & $60^{\circ} / 60^{\circ}$ & & 4 on 4 \\
1991 & $60^{\circ} / 60^{\circ}$ & $90^{\circ} / 90^{\circ}$ optics tested & 4 on 4 \\
1992 & $90^{\circ} / 90^{\circ}$ & Pretzel commissioned & 4 on $4 /$ Pretzel \\
1993 & $90^{\circ} / 60^{\circ}$ & & Pretzel \\
1994 & $90^{\circ} / 60^{\circ}$ & & Pretzel \\
1995 & $90^{\circ} / 60^{\circ}$ & tests at $65-68 \mathrm{GeV}$ & Bunch trains \\
1996 & $90^{\circ} / 60^{\circ}$ & $108^{\circ} / 90^{\circ}$ tested & 4 on 4 \\
1997 & $90^{\circ} / 60^{\circ}$ & $108^{\circ} / 60^{\circ}$ and $102^{\circ} / 90^{\circ}$ tested & 4 on 4 \\
1998 & $102^{\circ} / 90^{\circ}$ & & 4 on 4 \\
1999 & $102^{\circ} / 90^{\circ}$ & & 4 on 4 \\
\hline
\end{tabular}

such as RF system stability, restricted the bunch currents in physics to a maximum of around $0.780 \mathrm{~mA}$. As the RF system was pushed to higher accelerating gradients this figure fell even further.

Turn-around between physics fill, reproducibility, ramp efficiency and equipment reliability were all tackled over the years and improvements in these areas were important in improving the overall performance.

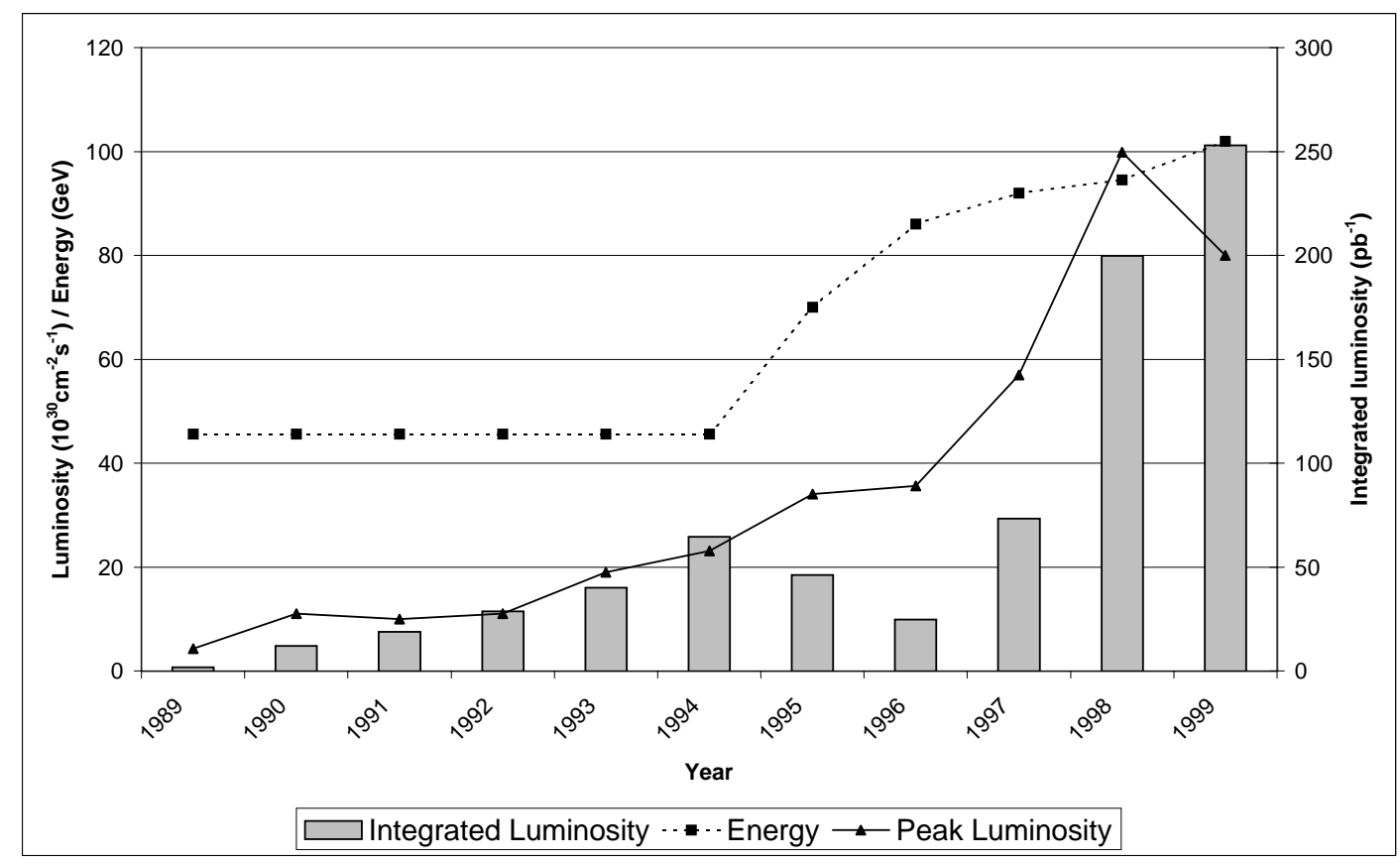

Figure 32: Maximum energy, maximum luminosity and integrated luminosity per experiment for each year between 1989 and 1999.

Tables 6 and 7 and Figures 32 and 33 summarise the key performance indicators year by year. The following salient points may be noted:

- the slow improvement in the first three years while running 4 bunches on 4 bunches at $45 \mathrm{GeV}$, 


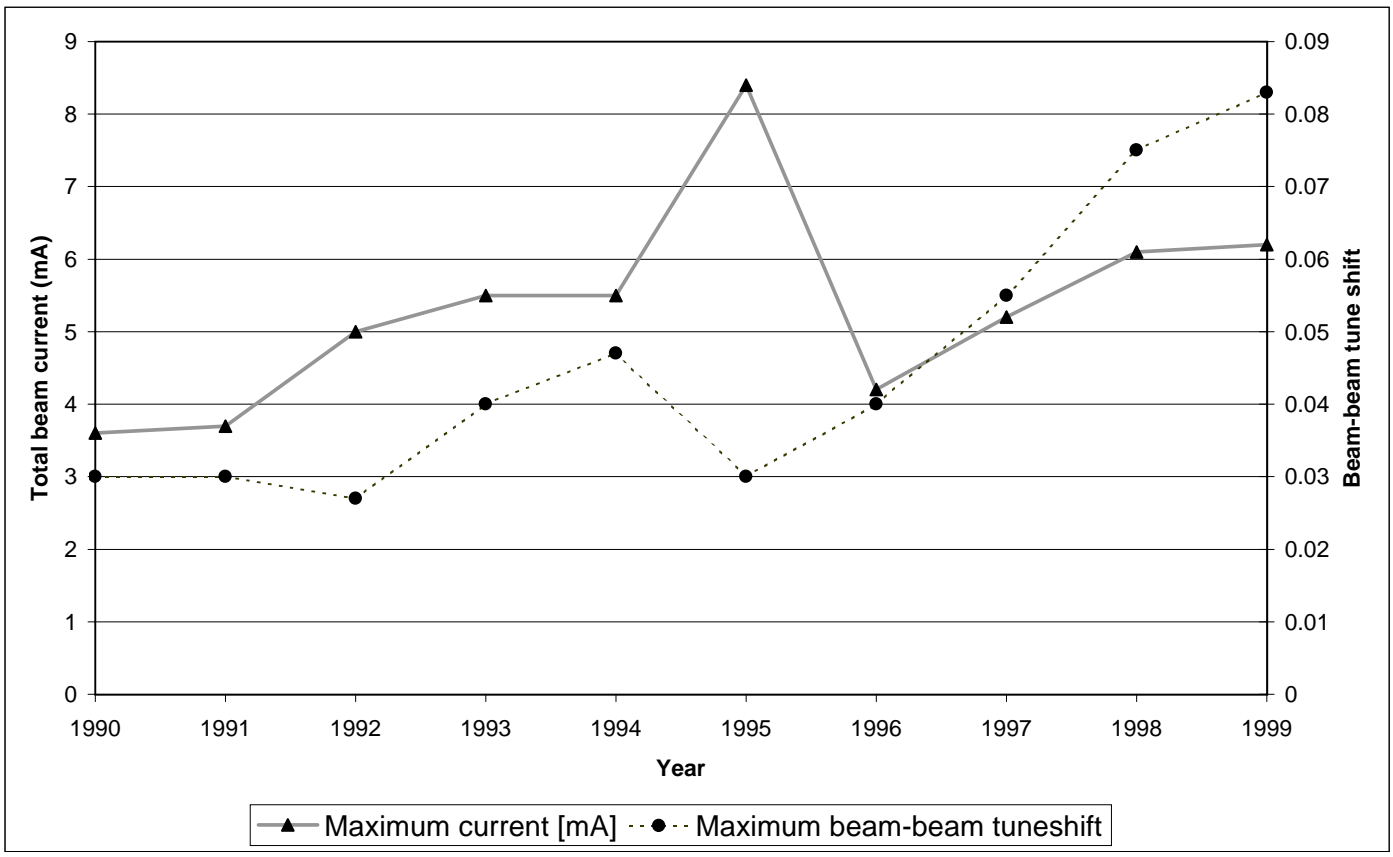

Figure 33: Evolution of maximum total current in collision and the maximum beam-beam tune shift between 1990 and 1999.

- the cost of introducing a new multi-bunch scheme which takes a full year to reach fruition,

- the sizable increase in beam-beam tune shift when higher energies un-shackled the constraints of beam-beam,

- the increase in delivered luminosity despite not increasing the beam current; partially due to the increased instantaneous performance and partially to increased operational efficiency.

- the evolution of the beam optics; the horizontal phase advance was progressively increased to adapt the horizontal beam emittance for best performances.

By the end of 1999 LEP had delivered to each experiment an integrated luminosity over over $200 \mathrm{pb}^{-1}$ around $45 \mathrm{GeV}$ and over $500 \mathrm{pb}^{-1}$ above $\mathrm{W}$ production threshold. This corresponds to over 4 million $\mathrm{Z}$ boson and over $7000 \mathrm{~W}$ boson pair events per experiment.

The beam energy at LEP2 reached $101 \mathrm{GeV}$ in 1999, with hopes to push the limit to $103 \mathrm{GeV}$ or more in the year 2000. The main aim of this effort is the search for the Higgs boson and for super-symmetric particles [69]. Current estimates, based on existing experimental data, predict a Higgs mass of $77_{-39}^{+69} \mathrm{GeV} / \mathrm{c}^{2}$ [1]. In 2000 LEP could become sensitive to Higgs masses as high as $114 \mathrm{GeV} / \mathrm{c}^{2}$.

The precision of the LEP beam energy was reduced by more than one order of magnitude with respect to original estimates with resonant depolarization. This achievement was obtained after over six years of "detective" work : subtle influences of Earth tides, geological movements and leakage currents from railway trains had to be unravelled. As a direct consequence, the mass of the $\mathrm{Z}$ boson is now one of the most accurately known parameters of the electroweak theory, and the precise determination of the $\mathrm{Z}$ boson decay width made a prediction of the top quark mass possible to within a few $\mathrm{GeV} / \mathrm{c}^{2}[1]$. The results from the LEP experiments have been used extensively to test the Standard Model of particle physics. 


\section{Appendix and symbols}

Table 8: List of frequently used symbols. Subscripts $(+)$ and $(-)$ are used to distinguish positron and electron beams.

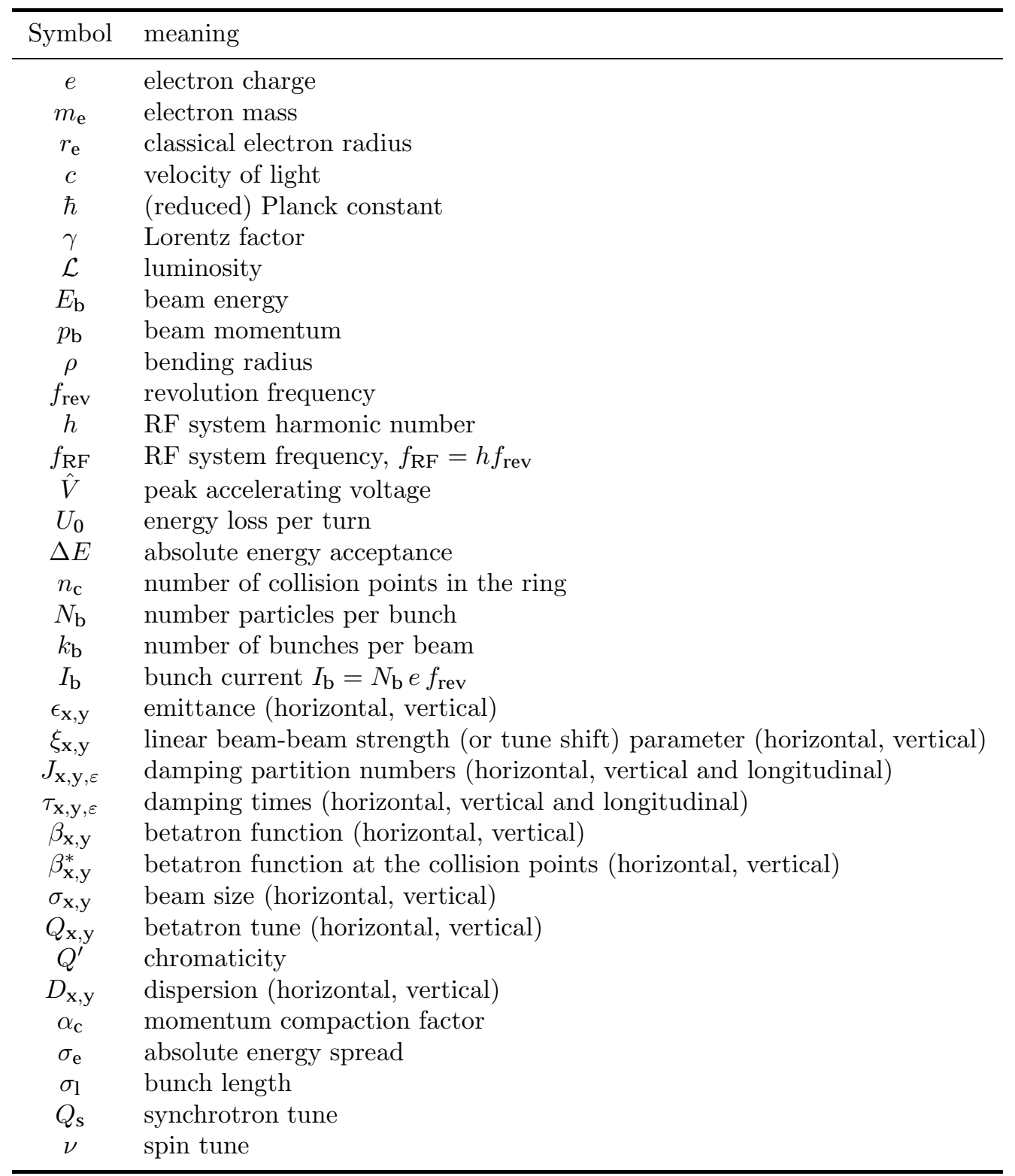




\section{References}

\section{References}

[1] The LEP Collaborations et al 2000 "A combination of preliminary Electroweak measurements and constraints on the Standard Model" CER N Report CERN-EP-2000-016

[2] Richter B 1976 Nucl. Instrum. Methods 13647

[3] The LEP study Group 1978 "Design study of a 15 to $100 \mathrm{GeV} \mathrm{e}^{+} \mathrm{e}^{-}$colliding beam machine (LEP)" CER N Report ISR-LEP/78-17

[4] The LEP study Group 1979 "Design study of a 22 to $130 \mathrm{GeV} \mathrm{e}^{+} \mathrm{e}^{-}$colliding beam machine (LEP)" CER N Report ISR-LEP/79-33

[5] LEP 1983 "The LEP injector chain" The LEP design report Vol. I CERN Report $\mathrm{LEP} / \mathrm{TH} / 83-29$

LEP 1984 "The LEP main ring" T he LEP design report Vol.II CER N Report LEP/84-01 LEP 1996 "LEP2" The LEP design report Vol.III CERN Report AC/96-01

[6] Brown P et al 1999 "Performance of the LEP200 superconducting RF system" CER N Report CERN-SL-99-075-LRF

[7] Arduini G et al. 1996 "LEP1 operation, 1989-1995" Proc. of the 5th European Particle Accelerator Conf. (Sitges) ed V. Suller and C. Petit-Jean-Genaz (Bristol : IOP) pp. 286288

[8] M. Sands 1970 "The Physics of Electron Storage Rings: An Introduction" SLAC-121

[9] Wiedemann H 1995 Particle Accelerator Physics I and II (Springer)

[10] Müller M 2000 "Precision Measurements of the LEP Beam Energy for the Determination of the W Mass" PhD thesis University of Mainz

[11] Arduini G et al. 1997 "Parasitic energy loss in the LEP superconducting cavities" Proc. of the 17th Particle Accelerator Conf. (Vancouver) ed M. Comyn et al. (New York: IEEE) pp 1858-1860

[12] Burkhardt H 1999 "Beam lifetime and beam tails in LEP" CER N Report CERN-SL-99061-AP

[13] Littauer R M 1985 "Multibunch operation of CESR" IEEE Trans. Nucl. Sci. 32 p 1610

[14] Jowett J M, Kalbreier W and Myers S 1990 "Preparations for high-luminosity LEP" Proc. of the 2nd European Particle Accelerator Conf. (Nice) ed P. Marin and P. Mandrillon (Ed. Frontieres) pp 403-405

[15] Goddard B et al. 1997 Part. Accel. 57237

[16] Verdier A 1995 "Chromaticity" CER N Accelerator School 5th advanced accelerator physics course Vol.I CER N Yellow Report 95-06

[17] Myers S Nucl. Instrum. and M ethods 211 (1983) 263-282

[18] von Holtey G et al 1998 Nucl. Instrum. and Methods A 403 205-246

[19] Verdier A 1994 "Reduction of $\beta^{*}$ " Proc. of the 4th W orkshop on LEP Performance (Chamonix) ed J Poole CER N Report CERN-SL/94-06 (DI) p 289

[20] Helm R H, Lee M J, Morton P L and Sands M 1973 "Evaluation of synchrotron radiation integrals" IEEE Trans. Nucl. Sci. 20 pp 900-901 
[21] Guignard G 1978 "A General treatment of resonances in accelerators" CE R N Y ellow Report $78-11$

[22] Piwinski A 1980 "Synchro-Betatron resonances" 11th Int. Conf. on High-energy Accelerators (Geneva) pp 638-649

[23] Chao A W 1993 Physics of collective beam instabilities in high energy accelerators (John Wiley \& Sons) first edition

[24] Kohaupt R D 1979 "Single beam instabilities in PETRA" IEEE Trans. Nucl. Sci. NS 26 p 3480

[25] Kohaupt R D 1980 "Simplified presentation of head tail turbulence" DE SY Report M 80-19

[26] Talman R 1982 Nucl. Instrum. Methods 193423

[27] Seeman J T 1985 "Observations of the beam-beam interaction" Lecture Notes in P hysics 247 ed J. M. Jowett et al. (Springer-Verlag) pp 121-153.

[28] Myers S 1985 "Review of beam-beam simulations" Lecture Notes in Physics 247 ed J. M. Jowett et al. (Springer-Verlag) pp 176-237

[29] Burkhardt H 1997 "Energy dependence of beam-beam interactions at LEP" P roc. of the 17th Particle Accelerator Conf. (Vancouver) vol. 2 ed M. Comyn et al. (New York: IEEE) pp $1532-1536$

[30] Burkhardt H 1998 "Beam-beam interaction and beam lifetime in LEP" P roc. of the 14th Advanced ICFA Beam Dynamics Workshop on Beam Dynamics I ssues for $\mathrm{e}^{+} \mathrm{e}^{-}$Colliders ed L Palumbo and G Vignola (Frascati physics series 10) pp 327 - 332

[31] Brandt D et al 1998 "A candidate low emittance lattice for LEP at its highest energies" Proc. of the 6th E uropean Particle Accelerator Conf. (Stockholm) ed S. Myers et al. (Bristol: IOP) pp 394-396

[32] Amman F and Ritson D 1961 "Space-charge effects in electron-electron and positronelectron colliding or crossing beam rings" P roc. of the 1961 High E nergy Accelerator Conf. (B rookhaven National Lab.) p 471-475

[33] Amman F 1971 "Electron and positron storage rings: present situation and future prospects" Proc. of the 1971 High Energy Accelerator Conference (Geneva) pp 63-71

[34] Reichel I 1998 "Studies of the Transverse Beam Profile at LEP" CERN Thesis CERNTHESIS-98-017

[35] Burkhardt H, Reichel I and Roy G 1999 "Transverse beam tails due to inelastic scattering" CER N Report CERN-SL-99-068 (OP)

[36] Burkhardt H and Kleiss R 1994 "Beam lifetimes in LEP" P roc. of the 4th E uropean Particle Accelerator Conf. (London) vol. II ed V. Suller and C. Petit-Jean-Genaz (World Sci.) pp 1353-1355

[37] Tsai Y S 1974 Rev. Mod. Phys. 46 815-851

[38] Telnov V I 1987 Nucl. Instrum. Methods A 260304

[39] Dehning B et al. 1990 Phys. Lett. B249 145-148

[40] Altarelli G and Bucella F 1964 Nouvo Cimento 341337

[41] Altarelli G and Stella B 1974 Nuovo Cimento Lett. 9416

[42] Kleiss R and Burkhardt H 1994 Comput. Phys. Commun. 81 372-380

[43] Ball A H et al 1998 Nucl. Instrum. Methods A 403 205-246 
[44] Batterman and Bilderback 1991 Handbook on Synchrotron Radiation Vol.3 ed G S Brown and D E Moncton (Amsterdam: North Holland) pp 120-124

[45] von Holtey G, Niessen W and Roudeau P 1994 "Synchrotron radiation masks for LEP2" Proc. of the 4th European Particle Accelerator Conf. (London) ed V Suller and C PetitJean-Genaz (World Sci.) pp 454-456

[46] Lepeule P, Menot C and Veness R 1996 "Design and implementation of synchrotron radiation masks for LEP2" P roc. of the 5th European Particle Accelerator Conf. (Sitges) ed S Myers et al. (Bristol: IOP) pp 2503-2505

[47] Böge M et al. 1996 "Measurements of collision offsets and difference in vertical dispersion at the LEP interaction points" P roc. of the 5th European Particle Accelerator Conf. (Sitges) ed S Myers et al. (Bristol: IOP) pp 427-429

[48] Bovet C et al. 1996 "Luminosity optimisation using beam-beam deflections at LEP" Proc. of the 5th European Particle Accelerator Conf. (Sitges) ed S Myers et al. (Bristol: IOP) pp 325-327

[49] Lamont M 1999 "LEP : review of 1998" Proc. of the 9th W orkshop on LEP-SPS Performance (Chamonix) ed J Poole CERN Report CERN-SL-99-007 (DI) p 163

[50] Assmann R et al. 1999 "Dispersion free IPs" Proc. of the 9th Workshop on LEP-SPS Performance (Chamonix) ed J Poole CER N Report CERN-SL-99-007 (DI) p 266

[51] Press W, Flannery B, Teukolsky S and Vetterling W 1987 Numerical Recipes (Cambridge University Press)

[52] Morpurgo G 1999 "Do we understand the luminosity imbalance ?" Proc. of the 9th W orkshop on LEP-SPS Performance (Chamonix) ed J Poole CER N Report CERN-SL-99-007 (DI) p 256

[53] Sokolov A A and Ternov I M 1964 Sov. Phys. Dokl. 81203

[54] Bargmann V, Michel L and Telegdi V L 1959 Phys. Rev. Lett. 2435

[55] Arnaudon L el al. 1994 "Recent achievements in transverse spin-polarisation at LEP" Proc. of the 4th European Particle Accelerator Conf. (London) ed V Suller and C Petit-JeanGenaz (World Sci.) pp 187-189

[56] Assmann R et al 1994 "Deterministic harmonic spin matching in LEP" Proc. of the 4th E uropean Particle Accelerator Conf. (London) ed V Suller and C Petit-Jean-Genaz (World Sci.) pp 932-934

[57] Assmann R et al 1999 "Observation of radiative spin-polarization at $60.6 \mathrm{GeV}$ " P roc. of the 1999 Particle Accelerator Conf. (New York) vol. 5 ed A. Luccio and W MacKay (New York: IEEE) pp 2999-3001

[58] Arnaudon L et al. 1993 Phys. Lett. B307 187

[59] Arnaudon L et al. 1995 Z. Phys. C 66 45-62

[60] Baier V N and Orlov Y F 1966 Sov. Phys. Dokl. 101145

Zholentz A A et al. 1980 Phys. Lett. B96 214

Artamonov A S et al. 1982 Phys. Lett. B118 225

Shatunov Y M and Skrinsky A N 1989 Particle W orld Vol. 1 No. 2 p 35

Barber D P et al. 1984 P hys. Lett. B135 498

Mckay W W et al. 1984 Phys. Rev. D 292483

[61] Assmann R et al. 1995 Z. Phys. C 66 567-582 
[62] Assmann R et al. 1999 Eur. Phys. J . C 6 187-223

[63] Bravin E et al. 1998 Nucl. Instrum. Methods A 417 9-15

[64] Arnaudon L et al. 1995 Nucl. Instrum. Methods A 357 249-252

[65] Wenninger J 1999 "Observation of the radial ring deformations using closed orbits at LEP" P roc. of the $1999 \mathrm{Particle}$ Accelerator Conf. (N ew Y ork) vol. 5 ed A. Luccio and W MacKay (New York: IEEE) pp 3014-3016

[66] Bassetti M and Jowett J M 1987 "Improving the energy resolution of LEP experiments" Proc. of the 1987 Particle Accelerator Conf. (Washington, DC) (New York: IEEE) pp 115117

[67] Blondel A et al. 1999 Eur. Phys. J . C 11 573-585

[68] Burkhardt H, Muller A S and Wenninger J 1999 "Energy loss measurements at LEP2" P roc. of the $1999 \mathrm{P}$ article Accelerator Conf. ( N ew Y ork) vol. 5 ed A. Luccio and W MacKay (New York: IEEE) pp 3011-3013

[69] Janot P 2000 "Priorities for LEP in 2000" Proc. of the 10th Workshop on LEP-SPS Performance (Chamonix) ed J Poole CER N Report CERN-SL-2000-007 (DI) p 250 